KUL-TF-95/16

ULB-TH-95/07

hep-th/9505173

\title{
Local BRST cohomology in Einstein-Yang-Mills theory
}

\author{
Glenn Barnich ${ }^{1, *}$, Friedemann Brandt ${ }^{2, \dagger}$ \\ and Marc Henneaux ${ }^{1, \text { 甫 }}$
}

1 Faculté des Sciences, Université Libre de Bruxelles, Campus Plaine C.P. 231, B-1050 Bruxelles, Belgium

${ }^{2}$ Instituut voor Theoretische Fysica, Katholieke Universiteit Leuven, Celestijnenlaan 200 D, B-3001 Leuven, Belgium

*Aspirant au Fonds National de la Recherche Scientifique (Belgium).

E-mail address: gbarnich@ulb.ac.be

${ }^{\dagger}$ Supported by the research council (DOC) of the K.U. Leuven.

E-mail address: Friedemann.Brandt@fys.kuleuven.ac.be

¥Also at Centro de Estudios Científicos de Santiago, Chile.

E-mail address: henneaux@ulb.ac.be 


\begin{abstract}
We analyse in detail the local BRST cohomology in Einstein-YangMills theory using the antifield formalism. We do not restrict the Lagrangian to be the sum of the standard Hilbert and Yang-Mills Lagrangians, but allow for more general diffeomorphism and gauge invariant actions. The analysis is carried out in all spacetime dimensions larger than 2 and for all ghost numbers. This covers the classification of all candidate anomalies, of all consistent deformations of the action, as well as the computation of the (equivariant) characteristic cohomology, i.e. the cohomology of the spacetime exterior derivative in the space of (gauge invariant) local differential forms modulo forms that vanish on-shell. We show in particular that for a semi-simple Yang-Mills gauge group the antifield dependence can be entirely removed both from the consistent deformations of the Lagrangian and from the candidate anomalies. Thus, the allowed deformations of the action necessarily preserve the gauge structure, while the only candidate anomalies are those provided by previous works not dealing with antifields, and by "topological" candidate anomalies which are present only in special spacetime dimensions $(6,9,10,13, \ldots)$. This result no longer holds in presence of abelian factors where new candidate anomalies and deformations of the action can be constructed out of the conserved Noether currents (if any). The Noether currents themselves are shown to be covariantizable, i.e. they can be chosen to be invariant under local Lorentz and Yang-Mills transformations and covariant under diffeomorphisms, with a few exceptions discussed as well.
\end{abstract}




\section{Introduction}

\subsection{Motivation}

This paper deals with the following physical questions that arise in $n$-dimensional Einstein-Yang-Mills theory (as they actually arise in any local field theory).

(i) Characteristic cohomology [1]:

Do there exist non-trivial local spacetime $p$-forms constructed out of the fields and their derivatives that are conserved, i.e. closed modulo the equations of motion? A conserved $p$-form is said to be trivial if it is weakly exact, i.e. exact on-shell. In particular, what is the structure of the conserved local currents $(p=n-1)$ ? Are there nontrivial conserved $(n-2)$-forms ("charge without charge" in Wheeler's terminology [2, 3])? Of particular interest are these questions in the restricted space of gauge invariant local forms ("equivariant characteristic cohomology").

(ii) Consistent deformations of the action [4]:

Can one add non-trivial "interaction terms" to the action in a way that maintains the number (but not necessarily the form nor the group structure) of the gauge symmetries? Such deformations are called "consistent" and are regarded as trivial if they arise from a mere local redefinition of the fields. The consistent deformations determine the possible quantum corrections to the action (in the absence of anomalies). Wellknown solutions are given by invariant functions of the curvatures, the matter fields and their covariant derivatives. They do not change the gauge structure. It is of interest to determine whether there are other deformations.

(iii) Candidate anomalies:

What are the possible anomalies in the quantum theory, i.e. what is the structure of the possible breakings of the gauge symmetries by quantum effects?

These important questions have already received quite a lot of attention in the past, under restrictive assumptions. For instance, in [3] Unruh has shown the absence of "charges without charges" in Einstein gravity that 
would be at most of degree 5 in derivatives (of the metric) and covariant, i.e. constructable out of the undifferentiated metric, the Riemann tensor and its covariant derivatives. Similarly, the problem of consistent deformations of the Einstein theory has been treated by Wald in his analysis of possible spin-2 theories [5], but always under the assumption that the algebra of the deformed gauge symmetries still closes off-shell (under commutation). Finally, the classification of the anomalies has been discussed at length in [6, 7], where complete results on the candidate anomalies not containing the sources for the BRST variations (antifields) are given. The purpose of this paper is to re-address the issues (i)-(iii) above without making any a priori simplifying assumptions on the solutions or the spacetime dimension (except that we assume the latter to be larger than 2).

\subsection{Method}

Our approach is entirely based on the reformulation of (i)-(iii) in terms of the Wess-Zumino consistency condition when rewritten in BRST language, extended to arbitrary ghost number and form degree and taking into account the equations of motion. The Wess-Zumino consistency condition then reads

$$
s a^{g, p}+d a^{g+1, p-1}=0
$$

where $s$ is the Becchi-Rouet-Stora-Tyutin (BRST) operator [8]-[12] given explicitly below and where $d$ is the spacetime exterior derivative. The local forms $a^{i, j}$ have ghost number $i$ and form degree $j$ where $i$ takes integer values (including negative ones) and $0 \leq j \leq n$. They involve both the fields, the ghosts, the antifields and their derivatives. Trivial solutions of (1.1) are given by $a^{g, p}=s b^{g-1, p}+d b^{g, p-1}$ and satisfy indeed (1.1) due to

$$
s^{2}=s d+d s=d^{2}=0 .
$$

Equation (1.1) defines the local BRST cohomological group $H^{g, p}(s \mid d)$ as the set of equivalence classes of solutions $a^{g, p}$ of (1.1) modulo trivial ones.

In [13] Wess and Zumino showed that candidate anomalies must fulfill (1.1) for $g=1$ and $p=n$. It is easy to verify that consistent deformations of the action are solutions of (1.1) with $g=0$ and $p=n$ [4]. Perhaps less known is the fact that the characteristic cohomology is also described by the WessZumino consistency condition, but this time at negative ghost number. This 
was proved in [14]. Consequently, the issues (i)-(iii) are indeed equivalent to solving the Wess-Zumino consistency condition for values of the ghost number $\leq 1$, which provides a unified approach to these questions.

\subsection{Main results}

We indicate in this paper how to compute the BRST cohomology groups $H^{g, p}(s \mid d)$ for Einstein-Yang-Mills theory, and give the form of the most general cocycle (for all values of the ghost number and the form degree). Our main results, which were partly announced in [15], can be summarized as follows.

(i) Characteristic cohomology:

The conserved $p$-forms $(p \leq n-1)$ are of two types.

(i.a) Topological conserved $p$-forms.

These are closed (but non-exact) $p$-forms $a^{p}$ which fulfill

$$
d a^{p}=0, \quad a^{p} \neq d b^{p-1}
$$

identically, without invoking the equations of motions. Their existence (on a spacetime manifold with $\mathbf{R}^{n}$ topology) follows from the non-triviality of the vielbein manifold. They generalize the conservation laws found by Finkelstein and Misner [16]. The topological conserved $p$-forms may be chosen to involve only the vielbeins and their exterior derivatives and can be described in terms of the Lie algebra cohomology of $s o(n)$.

(i.b) Dynamically conserved $p$-forms.

Conserved $p$-forms of the second type are only weakly closed,

$$
d a^{p} \approx 0, \quad a^{p} \not \approx d b^{p-1}
$$

where $\approx$ denotes weak equality, i.e. equality up to terms that vanish onshell, and it is understood that all topological conserved $p$-forms have been removed from $a^{p}$. Dynamically conserved $p$-forms can be expressed entirely in terms of objects that transform tensorially under the gauge symmetries. For $p<n-2$, there are no non-trivial dynamically conserved $p$-forms. For $p=n-2$, there is one conserved $(n-2)$-form for each "free" abelian YangMills gauge field (i.e. for each abelian factor of the gauge group under which all the matter fields are uncharged), and these forms constitute a complete 
set of inequivalent dynamically conserved $(n-2)$-forms. They correspond to the "charge without charge" mechanism of Wheeler. Finally, while the characteristic cohomology for $p \leq n-2$ is relatively insensitive to the form of the Lagrangian, this is not the case for $p=n-1$. According to Noether's first theorem [17] there may exist dynamically conserved currents if the action has global symmetries, e.g. baryon number symmetry. This depends in particular on the matter part of the action (the absence of non-trivial conserved currents for pure $n=4$ Einstein gravity has been demonstrated in 18]). Since we do not restrict our investigation to a particular action, we do not investigate this question in detail, but rather prove that all Noether currents may be chosen to be invariant under local Lorentz and Yang-Mills gauge transformations and covariant under diffeomorphisms, with a few exceptions discussed in the text.

For the equivariant characteristic cohomology we prove that its cohomology classes are represented by the covariantized non-trivial Noether currents (written as $(n-1)$-forms), by $(n-2)$-forms related with "free" abelian gauge fields and by invariant polynomials in the curvature 2-forms. In particular, the latter are shown to be non-exact (even on-shell) in the space of invariant $p$-forms for $p<n-1$. This holds also in degree $(n-1)$ (relevant only in odd dimensions) if "free" abelian gauge fields are absent, and in degree $n$ (in even dimensions) it holds in absence of non-trivial Noether currents containing Chern-Simons terms.

(ii) Consistent deformations of the action:

Ghost number zero solutions of the Wess-Zumino consistency condition independent of the antifields define deformations of the action that do not modify the gauge symmetry. Solutions linear in the antifields change the gauge symmetry, but in such a way that the algebra of the new gauge transformations still closes off-shell. Solutions quadratic (or of higher order) in the antifields modify more radically the gauge symmetry, since the algebra of the new gauge transformations ceases to close off-shell.

Our main result here is that the most general solution $a^{0, n}$ of (1.1) can always be redefined such that it does not involve the antifields, provided the Yang-Mills gauge group is semi-simple. Hence, in that case the form of the gauge transformations can be kept intact under any deformation of the action. This justifies the assumptions made by Wald, and implies that the only consistent deformations are given by scalar densities constructed 
out of the curvatures, the matter fields and their covariant derivatives and the undifferentiated vielbein (plus topological and Chern-Simons terms in appropriate dimensions).

This result does not hold however, if the Yang-Mills gauge group has abelian factors. Then new solutions involving the conserved Noether currents (if any) and the antifields exist and are discussed in the text.

(iii) Candidate anomalies:

A similar result is derived for the anomalies. Namely we show that the antifield dependence can be removed from all candidate anomalies except if abelian factors are present. Hence, for semi-simple Yang-Mills gauge groups all candidate anomalies are given by the well-known "chiral anomalies" already discussed by previous authors and by "topological candidate anomalies". The latter originate from the non-trivial De Rham cohomology of the vielbein manifold and occur in $n=n_{0}+4 k$ dimensions where $n_{0}=6,9,20,35$ $(k=0,1, \ldots)$. All the chiral anomalies remain non-trivial even on-shell unless the action contains unconventional couplings.

In presence of abelian factors and conserved Noether currents, there are further candidate anomalies which are described in the text and involve the antifields.

\subsection{Outline of the paper}

The construction of the general solution of the Wess-Zumino consistency condition (1.1) is rather lengthy and involves many technical steps. We therefore give in this subsection a general outline of our strategy with an emphasis on the main ideas. The details are developed in the text.

There are in fact two essential ingredients in the calculation of $H^{*, *}(s \mid d)$. The first one is the close relationship that exists in diffeomorphism invariant theories between $H^{*, *}(s \mid d)$ and the more standard BRST cohomological groups $H^{*}(s)$. This was shown already in [7, 19] where however the topological aspects of the vielbein manifold have not been taken into account. The cohomology groups $H^{*}(s)$ are defined as the set of equivalence classes of solutions of the BRST cocycle condition

$$
s \alpha=0
$$


modulo trivial solutions of the form $s \beta$ where $\alpha$ and $\beta$ are local functions (0forms). It turns out that the knowledge of $H^{*}(s)$ enables one to completely construct $H^{*, *}(s \mid d)$. The problem of computing the cohomology of $s$ modulo $d$ can therefore be reduced to that of computing the cohomology of $s$. This property holds because the diffeomorphism ghosts - which play somehow a role similar to the differentials $d x^{\mu}$-are included among the ghosts. The relationship between $H^{*}(s)$ and $H^{*, *}(s \mid d)$ is derived in sections 3 6 where (i) a redefinition of the dynamical variables is given that simplifies the form of $s$ and the computation of its cohomology; (ii) the cohomology of $d$ is investigated in the space of local forms - it is here that the topological currents related to the topology of the vielbein manifold appear; and (iii) the map between $H^{*, *}(s \mid d)$ and $H^{*}(s)$ defined by the descent equations is analyzed.

To find the general solution of the Wess-Zumino consistency condition (1.1), it is thus sufficient to compute $H^{*}(s)$. It is here that the second important ingredient is used, namely that $s$ is the sum of the so-called Koszul-Tate differential and the "longitudinal differential along the gauge orbits". This feature is quite standard and actually at the heart of the antifield formalism [20]. It enables us to compute $H^{*}(s)$ completely using (a) the resolution properties of the Koszul-Tate complex, (b) the known mathematical results on the Lie algebra cohomology of the Lorentz and Yang-Mills gauge groups, and (c) the equivariant characteristic cohomology which we compute separately in appendix $\mathbf{E}$ by means of the results on the cohomology of the Koszul-Tate differential modulo $d$ derived in [14. This is the hard core of an analysis carried out in section 0 .

Sections 810 are comparatively much easier and simply put together the two ingredients explained above to get the results announced in subsection 1.3. We recommend these sections to readers who are interested in the results but not in the details of the computation. 


\section{Assumptions and notation}

Spacetime manifold:

In principle, one should study the general solution of the Wess-Zumino consistency condition (1.1) for gravity coupled to Yang-Mills gauge fields that may live on non-trivial bundles. Furthermore, one should allow for general spacetime manifolds $M$. This means that in general there is no global section in the bundle over $M$ of linear frames: it is not possible to choose continuously everywhere on spacetime a set of $n$ linearly independent tangent vectors. Similarly, the Yang-Mills connections may not be globally defined (as Lie-algebra valued 1-forms over spacetime).

However, before tackling the difficulties that arise because of non-trivial global features, it appears necessary to analyse the Wess-Zumino consistency condition first on a local neighbourhood $U$ homeomorphic to $\mathbf{R}^{n}$ which trivializes the bundle, i.e. which is such that the bundle reduces, over $U$, to the trivial direct product $U \times F$ of $U$ with the "fiber" $F$ of the field-antifield manifold. One can then study how the local results can be pasted together to provide global results. In this paper, we completely solve the first step. Since the neighbourhood $U$ is homeomorphic to $\mathbf{R}^{n}$, we shall actually consider that $M$ is just $\mathbf{R}^{n}$. We carry out the analysis for all $n>2$ (our methods apply also to the case $n=2$ but one encounters some subtleties in this case, cf. [14).

Field content and gauge invariances:

We consider gravitational theories whose classical action is invariant under general coordinate transformations (diffeomorphisms), local Lorentz transformations and Yang-Mills gauge transformations. The Lorentz group is denoted by $G_{L}$, the Yang-Mills gauge group by $G_{Y M}$, their Lie algebras by $\mathcal{G}_{L}$ and $\mathcal{G}_{Y M}$ where the latter is supposed to be reductive (= semi-simple + abelian). We adopt the vielbein formulation of gravity since we allow for matter fields with integer and half-integer spins. The matter fields are denoted by $y^{m}$ and are assumed to transform scalarly under general coordinate transformations (with no loss of generality, since world indices can be converted to Lorentz indices by means of the vielbein) and linearly under $G_{Y M} \times G_{L}$. The elements of $\mathcal{G}_{Y M}$ are denoted by $\delta_{i}$, the corresponding gauge fields and ghosts by $A_{\mu}{ }^{i}$ and $C^{i}$. The elements of $\mathcal{G}_{L}$ are denoted by $l_{a b}=-l_{b a}$, the corresponding gauge fields (= components of the spin con- 
nection) and ghosts by $\omega_{\mu}^{a b}=-\omega_{\mu}^{b a}$ and $C^{a b}=-C^{b a}$. The independent elements of $\mathcal{G}:=\mathcal{G}_{Y M}+\mathcal{G}_{L}$ and the corresponding gauge fields and ghosts are labeled by $I$ :

$$
\begin{aligned}
& \left\{\delta_{I}\right\}=\left\{\delta_{i}, l_{a b}: a>b\right\}, \\
& \left\{C^{I}\right\}=\left\{C^{i}, C^{a b}: a>b\right\}, \quad\left\{A_{\mu}{ }^{I}\right\}=\left\{A_{\mu}{ }^{i}, \omega_{\mu}{ }^{a b}: a>b\right\} .
\end{aligned}
$$

The structure constants of $\mathcal{G}$ and $\mathcal{G}_{Y M}$ are denoted by $f_{I J}{ }^{K}$ and $f_{i j}{ }^{k}$ respectively:

$$
\begin{aligned}
& {\left[\delta_{I}, \delta_{J}\right]=f_{I J}{ }^{K} \delta_{K}} \\
& {\left[\delta_{i}, \delta_{j}\right]=f_{i j}{ }^{k} \delta_{k}, \quad\left[l_{a b}, l_{c d}\right]=2 \eta_{a[c} l_{d] b}-2 \eta_{b[c} l_{d] a}, \quad\left[\delta_{i}, l_{a b}\right]=0}
\end{aligned}
$$

where $\eta_{a b}$ are the entries of the Minkowski metric (cf. appendix A).

We define the spin connection and the Christoffel connection through

$$
\partial_{\mu} e_{\nu}^{a}-\omega_{\mu b}{ }^{a} e_{\nu}^{b}-\Gamma_{\mu \nu}{ }^{\rho} e_{\rho}^{a}=0, \quad \omega_{\mu}^{a b}=-\omega_{\mu}^{b a}, \quad \Gamma_{\mu \nu}{ }^{\rho}=\Gamma_{\nu \mu}{ }^{\rho}
$$

which determines $\omega_{\mu}^{a b}$ and $\Gamma_{\mu \nu}{ }^{\rho}$ in terms of the vielbein fields $e_{\mu}{ }^{a}$ and their derivatives:

$$
\begin{aligned}
& \omega_{\mu}^{a b}=E^{a \nu} E^{b \rho}\left(\omega_{[\mu \nu] \rho}-\omega_{[\nu \rho] \mu}+\omega_{[\rho \mu] \nu}\right), \quad \omega_{[\mu \nu] \rho}=e_{\rho a} \partial_{[\mu} e_{\nu]}^{a}, \\
& \Gamma_{\mu \nu}^{\rho}=\frac{1}{2} g^{\rho \sigma}\left(\partial_{\mu} g_{\nu \sigma}+\partial_{\nu} g_{\mu \sigma}-\partial_{\sigma} g_{\mu \nu}\right) .
\end{aligned}
$$

Here $E_{a}{ }^{\mu}$ are the components of the inverse vielbein and $g_{\mu \nu}, g^{\mu \nu}$ are the components of the spacetime metric and its inverse (Lorentz indices $a, b, \ldots$ are raised and lowered by means of the Minkowski metric and its inverse):

$$
E_{a}{ }^{\mu} e_{\mu}{ }^{b}=\delta_{a}^{b}, \quad e_{\mu}{ }^{a} E_{a}{ }^{\nu}=\delta_{\mu}^{\nu}, \quad g_{\mu \nu}=e_{\mu a} e_{\nu}{ }^{a}, \quad g^{\mu \nu}=E^{a \mu} E_{a}{ }^{\nu} .
$$

The set of fields is therefore given by

$$
\left\{\Phi^{A}\right\}=\left\{e_{\mu}^{a}, A_{\mu}^{i}, y^{m}, \xi^{\mu}, C^{I}\right\}
$$

where the $\xi^{\mu}$ are the ghosts of diffeomorphisms. The corresponding antifields are denoted by

$$
\left\{\Phi_{A}^{*}\right\}=\left\{e_{a}^{* \mu}, A_{i}^{* \mu}, y_{m}^{*}, \xi_{\mu}^{*}, C_{I}^{*}\right\} .
$$

Note that (2.2) and (2.3) represent no loss of generality compared with other choices of $\omega_{\mu}^{a b}$ and $\Gamma_{\mu \nu}{ }^{\rho}$, as considered e.g. in [21], since non-vanishing 
torsion components (or the fields they are built of) can be counted among the matter fields $y^{m}$.

Local functions and forms:

We regard the $\partial_{\mu_{1} \ldots \mu_{k}} \Phi^{A}$ and $\partial_{\mu_{1} \ldots \mu_{k}} \Phi_{A}^{*}(k=0,1, \ldots)$ as local coordinates of a jet space $J^{\infty}$, cf. [14 and references given there. Throughout this paper a local function is by definition a function of these generators depending polynomially on all of them except on the undifferentiated $e_{\mu}{ }^{a}$ on which it must depend smoothly and regularly for $\operatorname{det}\left(e_{\mu}{ }^{a}\right) \neq 0$. The algebra of local functions is denoted by $\mathcal{A}$. A local form is by definition a finite linear combination of local functions $\alpha_{\tau}$ with coefficients $\omega^{\tau}(x, d x)$ which are differential forms on the spacetime manifold $M$ and whose algebra is denoted by $\Omega(M)$. The algebra of local forms is denoted by $\mathcal{E}$. We thus have

$$
\begin{aligned}
& \mathcal{E}=\Omega(M) \otimes \mathcal{A} ; \\
& a \in \mathcal{E} \Leftrightarrow a=\sum_{\tau} \omega^{\tau} \alpha_{\tau}, \quad \omega^{\tau} \in \Omega(M), \alpha_{\tau} \in \mathcal{A}
\end{aligned}
$$

where finiteness of the sum is understood. Note that the explicit dependence on the coordinates and the form degree of a local $p$-form are carried exclusively by $p$-forms $\omega^{\tau} \in \Omega(M)$ whereas its dependence on the fields, antifields and their derivatives and its ghost number is carried by local functions $\alpha_{\tau} \in \mathcal{A}$.

Action functional and BRST operator:

We assume that the classical action

$$
S_{0}=\int d^{n} x \mathcal{L}_{0}\left(\phi, \partial \phi, \ldots, \partial^{k} \phi\right), \quad \phi \equiv\left(e_{\mu}{ }^{a}, y^{m}, A_{\mu}{ }^{i}\right)
$$

is an integrated local function $\mathcal{L}_{0}$ and does not possess any non-trivial gauge invariances apart from those mentioned above (invariance under diffeomorphisms and gauge invariance under $\left.G_{L} \times G_{Y M}\right)$. Hence, we exclude for instance supergravity from the discussion. Then the proper solution $\mathcal{S}$ of the classical master equation [12] is given by

$$
\begin{aligned}
& \mathcal{S}=\int d^{n} x\left(\mathcal{L}_{0}-\xi^{\mu} \Phi_{A}^{*} \partial_{\mu} \Phi^{A}-C^{I} y_{m}^{*} \delta_{I} y^{m}+e_{a}^{* \mu}\left(e_{\nu}{ }^{a} \partial_{\mu} \xi^{\nu}+e_{\mu}{ }^{b} C_{b}{ }^{a}\right)\right. \\
&\left.+A_{i}^{* \mu}\left(\partial_{\mu} C^{i}+C^{j} A_{\mu}{ }^{k} f_{k j}{ }^{i}+A_{\nu}{ }^{i} \partial_{\mu} \xi^{\nu}\right)+\frac{1}{2} C_{K}^{*} C^{I} C^{J} f_{I J}{ }^{K}\right)
\end{aligned}
$$


Using the conventions of appendix A, the BRST transformations of the fields and antifields are obtained from $\mathcal{S}$ through

$$
s \Phi^{A}(x)=-\frac{\delta^{R} \mathcal{S}}{\delta \Phi_{A}^{*}(x)}, \quad s \Phi_{A}^{*}(x)=\frac{\delta^{R} \mathcal{S}}{\delta \Phi^{A}(x)}
$$

where $\delta^{R} / \delta Z(x)$ denotes the functional right derivative with respect to $Z$. The BRST transformations of derivatives of the $\Phi^{A}$ and $\Phi_{A}^{*}$ and of local functions and forms are then obtained from (2.7) using the rules

$$
s \partial_{\mu}-\partial_{\mu} s=0, \quad s(X Y)=(s X) Y+(-)^{\varepsilon(X)} X(s Y)
$$

where $\varepsilon(X)$ is the grading of $X$. The latter is defined through

$$
\begin{aligned}
& \varepsilon\left(e_{\mu}{ }^{a}\right)=\varepsilon\left(A_{\mu}{ }^{i}\right)=\varepsilon\left(y_{B}^{m}\right)=\varepsilon\left(x^{\mu}\right)=0, \\
& \varepsilon\left(y_{F}^{m}\right)=\varepsilon\left(\xi^{\mu}\right)=\varepsilon\left(C^{I}\right)=\varepsilon\left(d x^{\mu}\right)=1, \\
& \varepsilon\left(\Phi_{A}^{*}\right)=\varepsilon\left(\Phi^{A}\right)+1 \quad(\text { modulo } 2), \\
& \varepsilon\left(\partial_{\mu_{1} \ldots \mu_{r}} Z\right)=\varepsilon(Z)
\end{aligned}
$$

where the $y_{B}^{m}\left(y_{F}^{m}\right)$ are the bosonic (fermionic) $y$ 's. The differentials and coordinates have vanishing BRST transformation,

$$
s x^{\mu}=s d x^{\mu}=0 .
$$

We shall carry out the analysis without assuming a particular form of the classical action $S_{0}$, but we require that it defines a normal theory in the terminology of [14], apart from the above-mentioned assumptions on the field content, gauge invariances and locality. In order to use the definition of [14] one has to split the vielbein into a constant background vielbein and a deviation $h_{\mu}^{a}$ from it, and linearize the action resp. the field equations in the $h_{\mu}^{a}$. We stress however that we only need this split for defining the normality condition, but not as a prerequisite for the computation. In fact the normality of the theory is a sufficient condition for the validity of our results, not a necessary one, i.e. our results may apply even to non-normal theories. We do not repeat the definition of normal theories here, but we remark that most theories of physical interest are normal. An important example of a normal theory, which we will use occasionally to illustrate the general results, is provided by the usual Einstein-Yang-Mills-matter Lagrangian

$$
\mathcal{L}_{0} / e=\frac{1}{2} R-\frac{1}{4} g^{\mu \rho} g^{\nu \sigma} F_{\mu \nu}{ }^{i} F_{\rho \sigma i}+L^{y}\left(y^{m}, D_{a} y^{m}\right)
$$


where $L^{y}\left(y^{m}, D_{a} y^{m}\right)$ is at most linear (quadratic) in the $D_{a} y_{F}^{m}\left(D_{a} y_{B}^{m}\right), e$ is the determinant of the vielbein, $R=R_{\mu \nu}{ }^{a b} E_{a}{ }^{\mu} E_{b}{ }^{\nu}$ denotes the Riemann curvature scalar, $F_{\mu \nu}{ }^{i}$ are the Yang-Mills field strengthst and $D_{a} y^{m}$ are the covariant derivatives of the matter fields:

$$
\begin{aligned}
e & =\operatorname{det}\left(e_{\mu}{ }^{a}\right), \\
R_{\mu \nu}{ }^{a b} & =\partial_{\mu} \omega_{\nu}^{a b}-\partial_{\nu} \omega_{\mu}^{a b}+\omega_{\mu}^{a c} \omega_{\nu c}^{b}-\omega_{\nu}{ }^{a c} \omega_{\mu c}{ }^{b} \\
& =-E^{a \rho} e_{\sigma}{ }^{b}\left(\partial_{\mu} \Gamma_{\nu \rho}{ }^{\sigma}-\partial_{\nu} \Gamma_{\mu \rho}{ }^{\sigma}+\Gamma_{\mu \lambda}{ }^{\sigma} \Gamma_{\nu \rho}{ }^{\lambda}-\Gamma_{\nu \lambda}{ }^{\sigma} \Gamma_{\mu \rho}{ }^{\lambda}\right), \\
F_{\mu \nu}{ }^{i} & =\partial_{\mu} A_{\nu}{ }^{i}-\partial_{\nu} A_{\mu}{ }^{i}+f_{j k}{ }^{i} A_{\mu}{ }^{j} A_{\nu}{ }^{k}, \\
D_{a} y^{m} & =E_{a}{ }^{\mu}\left(\partial_{\mu}-A_{\mu}{ }^{I} \delta_{I}\right) y^{m} .
\end{aligned}
$$

\section{$3 \quad$ New basis of generators of $\mathcal{A}$}

Our fundamental variables are the fields and antifields and their partial derivatives which according to section 2 are considered as independent generators of the algebra of local functions or, in more mathematical terms, as local coordinates of a jet space $J^{\infty}$ (together with the $x^{\mu}$ ). Following the lines of [19], we shall however carry out the analysis in terms of other generators which isolates a manifestly contractible part of the algebra. The new generators serve as local coordinates of $J^{\infty}$ as well, together with the $x^{\mu}$.

The elements of the new basis that replace the fields $\Phi^{A}$ and their partial derivatives can be grouped in four sets:

$$
\begin{aligned}
& \left\{\mathcal{T}^{r}\right\}=\left\{D_{\left(a_{1} \ldots D_{\left.a_{k}\right)} y^{m}, D_{\left(a_{1}\right.} \ldots D_{a_{k}} F_{a) b}{ }^{I}: k=0,1, \ldots\right\},} \hat{\xi}^{a}=\xi^{\mu} e_{\mu}^{a}, \quad \hat{C}^{I}=C^{I}+\xi^{\mu} A_{\mu}{ }^{I},\right. \\
& \left\{U_{l}\right\}=\left\{\partial_{\left(\mu_{1} \ldots \mu_{k}\right.} e_{\mu)}^{a}, \partial_{\left(\mu_{1} \ldots \mu_{k}\right.} A_{\mu)}{ }^{I}: k=0,1, \ldots\right\} \\
& \left\{V_{l}\right\}=\left\{\partial_{\left(\mu_{1} \ldots \mu_{k}\right.} s e_{\mu)}{ }^{a}, \partial_{\left(\mu_{1} \ldots \mu_{k}\right.} s A_{\mu)}{ }^{I}: k=0,1, \ldots\right\}
\end{aligned}
$$

where

$$
F_{a b}{ }^{i}=E_{a}{ }^{\mu} E_{b}{ }^{\nu} F_{\mu \nu}{ }^{i}, \quad F_{a b}{ }^{c d}=E_{a}{ }^{\mu} E_{b}{ }^{\nu} R_{\mu \nu}{ }^{c d}, \quad D_{a}=E_{a}{ }^{\mu}\left(\partial_{\mu}-A_{\mu}{ }^{I} \delta_{I}\right)
$$

and it is understood that only algebraically independent curvature components $F_{a b}{ }^{I}$ enter in (3.1) (together with the symmetrization of the indices in

\footnotetext{
${ }^{1}$ Indices $i, j, \ldots$ referring to the semi-simple part of $\mathcal{G}_{Y M}$ are raised and lowered by means of its Cartan-Killing metric; indices $i, j, \ldots$ referring to abelian elements of $\mathcal{G}_{Y M}$ are raised and lowered by means of the unit matrix.
} 
(3.1) this guarantees the absence of algebraic identities between the generators (3.1), taking into account $\left[D_{a}, D_{b}\right]=-F_{a b}{ }^{I} \delta_{I}$ and the resulting Bianchi identities). One easily verifies that indeed each local function of the $\partial_{\mu_{1} \ldots \mu_{k}} \Phi^{A}$ can be expressed as a local function of the generators (3.1)-(3.4) and vice versal2. Skipping the details we just note that (a) the change of generators $\hat{\xi}^{a}, \hat{C}^{I} \leftrightarrow \xi^{\mu}, C^{I}$ (given the $U_{l}$ ) is invertible; (b) the derivatives of the ghosts can be replaced by the $V_{l}$ due to $s e_{\mu}{ }^{a}=\partial_{\mu} \hat{\xi}^{a}+\ldots, s A_{\mu}{ }^{I}=\partial_{\mu} C^{I}+\ldots ;$ (c) the $D_{\left(a_{1} \ldots D_{a_{k}}\right)} y^{m}$ are equivalent to the $\partial_{\mu_{1} \ldots \mu_{k}} y^{m} ;(\mathrm{d})$ the $\omega_{\mu}^{a b}$ and $\partial_{(\mu} e_{\nu)}{ }^{a}$ are equivalent to the $\partial_{\mu} e_{\nu}{ }^{a}$, cf. (2.2); (e) the $\partial_{\left(\mu_{1} \ldots \mu_{k+2}\right.} e_{\mu)}{ }^{a}, \partial_{\left(\mu_{1} \ldots \mu_{k+1}\right.} A_{\mu)}{ }^{I}$ and $D_{\left(a_{1}\right.} \ldots D_{a_{k}} F_{a) b}{ }^{I}$ are equivalent to the $\partial_{\mu_{1} \ldots \mu_{k+2}} e_{\mu}{ }^{a}$ and $\partial_{\mu_{1} \ldots \mu_{k+1}} A_{\mu}{ }^{I}$ (for each $k \geq 0$ separately).

The new generators that replace the undifferentiated antifields are

$$
\begin{aligned}
& \hat{y}_{m}^{*}=y_{m}^{*} / e, \quad \hat{e}_{a}^{* b}=e_{\mu}{ }^{b} e_{a}^{* \mu} / e, \quad \hat{A}_{i}^{* a}=e_{\mu}{ }^{a} A_{i}^{* \mu} / e, \\
& \hat{C}_{I}^{*}=C_{I}^{*} / e, \quad \hat{\xi}_{a}^{*}=E_{a}{ }^{\mu}\left(\xi_{\mu}^{*}-A_{\mu}{ }^{I} C_{I}^{*}\right) / e .
\end{aligned}
$$

Apart from the more involved form of $\hat{\xi}_{a}^{*}$ these definitions just convert the original antifields to generators transforming scalarly under general coordinate transformations [the factor $1 / e$ occurs because all antifields transform as scalar or vector densities under general coordinate transformations as follows from (2.6) and (2.7)]. The new generators that replace the partial derivatives of the $\Phi_{A}^{*}$ are the corresponding symmetrized covariant derivatives of the generators (3.6):

$$
\left\{\mathcal{T}_{\bar{r}}^{*}\right\}=\left\{D_{\left(a_{1}\right.} \ldots D_{\left.a_{k}\right)} \hat{\Phi}_{A}^{*}: k=0,1 \ldots\right\}, \quad\left\{\hat{\Phi}_{A}^{*}\right\}=\left\{\hat{y}_{m}^{*}, \hat{e}_{a}^{* b}, \hat{A}_{i}^{* a}, \hat{C}_{I}^{*}, \hat{\xi}_{a}^{*}\right\} .
$$

As mentioned above we shall compute the BRST cohomology using the new generators. We shall therefore need their BRST transformations. Those of the $U_{l}$ and $V_{l}$ are extremely simple in the new basis since they just read

$$
s U_{l}=V_{l}, \quad s V_{l}=0 .
$$

The BRST transformations of $\hat{\xi}, \hat{C}, \mathcal{T}$ and $\mathcal{T}^{*}$ are more complicated. For later purpose we decompose the BRST operator according to

$$
s=\delta+\gamma
$$

\footnotetext{
${ }^{2}$ A local function of the generators $3.1-(3.4)$ depends polynomially on all of them apart from $e_{\mu}{ }^{a}$.
} 
where $\delta$ is the Koszul-Tate differential which has been discussed and analyzed in the antifield context first in [20]. As we shall see it plays a crucial role in the subsequent calculations. What distinguishes $\delta$ and $\gamma$ is the antighost number (antigh) defined through

$$
\begin{aligned}
& \operatorname{antigh}\left(\Phi^{A}\right)=0, \\
& \operatorname{antigh}\left(y_{m}^{*}\right)=\operatorname{antigh}\left(e_{a}^{* \mu}\right)=\operatorname{antigh}\left(A_{i}^{* \mu}\right)=1, \\
& \operatorname{antigh}\left(\xi_{\mu}^{*}\right)=\operatorname{antigh}\left(C_{I}^{*}\right)=2 .
\end{aligned}
$$

The ghost number $(g h)$ is related to the antighost number by

$$
g h=\text { puregh }- \text { antigh }
$$

where puregh is defined through

$$
\begin{aligned}
& \operatorname{puregh}\left(\xi^{\mu}\right)=\operatorname{puregh}\left(C^{I}\right)=1, \\
& \operatorname{puregh}\left(y^{m}\right)=\operatorname{puregh}\left(A_{\mu}{ }^{i}\right)=\operatorname{puregh}\left(e_{\mu}{ }^{a}\right)=\operatorname{puregh}\left(\Phi_{A}^{*}\right)=0 .
\end{aligned}
$$

The differential $\delta$ is characterized by antigh $(\delta)=-1$, i.e. it lowers the antighost number by one unit and acts on $\hat{\xi}^{a}, \hat{C}^{I}, \mathcal{T}^{r}, \mathcal{T}_{\bar{r}}^{*}$ according to

$$
\begin{aligned}
& \delta \mathcal{T}^{r}=\delta \hat{C}^{I}=\delta \hat{\xi}^{a}=0, \\
& \delta \hat{y}_{m}^{*}=\frac{1}{e} \frac{\delta \mathcal{L}_{0}}{\delta y^{m}}, \quad \delta \hat{A}_{i}^{* a}=\frac{1}{e} e_{\mu}{ }^{a} \frac{\delta \mathcal{L}_{0}}{\delta A_{\mu}{ }^{i}}, \quad \delta \hat{e}_{a}^{* b}=\frac{1}{e} e_{\mu}{ }^{b} \frac{\delta \mathcal{L}_{0}}{\delta e_{\mu}{ }^{a}}, \\
& \delta \hat{C}_{i}^{*}=-D_{a} \hat{A}_{i}^{* a}+\hat{y}_{m}^{*} \delta_{i} y^{m}, \quad \delta \hat{C}_{a b}^{*}=-2 \hat{e}_{[a b]}^{*}+\hat{y}_{m}^{*} l_{a b} y^{m}, \\
& \delta \hat{\xi}_{a}^{*}=-D_{b} \hat{e}_{a}^{* b}-\hat{A}_{i}^{* b} F_{b a}{ }^{i}+\hat{y}_{m}^{*} D_{a} y^{m}, \\
& \delta D_{a_{1}} \ldots D_{a_{k}} \hat{\Phi}_{A}^{*}=D_{a_{1}} \ldots D_{a_{k}} \delta \hat{\Phi}_{A}^{*}
\end{aligned}
$$

where $\delta \mathcal{L}_{0} / \delta \phi$ denotes the Euler-Lagrange right-derivative of $\mathcal{L}_{0}$ with respect to $\phi$,

$$
\frac{\delta \mathcal{L}_{0}}{\delta \phi}=\sum_{k \geq 0}(-)^{k} \partial_{\mu_{1}} \ldots \partial_{\mu_{k}} \frac{\partial^{R} \mathcal{L}_{0}}{\partial\left(\partial_{\mu_{1} \ldots \mu_{k}} \phi\right)} .
$$

We remark that $\delta \hat{y}_{m}^{*}, \delta \hat{A}_{i}^{* a}$ and $\delta \hat{e}_{a}^{* b}$ can be always expressed entirely in terms of the $\mathcal{T}^{r}$, independently of the particular choice of $\mathcal{L}_{0}$, since the equations of motion are necessarily covariant (in fact this can be easily proved using 
the decomposition $\delta+\gamma$ of $s$ and its nilpotency). For instance, (2.11) yields

$$
\begin{array}{lll}
\delta \hat{A}_{i}^{* a} \stackrel{(2.11)}{=} & D_{b} F^{b a}{ }_{i}+\frac{1}{e} e_{\mu}{ }^{a} \frac{\delta\left(e L^{y}\right)}{\delta A_{\mu}{ }^{i}}, \\
\delta \hat{e}_{a}^{* b} \stackrel{(2.11)}{=} & -R_{a}{ }^{b}+\frac{1}{2} \delta_{a}^{b} R+F_{a c}{ }^{i} F^{b c}{ }_{i}-\frac{1}{4} \delta_{a}^{b} F_{d c}{ }^{i} F^{d c}{ }_{i} \\
& & +\frac{1}{e} e_{\mu}{ }^{b} \frac{\delta\left(e L^{y}\right)}{\delta e_{\mu}{ }^{a}}
\end{array}
$$

where $R_{a b}=R_{a c b}^{c}$ is the Ricci tensor.

The differential $\gamma$ is characterized by antigh $(\gamma)=0$ and acts on $\hat{\xi}^{a}, \hat{C}^{I}$, $\mathcal{T}^{r}, \mathcal{T}_{\bar{r}}^{*}$ according to

$$
\begin{aligned}
& \gamma \mathcal{T}^{r}=\left(\hat{\xi}^{a} D_{a}+\hat{C}^{I} \delta_{I}\right) \mathcal{T}^{r}, \quad \gamma \mathcal{T}_{\bar{r}}^{*}=\left(\hat{\xi}^{a} D_{a}+\hat{C}^{I} \delta_{I}\right) \mathcal{T}_{\bar{r}}^{*}, \\
& \gamma \hat{\xi}^{a}=\hat{C}_{b}{ }^{a} \hat{\xi}^{b}, \quad \gamma \hat{C}^{I}=\frac{1}{2} \hat{C}^{J} \hat{C}^{K} f_{K J}{ }^{I}+\hat{F}^{I}
\end{aligned}
$$

where

$$
\hat{F}^{i}=\frac{1}{2} \hat{\xi}^{a} \hat{\xi}^{b} F_{a b}^{i}, \quad \hat{F}^{a b}=\frac{1}{2} \hat{\xi}^{c} \hat{\xi}^{d} R_{c d}^{a b} .
$$

The nilpotency of $s$ and the different antighost numbers of $\delta$ and $\gamma$ imply

$$
\delta^{2}=\gamma^{2}=\delta \gamma+\gamma \delta=0 .
$$

Using the new generators, the algebra of local functions can be written as the tensor product of two algebras,

$$
\mathcal{A}=\mathcal{A}_{1} \otimes \mathcal{A}_{2}
$$

where $\mathcal{A}_{1}$ is generated by the $U_{l}$ and the $V_{l}$, and $\mathcal{A}_{2}$ is the algebra generated by the tensor fields $\mathcal{T}^{r}, \mathcal{T}_{\bar{r}}^{*}$ and the undifferentiated ghosts $\hat{\xi}^{a}$ and $\hat{C}^{I}$. Note that, by definition of $\mathcal{A}, \mathcal{A}_{2}$ is a polynomial algebra whereas $\mathcal{A}_{1}$ is polynomial in all generators $U_{l}$ and $V_{l}$ but the undifferentiated vielbeins. The usefulness of the new basis of generators originates in the fact that both $\mathcal{A}_{1}$ and $\mathcal{A}_{2}$ are $s$-invariant subalgebras of $\mathcal{A}$, i.e. the image of $\mathcal{A}_{i}$ under $s$ is contained in $\mathcal{A}_{i}$ :

$$
s\left[\mathcal{A}_{1}\right] \subset \mathcal{A}_{1}, \quad s\left[\mathcal{A}_{2}\right] \subset \mathcal{A}_{2} .
$$

This is evident from (3.8), (3.13) and (3.16), and allows one to apply the Künneth formula for the computation of the BRST cohomology in the ghost number $g$ section of $\mathcal{A}$, denoted by $H^{g}(s, \mathcal{A})$ :

$$
s \alpha^{g}=0, \quad \alpha^{g} \in \mathcal{A} \quad \Leftrightarrow \quad \alpha^{g}=\sum_{k=0}^{g} \alpha_{(1)}^{k} \alpha_{(2)}^{g-k}+s \beta^{g-1}, \quad \beta^{g-1} \in \mathcal{A} .
$$


Here superscripts denote the ghost number and $\alpha_{(i)}^{k}$ denote representatives of cohomology classes of $H^{k}\left(s, \mathcal{A}_{i}\right)$ for $i=1,2$.

\section{Cohomology of $d$ in the algebra $\mathcal{E}$ of local forms}

Our first step is to characterize the cohomology of the exterior derivative operator $d$ in the algebra of local forms. As we shall see, this first step is not devoid of global subtleties because the manifold of the vielbeins carries some non-trivial cohomology. We shall encounter topological currents of a type reminiscent of the currents described in [16], which must be taken into account for a correct analysis.

We denote by $E$ the bundle $\mathbf{R}^{n} \times F$ where, as we have already mentioned, $F$ is the fiber of the fields and antifields. Any point in $E$ can be parametrized by $\left(x^{\mu}, \Phi^{A}, \Phi_{A}^{*}\right)$. These functions are all globally defined on $E$. However, they fail to provide global free coordinates of $E$ because the vielbein components $e_{\mu}{ }^{a}$ are constrained by the condition $\operatorname{det}\left(e_{\mu}{ }^{a}\right)>0$ (we assume the vielbeins to have positive orientation), and so, do not have a range of variation homeomorphic to $R^{n^{2}}$. Rather, the vielbeins belong to $G L^{+}(n)$, the set of matrices with strictly positive determinant. Of course the range of values which the matter fields can take may be restricted too, but for simplicity we assume that the manifold of the vielbeins is the only non-trivial factor of $E$, so that $E$ can be contracted to it. Thus, effectively one has $H_{D R}^{*}(E)=H_{D R}^{*}\left(G L^{+}(n)\right)$ which is non-trivial. In the terminology of [16], the manifold of the vielbeins is a "non-linear" manifold.

The cohomology of the exterior derivative operator $d$ in the algebra $\mathcal{E}$ of local forms is described by a theorem which has been obtained in [22] and is discussed in 23]. It generalizes well known results derived and rederived for trivial bundles by various people to a bundle $E$ which is not cohomologically trivial (besides the above mentioned references, see [24, 25]). The theorem in question is in fact valid for bundles that need not be direct products and states explicitly the following:

Theorem 4.1 : The cohomology $H^{k}(d, \mathcal{E})$ of $d$ in the algebra of local $k$-forms is isomorphic to the De Rham cohomology of $E$ in the same degree for $k<n$,

$$
H^{k}(d, \mathcal{E}) \simeq H_{D R}^{k}(E), k<n .
$$


Furthermore, in form degree $n$, one has

$$
\frac{\{\text { variationally closed } n \text {-forms }\}}{\{d \text {-exact } n \text {-forms }\}} \simeq H_{D R}^{n}(E)
$$

where the $n$-form $\omega=\mathcal{L} d^{n} x$ is said to be "variationally closed" iff the EulerLagrange derivatives of $\mathcal{L}$ with respect to all the fields and antifields identically vanish.

We shall not prove the theorem (see original literature). We shall instead draw some of its consequences for the theory at hand.

As we have pointed out, the De Rham cohomology of $E$ is isomorphic to the De Rham cohomology of the fibers (the base is trivial) and reduces to $H_{D R}^{*}\left(G L^{+}(n)\right)$ since all factors but the vielbein one are (effectively) trivial (contractible to a point). Now, the manifold $G L^{+}(n)$ is diffeomorphic (as a smooth manifold, not as a group!) to the product manifold $N \times S O(n)$, where $N$ is the set of upper triangular matrices with strictly positive real numbers on the diagonal (Gram-Schmidt decomposition). The manifold $N$ is contractible. Thus $S O(n)$ is a deformation retract of $G L^{+}(n)$. This implies that the De Rham cohomology of $G L^{+}(n)$ is isomorphic to the De Rham cohomology of $S O(n)$,

$$
H_{D R}^{*}\left(G L^{+}(n)\right) \simeq H_{D R}^{*}(S O(n)),
$$

and can be described in terms of the Lie algebra cohomology of $s o(n)$.

Given a non-trivial closed $k$-form $\alpha\left(e_{\mu}{ }^{a}, d_{G L^{+}(n)} e_{\mu}{ }^{a}\right)$ on $\left.G L^{+}(n)(k \leq n)\right)^{3}$, one gets the corresponding class $[\alpha]$ in $H^{k}(d, \mathcal{E})$ by simply replacing the exterior derivative $d_{G L^{+}(n)}$ on $G L^{+}(n)$ by the spacetime exterior derivative $d$.

We shall denote the result by $\alpha^{0, k}$ to emphasize the fact that one gets a $k$-form on $\mathcal{E}$ with ghost number 0 . Thus,

$$
\alpha^{0, k}\left(e_{\mu}^{a}, d e_{\mu}^{a}\right)=\alpha\left(e_{\mu}^{a}, d_{G L^{+}(n)} e_{\mu}^{a} \rightarrow d e_{\mu}^{a}\right)
$$

with $d e_{\mu}{ }^{a} \equiv d x^{\rho} \partial_{\rho} e_{\mu}{ }^{a}$. The Lie algebra cohomology of $s o(n)$ is finitedimensional. Thus, $H^{k}(d, \mathcal{E}) \simeq H_{D R}^{k}\left(G L^{+}(n)\right) \simeq H_{D R}^{k}(S O(n))$ is also

\footnotetext{
${ }^{3}$ At any point of $G L^{+}(n)$, the forms $d_{G L^{+}(n)} e_{\mu}{ }^{a}$ are well-defined and yield a basis of the cotangent space. Thus, any $k$-form on $G L^{+}(n)$ can be expressed as a polynomial of order $k$ in the $d_{G L^{+}(n)} e_{\mu}{ }^{a}$ with coefficients that are smooth functions of the $e_{\mu}{ }^{a}$.
} 
finite-dimensional for $k<n$. Let $\left\{\left[\alpha_{m}\right]\right\}$ be a basis of the cohomology $H_{D R}^{*}\left(G L^{+}(n)\right)$ and let $\alpha_{m}^{0, k_{m}}\left(e_{\mu}{ }^{a}, d e_{\mu}{ }^{a}\right)$ be the corresponding local forms of $\mathcal{E}$ obtained through (4.4). Let $a^{k}$ be a closed $k$-form of $\mathcal{E}$ with $k<n$. What the above theorem says is that one can express $a^{k}$ as a linear combination of the $\alpha_{m}^{0, k_{m}}\left(e_{\mu}{ }^{a}, d e_{\mu}{ }^{a}\right)$ of same degree modulo a $d$-exact form,

$$
k<n: \quad d a^{k}=0 \Leftrightarrow a^{k}=\sum_{\left\{m \mid k_{m}=k\right\}} \lambda_{m} \alpha_{m}^{0, k_{m}}+d b^{k-1},
$$

for some constants $\lambda_{m}$ and some $(k-1)$-form $b^{k-1}$. Similarly, in degree $n$, one has

$$
\frac{\delta \mathcal{L}}{\delta Z}=0 \quad \forall Z \in\left\{\Phi^{A}, \Phi_{A}^{*}\right\} \quad \Leftrightarrow \quad \mathcal{L} d^{n} x=\sum_{\left\{m \mid k_{m}=n\right\}} \lambda_{m} \alpha_{m}^{0, k_{m}}+d b^{n-1}
$$

for some constants $\lambda_{m}$ and some $(n-1)$-form $b^{n-1}$. The coefficients $\lambda_{m}$ in the decompositions (4.5) and (4.6) are unique, since the relation $\sum \lambda_{m} \alpha_{m}^{0, k_{m}}+d b=$ 0 implies $\lambda_{m}=0$.

To give an example of (4.6), consider 3-dimensional gravity. The 3-form

$$
\alpha^{0,3}=\Omega_{a}{ }^{b} \Omega_{b}{ }^{c} \Omega_{c}{ }^{a} \quad \text { with } \quad \Omega_{a}^{b}=E_{a}{ }^{\mu} d e_{\mu}{ }^{b}
$$

is easily seen to be variationally closed, but it cannot be written as the exterior derivative of a globally defined 2 -form. The integral of $\alpha^{0,3}$ is equal to the winding number of the map from the spacetime manifold to the group manifold defined by $e_{\mu}{ }^{a}$. As $\alpha^{0,3}$ above, one obtains in $n=2 r+1$ spacetime dimensions all $\alpha_{m}^{0, k_{m}}$ simply through the substitution $C_{a}{ }^{b} \rightarrow \Omega_{a}{ }^{b}$ from the corresponding ghost polynomials $P(\theta(C))$ describing the Lie algebra cohomology of $s o(n)$, cf. appendix B. Note that $\Omega^{a b}$ has both a symmetric and an antisymmetric part, contrary to $C^{a b}=-C^{b a}$. One must not omit the symmetric part of $\Omega^{a b}$ since otherwise the resulting polynomials in $\Omega^{a b}$ would not be $d$-closed. This reflects that $\alpha_{m}^{0, k_{m}}$ arises from a cohomology class of $H_{D R}\left(G L^{+}(n)\right)$ rather than of just $H_{D R}(S O(n))$. In $n=2 r$ spacetime dimensions the situation is slightly more complicated. Here one obtains all $\alpha_{m}^{0, k_{m}}$ as described above through the substitution $C_{a}{ }^{b} \rightarrow \Omega_{a}{ }^{b}$ from the $P(\theta(C))$ except for the one which corresponds to the primitive element $\theta_{r}(C)$ in the notation of appendix $\mathrm{B}\left(\theta_{r}(\Omega)\right.$ is not $d$-closed). Since $\theta_{r}(C)$ has ghost number $2 r-1=n-1$, the corresponding $\alpha_{m}^{0, k_{m}}$ is an $(n-1)$-form. It should 
therefore agree with the $(n-1)$-form discussed in [26] since further $\alpha_{m}^{0, k_{m}}$ with form-degree $(n-1)$ do not exist in all even spacetime dimensions but just for $n=4 k$ and $n=22+4 k(k=0,1, \ldots)$, and since the other $\alpha_{m}^{0, k_{m}}$ are not gauge invariant under local Lorentz rotations of the vielbein, cf. remark below.

In four dimensions, one gets non-trivial cohomology in form degrees 0 and 3, with one cohomology class in degree 0 (represented by a constant) and two cohomology classes in degree 3.

Since the cohomology is completely carried by the vielbeins, one has also the following immediate consequence of Theorem 4.1:

Corollary 4.1 : Let $a^{k}$ be a closed form of degree $k<n$ that vanishes when one sets all the fields, the antifields and their derivatives equal to zero, except the vielbeins and their derivatives. Then, $a^{k}$ is exact, $a^{k}=d b^{k-1}$. Similarly, let $a^{n}$ be a variationally closed $n$-form that vanishes under the same conditions. Then, $a^{n}$ is exact, $a^{n}=d b^{n-1}$. In particular, if $a^{k}$ is a (variationally) closed form of non-vanishing ghost number, then $a^{k}=d b^{k-1}$.

\section{Remark:}

We shall see in the next section that the forms $\alpha_{m}^{0, k_{m}}$ have counterparts $\alpha_{m}^{g, k_{m}-g}$ with non-vanishing ghost numbers $g=1, \ldots, k_{m}$, arising even for $k_{m}>n$. Depending on $n$, some of these forms contribute to solutions $a^{g, p}$ of (1.1) in physically interesting cases such as $(g, p)=(0, n)$ (possible contributions to the action) or $(g, p)=(1, n)$ (candidate anomalies). The $\alpha_{m}^{0, n}$ are somewhat analogous to the $\Theta$-term in Yang-Mills theory. They are present in $n=n_{0}+4 k$ dimensions where $n_{0}=3,10,21,36(k=0,1, \ldots)$.

The physical interpretation of the $\alpha_{m}^{0, k_{m}}$ is obscured by the fact that most of them have no counterpart in the metric formulation of gravity. Namely in the metric formulation closed and non-exact forms analogous to the $\alpha_{m}^{0, k_{m}}$ exist only in even spacetime dimensions and only in form degree $(n-1)$, if the metric has Lorentzian signature $(+,-, \ldots,-)$ [26].

Albeit the physical relevance or interpretation of the different results in the metric and the vielbein formulation of gravity remains to be uncovered, their mathematical origin is easily understood. Namely, since the metric remains unchanged under local Lorentz rotations of the vielbein $e_{\mu}{ }^{a}(x) \rightarrow$ $e_{\mu}{ }^{b}(x) \Lambda_{b}{ }^{a}(x)(\Lambda \in S O(1, n-1))$, only those linear combinations of the $\alpha_{m}^{0, k_{m}}$ can have counterparts in the metric formulation which can be cast in a form 
(by adding exact forms, if necessary) that is gauge invariant under Lorentz transformations. This invariance condition eliminates a great number of cohomological classes and leaves one with the De Rham cohomology of the (Lorentzian) metric manifold, which is two (one) dimensional in even (odd) dimensional spacetime (see e.g. [26]).

\section{$5 \quad$ BRST cohomology in $\mathcal{A}_{1}$ and Künneth for- mula for $H^{*}(s, \mathcal{E})$}

The De Rham cohomology of $G L^{+}(n)$ appears again when one analyzes the BRST cohomology in the algebra $\mathcal{E}=\Omega(M) \otimes \mathcal{A}$ of local forms. We have seen in section 3 that the algebra $\mathcal{A}$ of local functions is the direct product of two $s$-invariant subalgebras $\mathcal{A}_{1}$ and $\mathcal{A}_{2}$ generated by $\left\{U_{l}, V_{l}\right\}$ and $\left\{\mathcal{T}^{r}, \mathcal{T}_{\bar{r}}^{*}, \hat{\xi}^{a}, \hat{C}^{I}\right\}$ respectively, cf. (3.19) and (3.20). The cohomology $H^{*}\left(s, \mathcal{A}_{2}\right)$ of $s$ in $\mathcal{A}_{2}$ will be discussed at length in the sequel. We compute here $H^{*}\left(s, \mathcal{A}_{1}\right)$ and extend the Künneth formula (3.21) for $\mathcal{A}$ to $\mathcal{E}$.

Theorem 5.1 : The cohomology $H^{*}\left(s, \mathcal{A}_{1}\right)$ of $s$ in $\mathcal{A}_{1}$ is finite-dimensional and isomorphic to the De Rham cohomology of $G L^{+}(n)$,

$$
H^{*}\left(s, \mathcal{A}_{1}\right) \simeq H_{D R}^{*}\left(G L^{+}(n)\right)
$$

Proof. The cohomology of $s$ in $\mathcal{A}_{1}$ would be trivial if the vielbeins were not restricted to belong to $G L^{+}(n)$. Indeed, $s$ takes in $\mathcal{A}_{1}$ the standard contractible form due to (3.8). Therefore the generators $\left(U_{l}, V_{l}\right)$ disappear in pair whenever $U_{l}$ ranges over the entire real line. This leaves one with the single pairs $\left(e_{\mu}^{a}, s e_{\mu}^{a}\right)$, for which there is the constraint $\operatorname{det}\left(e_{\mu}{ }^{a}\right)>0$.

Now, the algebra of polynomials in $s e_{\mu}{ }^{a}$ with coefficients that are smooth functions of $e_{\mu}{ }^{a}$ (on $G L^{+}(n)$ ) is isomorphic to the exterior algebra $\Omega\left(G L^{+}(n)\right)$, and the actions of $s$ and $d_{G L^{+}(n)}$ coincide in the isomorphism. Thus $H^{*}\left(s, \mathcal{A}_{1}\right) \simeq H_{D R}^{*}\left(G L^{+}(n)\right)$, which proves the theorem.

We shall denote by $\alpha_{m}^{k_{m}, 0}\left(e_{\mu}{ }^{a}, s e_{\mu}{ }^{a}\right)$ the elements in $\mathcal{A}_{1}$ corresponding to the representatives of the classes of $H_{D R}^{*}\left(G L^{+}(n)\right)$ introduced above. These forms are simply obtained from $\alpha_{m}$ by replacing $d_{G L^{+}(n)}$ by $s$ (rather than by $d$ ). They have ghost number $k_{m}$ and form degree zero. 
For $k_{m} \leq n$ one may relate very simply $\alpha_{m}^{k_{m}, 0}\left(e_{\mu}{ }^{a}, s e_{\mu}{ }^{a}\right)$ to $\alpha_{m}^{0, k_{m}}\left(e_{\mu}{ }^{a}, d e_{\mu}{ }^{a}\right)$ through descent equations. Let

$$
\tilde{s}=s+d
$$

and let $\tilde{\alpha}_{m}=\alpha_{m}\left(e_{\mu}{ }^{a}, d_{G L^{+}(n)} e_{\mu}{ }^{a} \rightarrow \tilde{s} e_{\mu}{ }^{a}\right) . d_{G L^{+}(n)} \alpha_{m}=0$ implies $\tilde{s} \tilde{\alpha}_{m}=0$. If one expands this equation according to the ghost number, one gets a chain of "descent equations" that read explicitly

$$
\begin{gathered}
d \alpha_{m}^{0, k_{m}}=0, \\
s \alpha_{m}^{0, k_{m}}+d \alpha_{m}^{1, k_{m}-1}=0, \\
\vdots \\
s \alpha_{m}^{k_{m}-1,1}+d \alpha_{m}^{k_{m}, 0}=0, \\
s \alpha_{m}^{k_{m}, 0}=0 .
\end{gathered}
$$

Because $\alpha_{m}^{0, k_{m}}$ defines a non-trivial class in $H^{0, k_{m}}(d, \mathcal{E})$, the first of these equations does not imply that $\alpha_{m}^{0, k_{m}}$ is $d$-exact $\left(k_{m} \leq n\right)$. This is of course necessary for the consistency of the analysis, since $d$-triviality of the top form $\alpha_{m}^{0, k_{m}}$ would imply $s$-triviality of the bottom form $\alpha_{m}^{k_{m}, 0}$.

Actually, one can even prove more about $\alpha_{m}^{0, k_{m}}$. Namely, that it is a nontrivial element of $H^{0, k_{m}}(s \mid d)$ (i.e., that it remains non-trivial even on-shell). Indeed, if one had $\alpha_{m}^{0, k_{m}}=d \beta_{m}^{0, k_{m}-1}+s \beta_{m}^{-1, k_{m}}$, then one would get from $s \alpha_{m}^{0, k_{m}}+d \alpha_{m}^{1, k_{m}-1}=0$ that $\alpha_{m}^{1, k_{m}-1}-s \beta_{m}^{0, k_{m}-1}$ is $d$-closed. But since the latter has ghost number equal to one, it is exact by corollary 4.1. This implies that $\alpha_{m}^{1, k_{m}-1}$ is also trivial in $H^{1, k_{m}-1}(s \mid d), \alpha_{m}^{1, k_{m}-1}=d \beta_{m}^{1, k_{m}-2}+s \beta_{m}^{0, k_{m}-1}$. Repeating the argument would finally lead to the conclusion that the last term $\alpha_{m}^{k_{m}, 0}$ is also trivial in $H^{k_{m}}(s)$, i.e. $\alpha_{m}^{k_{m}, 0}=s \beta_{m}^{k_{m}-1,0}$ (there is no $(-1)$ form). This is the desired contradiction since $\alpha_{m}^{k_{m}, 0}$ is not $s$-exact. The same argument applies to linear combination of the $\alpha_{m}^{0, k_{m}}$ 's. Thus we have :

Theorem 5.2 : No non-vanishing linear combination of the $\alpha_{m}^{0, k_{m}}$ is trivial in $H^{*, *}(s \mid d)$,

$$
\sum_{\left\{m \mid k_{m} \leq n\right\}} \lambda_{m} \alpha_{m}^{0, k_{m}}=s b+d c \quad \Leftrightarrow \quad \lambda_{m}=0 \quad \forall m .
$$

Of course, this property is shared by all non-vanishing $\alpha^{k_{m}-p, p}$, even for $k_{m}>n$, as the same argument shows (if $k_{m}>n$ the descent equations (5.3) remain valid with $\alpha_{m}^{k_{m}-p, p} \equiv 0$ for $\left.p>n\right)$. Finally, we leave it to the reader to check that the following theorem holds: 
Theorem 5.3 : The cohomology $H^{*}(s, \mathcal{E})$ of $s$ in the algebra $\mathcal{E}=\Omega(M) \otimes$ $\mathcal{A}_{1} \otimes \mathcal{A}_{2}$ is given by $\Omega(M) \otimes H^{*}\left(s, \mathcal{A}_{1}\right) \otimes H^{*}\left(s, \mathcal{A}_{2}\right)$. That is, if a is BRST closed, sa $=0$, then

$$
a=\sum_{m, A} \omega_{m, A}(x, d x) \alpha_{m}^{k_{m}, 0}\left(e_{\mu}^{a}, s e_{\mu}^{a}\right) P_{A}\left(\mathcal{T}, \mathcal{T}^{*}, \hat{\xi}, \hat{C}\right)+s b
$$

where the polynomials $P_{A}$ define a basis of the cohomology $H^{*}\left(s, \mathcal{A}_{2}\right)$ (to be computed in section (1) and where the $\omega_{m, A}(x, d x)$ are spacetime forms. Furthermore, if

$$
\sum_{m, A} \omega_{m, A}(x, d x) \alpha_{m}^{k_{m}, 0}\left(e_{\mu}{ }^{a}, s e_{\mu}{ }^{a}\right) P_{A}\left(\mathcal{T}, \mathcal{T}^{*}, \hat{\xi}, \hat{C}\right)=s b
$$

holds for some local form $b$, then all the form-coefficients $\omega_{m, A}(x, d x)$ vanish. [Finiteness of the sums in (5.5) and (5.0) is understood since the forms are local by assumption].

\section{Descent equations}

The relationship between $\alpha_{m}^{0, k_{m}}$ and $\alpha_{m}^{k_{m}, 0}$ found above provides a good example of the descent equations techniques [27]. These play a crucial role in our investigation of $H^{*, *}(s \mid d)$ and are developed in detail in this section. Let $a^{k, q}$ be a local $q$-form of ghost number $k$. Assume that $a^{k, q}$ is a non-trivial solution of the Wess-Zumino consistency condition,

$$
\begin{gathered}
s a^{k, q}+d a^{k+1, q-1}=0, \\
a^{k, q} \neq s b^{k-1, q}+d b^{k, q-1} .
\end{gathered}
$$

By applying $s$ to (6.1), one gets $d\left(s a^{k+1, q-1}\right)=0$. Thus, $s a^{k+1, q-1}$ is a closed local form. It follows from 4.5) that $s a^{k+1, q-1}+d a^{k+2, q-2}=\sum \lambda_{m} \alpha_{m}^{0, k_{m}}$, where the $\lambda_{m}$ can be non-vanishing only if $k+2=0$. But even in that case, theorem 5.2 implies $\lambda_{m}=0$. Thus, $a^{k+1, q-1}$ is itself also a solution of the Wess-Zumino consistency condition,

$$
s a^{k+1, q-1}+d a^{k+2, q-2}=0 .
$$


It should be noted that $a^{k+1, q-1}$ is not completely determined by (6.1), even as a representative of $H^{k+1, q-1}(s \mid d)$. Indeed, $a^{k, q}$ itself is determined only up to the addition of trivial solutions, i.e., up to the addition of a $s$-coboundary modulo $d$. The resulting ambiguity in $a^{k+1, q-1}$ reads

$$
a^{k+1, q-1} \rightarrow a^{k+1, q-1}+s b^{k, q-1}+d b^{k+1, q-2}+\sum \lambda_{m} \alpha_{m}^{0, k_{m}}
$$

since the last term in (6.4) disappears from (6.1). The presence of the $\alpha_{m}^{0, k_{m}}$ 's is permitted for $k=-1$ and plays no incidence on the analysis of the descent equations.

Now, two things can happen :

(i) either $a^{k+1, q-1}$ is trivial in $H^{k+1, q-1}(s \mid d)$ modulo a combination of the $\alpha_{m}^{0, k_{m}}$ 's; in this case, one may eliminate it by adding to $a^{k, q}$ a $d$-exact term; once this is done, $a^{k, q}$ is $s$-closed, $s a^{k, q}=0$; one says that $a^{k, q}$ generates a trivial descent;

(ii) or $a^{k+1, q-1}$ is non-trivial in $H^{k+1, q-1}(s \mid d)$, even up to a combination of the $\alpha_{m}^{0, k_{m}}$ 's; in this second case, there is no way to make $a^{k, q} s$-closed by adding to it trivial solutions; we shall show that the descent then keeps going all the way down to the zero forms, where it hits an element of $H^{*}(s, \mathcal{A})$. One says that $a^{k, q}$ generates a non-trivial descent.

The argument for establishing this fundamental property of non-trivial descents proceeds along lines extremely close to those of [7, 19], where, however, only the case $q=n$ is discussed and the extra solutions arising from the cohomology of the vielbein manifold are not considered. It goes as follows. If one acts with $s$ on (6.3) and uses again (4.5) and theorem 5.2, one finds that $a^{k+2, q-2}$ is also a solution of the Wess-Zumino consistency condition. The procedure can be repeated until one hits a form $a^{k+u, q-u}$ that is $s$-closed,

$$
s a^{k+u, q-u}=0, \quad u \leq q,
$$

which one eventually does (at the latest for $u=q$ since the Wess-Zumino condition reduces to (6.5) for 0 -forms). If $a^{k+u, q-u}$ is trivial in $H^{k+u, q-u}(s \mid d)$ modulo a combination of the $\alpha_{m}^{0, k_{m}}$ 's, then one may assume $a^{k+u, q-u}=0$ by adding to $a^{k+u-1, q-u+1}$ a $d$-closed term. The new $a^{k+u-1, q-u+1}$ is clearly $s$ closed, $s a^{k+u-1, q-u+1}=0$. The redefinition does not modify the previous $a^{i, j}$ 's and shortens the descent by one step. We shall assume that such redefinitions have already been made. Therefore, the last form $a^{k+u, q-u}$ in the descent is non-trivial in $H^{k+u, q-u}(s \mid d)$, even modulo combinations of the $\alpha_{m}^{0, k_{m}}$ 's, and 
thus certainly non-trivial in $H^{*}(s, \mathcal{E})$. By assumption, we have also $u>0$ (non-trivial descent).

According to theorem 5.3, $a^{k+u, q-u}$ takes the form

$$
a^{k+u, q-u}=\sum_{m, A} \omega_{m, A}(x, d x) \alpha_{m}^{k_{m}, 0}\left(e_{\mu}{ }^{a}, s e_{\mu}{ }^{a}\right) P_{A}\left(\mathcal{T}, \mathcal{T}^{*}, \hat{\xi}, \hat{C}\right)
$$

modulo $s$-trivial terms that can be absorbed through redefinitions. The crucial observation at this point is that all the elements $\alpha_{m}^{k_{m}, 0} P_{A}$ of $H^{*}(s, \mathcal{A})$ also come from a non-trivial descent, i.e., their $d$ variation is $s$-exact. This was seen to be true for $\alpha_{m}^{k_{m}, 0}$ already in the previous section but holds in fact for any BRST invariant local function due to a general property of gravitational theories. Namely the presence of diffeomorphisms among the gauge transformations implies that the derivative operator $\partial_{\mu}$ can be represented on the fields, antifields and their derivatives by $\partial_{\mu}=s b_{\mu}+b_{\mu} s$ with $b_{\mu}=\partial / \partial \xi^{\mu}$. Therefore the exterior derivative of a local function can be represented by a commutator:

$$
\alpha \in \mathcal{A} \quad \Rightarrow \quad d \alpha=[b, s] \alpha
$$

where $b$ is the operator

$$
b=d x^{\mu} \frac{\partial}{\partial \xi^{\mu}} .
$$

Of course it is understood in (6.7) that $\alpha$ is expressed in terms of the orginial ghosts $\xi^{\mu}$ and $C^{I}$ rather than in terms of the $\hat{\xi}^{a}$ and $\hat{C}^{I}$. In particular (6.7) implies

$$
d\left(\alpha_{m}^{k_{m}, 0} P_{A}\right)=s \sigma_{m A}, \quad \sigma_{m A}=-b\left(\alpha_{m}^{k_{m}, 0} P_{A}\right)
$$

and thus

$$
d a^{k+u, q-u}=s \sum_{m, A} \omega_{m, A}(x, d x) \sigma_{m A}+\sum_{m, A}\left(d \omega_{m, A}(x, d x)\right) \alpha_{m}^{k_{m}, 0} P_{A} .
$$

But $d a^{k+u, q-u}$ is $s$-exact since, by assumption, $a^{k+u, q-u}$ comes from a nontrivial descent. Accordingly, we get

$$
\sum_{m, A}\left(d \omega_{m, A}(x, d x)\right) \alpha_{m}^{k_{m}, 0} P_{A}=s \text { (something), }
$$

which by theorem 5.3 is possible only if $d \omega_{m, A}$ vanishes. Thus the coefficient forms $\omega_{m, A}$ of $\alpha_{m}^{k_{m}, 0} P_{A}$ in (6.6) are closed forms. Hence, if $q-u>0$, the forms 
$\omega_{m, A}$ are exact (Poincaré lemma on $\mathbf{R}^{n}$ ). But then one gets, using (6.6) and (6.9), that $a^{k+u, q-u}$ is trivial in $H^{k+u, q-u}(s \mid d)$, contrary to the assumption. Thus $q=u$, and the coefficients $\omega_{m, A}$ in the expansion (6.6) are constants,

$$
a^{k+q, 0}=\sum_{m, A} \lambda_{m, A} \alpha_{m}^{k_{m}, 0}\left(e_{\mu}{ }^{a}, s e_{\mu}{ }^{a}\right) P_{A}\left(\mathcal{T}, \mathcal{T}^{*}, \hat{\xi}, \hat{C}\right), \quad \lambda_{m, A}=\text { const } .
$$

This indicates that indeed any non-trivial descent ends on a zero-form in the algebra $\mathcal{A}$, which is a non-trivial $s$-cocycle. Conversely there are no obstructions to lift any $s$-cocycle $\alpha^{g} \in \mathcal{A}$ to solutions $a^{g-q, q}$ of (6.1) given by

$$
a^{g-q, q}=\frac{1}{q !} b^{q} \alpha^{g}
$$

due to (6.7). Thus the "integration" of the descent equations is trivial. Note however that the tower of forms obtained from (6.13) terminates at some form degree $q=q_{\max }$ which may be smaller than the spacetime dimension (viewing $\alpha^{g}$ as a polynomial in the undifferentiated $\xi^{\mu}, q_{\max }$ is the degree of that polynomial). In particular one has $d a^{g-q_{\max }, q_{\max }}=0$. For $q_{\max }<n$ we conclude, using (4.5), that $a^{g-q_{\max }, q_{\max }}$ is equivalent to a linear combination of the $\alpha_{m}^{0, k_{m}}$ with $k_{m}=q_{\max }$, and thus that $\alpha^{g}$ is equivalent to a linear combination of the corresponding $\alpha_{m}^{k_{m}, 0}$, cf. (5.3). Furthermore, arguments used already within the derivation of theorem 5.2 show that the $a^{g-q, q}(q=$ $\left.0, \ldots, q_{\max }\right)$ obtained from (6.13) solve (6.1) non-trivially if $\alpha^{g}$ is a non-trivial $s$-cocycle (and vice versa). This proves

Theorem 6.1 Any solution of (1.1) generating a non-trivial descent corresponds via the descent equations to a non-trivial s-cocycle $\alpha \in \mathcal{A}$ and can be obtained from the latter through (6.13). Conversely, any non-trivial scocycle $\alpha \in \mathcal{A}$ gives rise to a non-trivial solution of the descent equations given by the tower of forms obtained from $\alpha$ through (6.13). This tower of forms ranges always from a 0 -form to an $n$-form unless $\alpha$ is equivalent to a linear combination of the $\alpha_{m}^{k_{m}, 0}$ with $k_{m}<n$ (including the constant).

This result implies immediately that a BRST invariant function $\alpha \in \mathcal{A}_{2}$ with ghost number $<n$ is BRST exact up to a constant unless it depends non-trivially on antifields. Indeed, suppose that $\alpha$ is non-trivial. Then it corresponds by theorem 6.1 to a non-trivial volume form or it is equivalent to a constant. The volume form has negative ghost number due to $g h(\alpha)<n$ 
and therefore must depend on antifields. Since the "ascent procedure" (6.13) does not introduce any antifield dependence, we conclude that $\alpha$ involves also antifields or is $s$-exact up to constant.

Corollary 6.1 Any antifield independent BRST invariant function $\alpha \in \mathcal{A}_{2}$ with ghost number $G<n$ is BRST exact (for $G \neq 0$ ) or equivalent to a constant (for $G=0$ ),

$$
\begin{aligned}
& s \alpha=0, \quad \alpha \in \mathcal{A}_{2}, \quad g h(\alpha)=G<n, \quad \operatorname{antigh}(\alpha)=0 \\
& \Rightarrow \quad \alpha=s \beta+\lambda \delta_{G}^{0}, \quad \lambda=\text { const. }
\end{aligned}
$$

So far we have only treated the case in which $a^{k, q}$ generates a non-trivial descent. The case of a trivial descent is easily discussed since $s a^{k, q}=0$ implies according to theorem 5.3 that $a^{k, q}$ is of the form (5.5). We note that the coefficients $\omega_{m, A}$ occurring there can be assumed to be non-exact forms since a contribution $\omega_{m, A} \alpha_{m}^{k_{m}, 0} P_{A}$ can be removed from $a^{k, q}$ through a redefinition $a^{k, q} \rightarrow a^{k, q}+s b^{k-1, q}+d b^{k, q-1}$ whenever $\omega_{m, A}$ is exact as a consequence of (6.9). In particular, non-trivial volume forms $a^{k, q}$ have always a non-trivial descent since all volume forms $\omega_{m, A}(x, d x)$ are exact (Poincaré lemma in $\mathbf{R}^{n}$ ). We thus obtain

Theorem 6.2 Any non-trivial solution of (1.1) generating a trivial descent has the form (5.5) where all coefficient forms $\omega_{m, A}(x, d x)$ can be assumed to be non-exact. In particular, non-trivial volume forms generate always a non-trivial descent.

Note that, due to the absence of non-trivial volume forms generating a trivial descent, $H^{g, n}(s \mid d)$ is isomorphic to $H^{g+n}(s, \mathcal{A})$ after dividing out the constants,

$$
H^{g-n, n}(s \mid d) \simeq H^{g}(s, \mathcal{A}) / \mathbf{R} .
$$

On the level of the representatives of the respective cohomology classes, this isomorphism is established by (6.13) for $q=n$.

It should be stressed that the crucial property that makes (a) a non-trivial descent go all the way down to a 0 -form $\alpha \in \mathcal{A}$ and (b) the integration of the descent equations trivial, is that $d \alpha$ is $s$-exact whenever $\alpha \in \mathcal{A}$ is 
BRST invariant. This follows from (6.7), which reflects the presence of the diffeomorphisms among the gauge symmetries, and is of course not true for a generic gauge theory as the example of electromagnetism shows. There the BRST transformation of the gauge fields $A_{\mu}$ and the ghost field $C$ are $s A_{\mu}=$ $\partial_{\mu} C$ and $s C=0$. Hence, the field strengths $F_{\mu \nu}$ are $s$-invariant but $d F_{\mu \nu}$ is not $s$-exact (for $n>2$ ). As a consequence, in electromagnetism a non-trivial descent does not necessarily terminate with a 0 -form. A counterexample is the abelian Chern-Simons 3 -form $\alpha^{0,3}=A d A$ where $A=d x^{\mu} A_{\mu}$ : it is indeed non-trivial but the descent equations arising from it terminate with a 2 -form $\alpha^{1,2}=C d A$ due to $s \alpha^{0,3}+d \alpha^{1,2}=0, s \alpha^{1,2}=0$. Furthermore, in contrast to gravitational theories, there are non-trivial volume forms which have a trivial descent, as e.g. $d^{n} x C F_{\mu \nu}$. Of course, this does not exclude the existence of an operator $b$ which satisfies (6.7) on particular solutions of the descent equations [28].

Nevertheless the descent equations that one encounters in electromagnetism or Yang-Mills theory show up in gravitational theories too, within a decomposition of the last equation $s a^{k+q, 0}=0$ of the gravitational descent equations into parts with definite degree in the ghosts $\xi^{\mu}$. In this decomposition the $\xi^{\mu}$ play a role similar to the differentials in the descent equations of non-gravitational theories, such as electromagnetism or Yang-Mills theory (indeed we will use a similar decomposition in section 7 to compute $\left.H^{*}\left(s, \mathcal{A}_{2}\right)\right)$. One may also take the point of view that it is the sum $d x^{\mu}+\xi^{\mu}$ which in gravitational theories takes the part of the differentials in nongravitational theories $[7,19,21,29$.

\section{$7 \quad$ BRST cohomology in $\mathcal{A}_{2}$}

\section{$7.1 \quad$ Notation}

In this subsection we prepare the computation and presentation of $H^{*}\left(s, \mathcal{A}_{2}\right)$ by defining some functions which we will encounter later.

First we introduce polynomials $\theta_{(K)}$ in the ghosts representing the socalled primitive elements of the Lie algebra cohomology of $\mathcal{G}$. They correspond bijectively to the independent Casimir operators of $\mathcal{G}$ and therefore their number equals the rank of $\mathcal{G}$,

$$
(K)=1, \ldots, \operatorname{rank}(\mathcal{G}) .
$$


One can construct the $\theta_{(K)}$ explicitly by means of suitable matrix representations $\left\{T_{I}^{(K)}\right\}$ of $\mathcal{G}$ 30, 31, 32,

$$
\theta_{(K)}(\hat{C})=(-)^{m+1} \frac{m !(m-1) !}{(2 m-1) !} \operatorname{tr}\left(\hat{\mathcal{C}}_{(K)}^{2 m-1}\right), \quad m=m_{(K)}, \quad \hat{\mathcal{C}}_{(K)}=\hat{C}^{I} T_{I}^{(K)}
$$

where $m_{(K)}$ denotes the order of the Casimir operator corresponding to $\theta_{(K)}$. Using (3.16) one can extend (7.1) to a Chern-Simons polynomial in the ghosts $\hat{C}^{I}$ and the curvature-quantities (3.17) 32,

$$
q_{(K)}=\sum_{k=0}^{m-1}(-)^{k} \frac{m !(m-1) !}{(m+k) !(m-k-1) !} \operatorname{Str}\left(\hat{\mathcal{C}} \hat{\mathcal{B}}^{k} \hat{\mathcal{F}}^{m-k-1}\right), \quad \hat{\mathcal{B}}=\hat{\mathcal{C}}^{2}
$$

where Str denotes the symmetrized trace of matrices (cf. appendix $\mathrm{A}$ ) and on the r.h.s. it is understood that

$$
\hat{\mathcal{C}}=\hat{\mathcal{C}}_{(K)}, \quad \hat{\mathcal{F}}=\hat{\mathcal{F}}_{(K)}=\hat{F}^{I} T_{I}^{(K)}, \quad m=m_{(K)} .
$$

(7.2) contains (7.1) and has been constructed such that its BRST transformation does not depend on the $\hat{C}^{I}$ at all,

$$
s q_{(K)}=\operatorname{tr}\left(\hat{\mathcal{F}}_{(K)}^{m_{(K)}}\right)=: f_{(K)} .
$$

Note that $f_{(K)}$ vanishes for $m_{(K)}>n / 2$ since the $\hat{F}^{I}$ are 2- "forms" in the $\hat{\xi}^{a}$. The (non-vanishing) $f_{(K)}$ provide a basis for the $\delta_{I^{-} \text {-invariant polynomials }}$ constructable of the $\hat{F}^{I}$ (the $f_{(K)}$ are not a basis in the vector space sense),

$$
\delta_{I} P(\hat{F})=0 \quad \Leftrightarrow \quad P(\hat{F})=\mathcal{P}(f) .
$$

The $\theta_{(K)}$ and $f_{(K)}$ of the Lorentz group are explicitly given in appendix $\mathrm{B}$. The abelian $\delta_{I} \in \mathcal{G}$ provide the $\theta_{(K)}$ and $f_{(K)}$ with $m_{(K)}=1$, given just by the abelian ghosts $\hat{C}^{I}$ and the abelian 2- "forms" $\hat{F}^{I}$ respectively.

Next we introduce a basis $\left\{X_{\Delta}^{*}: \Delta=1,2, \ldots\right\}$ for the vector space of inequivalent solutions of

$$
\delta X_{\Delta}^{*}\left(\mathcal{T}, \mathcal{T}^{*}\right)=D_{a} J_{\Delta}^{a}(\mathcal{T}), \quad \delta_{I} X_{\Delta}^{*}\left(\mathcal{T}, \mathcal{T}^{*}\right)=0, \quad \operatorname{antigh}\left(X_{\Delta}^{*}\left(\mathcal{T}, \mathcal{T}^{*}\right)\right)=1
$$


where two $X^{*}$ 's are called equivalent if they differ by functions of the form $\delta M\left(\mathcal{T}, \mathcal{T}^{*}\right)+D_{a} N^{a}\left(\mathcal{T}, \mathcal{T}^{*}\right)$ since the latter solve (7.5) trivially. Due to $\left[\delta, \delta_{I}\right]=0$ we can assume with no loss of generality the $J_{\Delta}^{a}(\mathcal{T})$ to be $\delta_{i^{-}}$ invariant local functions transforming under $\mathcal{G}_{L}$ according to the vector representation,

$$
\delta_{i} J_{\Delta}^{a}(\mathcal{T})=0, \quad l_{a b} J_{c \Delta}(\mathcal{T})=\eta_{b c} J_{a \Delta}(\mathcal{T})-\eta_{a c} J_{b \Delta}(\mathcal{T}),
$$

since all pieces of the $J_{\Delta}^{a}$ which do not satisfy (7.6) must result in contributions to $D_{a} J_{\Delta}^{a}$ which cancel separately. We now define functions $G_{\Delta}^{*}$ in terms of the solutions of (7.5) according to

$$
G_{\Delta}^{*}=\left(X_{\Delta}^{*}-(-)^{\varepsilon_{\Delta}} J_{\Delta}^{a} \frac{\partial}{\partial \hat{\xi}^{a}}\right) \hat{\Theta}
$$

where $\varepsilon_{\Delta}$ denotes the grading of $J_{\Delta}^{a}$ f and $\hat{\Theta}$ plays the role of the volume element in $\mathcal{A}_{2}$,

$$
\hat{\Theta}=\hat{\xi}^{0} \ldots \hat{\xi}^{n-1}=\frac{(-)^{n+1}}{n !} \varepsilon_{a_{1} \ldots a_{n}} \hat{\xi}^{a_{1}} \ldots \hat{\xi}^{a_{n}} .
$$

$G_{\Delta}^{*}$ is BRST invariant because of (7.5) and (7.6),

$$
s G_{\Delta}^{*}=0 .
$$

We anticipate that the $J_{\Delta}^{a}$ provide the "covariantizable" non-trivial local Noether currents, cf. section 10. Therefore, if one knows the latter for the particular model under consideration (i.e. for the particular choice of the classical action), one can construct all $G_{\Delta}^{*}$ explicitly (and vice versa).

Finally we introduce a label $\alpha$ for those abelian factors of the gauge group under which all the matter fields $y^{m}$ are uncharged,

$$
\left\{\delta_{\alpha}\right\}=\left\{\delta_{I}:\left[\delta_{I}, \delta_{J}\right]=0 \forall J \wedge \delta_{I} y^{m}=0 \forall m\right\} .
$$

The corresponding gauge fields will be called "free" henceforth although they generally couple to other fields (in particular to the metric). The BRST transformation of the antifields $C_{\alpha}^{*}$ following from (2.7) is given by

$$
s C_{\alpha}^{*}=\partial_{\mu}\left(\xi^{\mu} C_{\alpha}^{*}-A_{\alpha}^{* \mu}\right) .
$$

\footnotetext{
${ }^{4}$ With no loss of generality we assume $X_{\Delta}^{*}$ and $J_{\Delta}^{a}$ to have definite (but opposite) grading.
} 
This shows that the volume forms $d^{n} x C_{\alpha}^{*}$ are solutions $a^{-2, n}$ of (1.1). In fact they are even non-trivial according to [14], section 13. Hence, they correspond via descent equations (cf. section 6) to BRST invariant 0-forms with ghost number $(n-2)$ which we denote by $q_{\alpha}^{*}$,

$$
s q_{\alpha}^{*}=0 .
$$

Contrary to (7.11), the explicit form of the $q_{\alpha}^{*}$ is not universal but depends on the particular choice of the classical action. However, from (7.11) we can conclude that the $q_{\alpha}^{*}$ can be always chosen to be in $\mathcal{A}_{2}$ and of the form

$$
q_{\alpha}^{*}=\hat{C}_{\alpha}^{*} \hat{\Theta}-\hat{A}_{\alpha}^{* a} \frac{\partial \hat{\Theta}}{\partial \hat{\xi}^{a}}+\ldots \in \mathcal{A}_{2}
$$

where the non-written terms have degrees $\leq n-2$ in the $\hat{\xi}$. For instance, (2.11) yields

$$
q_{\alpha}^{*} \stackrel{(2.11)}{=}\left(\hat{C}_{\alpha}^{*}-\hat{A}_{\alpha}^{* a} \frac{\partial}{\partial \hat{\xi}^{a}}+\frac{1}{2} F_{\alpha}^{a b} \frac{\partial}{\partial \hat{\xi}^{a}} \frac{\partial}{\partial \hat{\xi}^{b}}\right) \hat{\Theta} .
$$

\subsection{Computation of $H^{*}\left(s, \mathcal{A}_{2}\right)$}

To compute $H^{*}\left(s, \mathcal{A}_{2}\right)$ we have to solve

$$
s \alpha=0, \quad \alpha \in \mathcal{A}_{2}
$$

modulo trivial solutions $s \beta\left(\beta \in \mathcal{A}_{2}\right)$. Two solutions are called equivalent if they differ by a trivial solution. To solve (7.15) we decompose it into parts of definite degree in the ghosts $\hat{\xi}^{a}$ and call this degree $\hat{\xi}$-degree. The part of $\hat{\xi}$-degree $p$ contained in $\alpha$ is denoted by $\alpha_{p}$,

$$
\alpha=\sum_{p=\ell}^{n} \alpha_{p}, \quad N_{\hat{\xi}} \alpha_{p}=p \alpha_{p}
$$

where $\alpha_{\ell} \neq 0$ is understood and $N_{\hat{\xi}}$ denotes the counting operator for the $\hat{\xi}$,

$$
N_{\hat{\xi}}=\hat{\xi}^{a} \frac{\partial}{\partial \hat{\xi}^{a}} .
$$


The smallest $\hat{\xi}$-degree $\ell$ occurring in this decomposition is called the level of $\alpha$. It provides a simple but useful prescription for the choice of representatives of the cohomology classes of $H^{*}\left(s, \mathcal{A}_{2}\right)$ : out of two equivalent non-trivial solutions of $(7.15)$ with different levels we choose always the one with higher level.

The BRST operator decomposes into parts whose $\hat{\xi}$-degrees range from 0 to 2 ,

$$
s=s_{0}+s_{1}+s_{2}, \quad\left[N_{\hat{\xi}}, s_{p}\right]=p s_{p} .
$$

The part $s_{0}$ is given by

$$
s_{0}=\delta+\gamma_{\mathcal{G}},
$$

where $\delta$ is the Koszul-Tate differential whose action in $\mathcal{A}_{2}$ is given by (3.13), and $\gamma_{\mathcal{G}}$ is the longitudinal exterior derivative along the gauge orbits of $G_{L} \times$ $G_{Y M}$ ("BRST operator of $G_{L} \times G_{Y M}$ "). The latter acts in $\mathcal{A}_{2}$ according to

$$
\gamma_{\mathcal{G}}=-\frac{1}{2} \hat{C}^{J} \hat{C}^{K} f_{K J}^{I} \frac{\partial}{\partial \hat{C}^{I}}+\hat{C}^{I} \delta_{I}
$$

where

$$
\delta_{i} \hat{\xi}^{a}=0, \quad l_{a b} \hat{\xi}_{c}=\eta_{b c} \hat{\xi}_{a}-\eta_{a c} \hat{\xi}_{b}, \quad \delta_{I} \hat{C}^{J}=-f_{I K}{ }^{J} \hat{C}^{K}
$$

i.e. explicitly one has $\gamma_{\mathcal{G}} \hat{C}^{I}=\frac{1}{2} \hat{C}^{J} \hat{C}^{K} f_{K J}^{I}$ and $\gamma_{\mathcal{G}} Y=\hat{C}^{I} \delta_{I} Y$ for $Y \in$ $\left\{\hat{\xi}^{a}, \mathcal{T}^{r}, \mathcal{T}_{\bar{r}}^{*}\right\}$. The part $s_{1}$ acts on the generators of $\mathcal{A}_{2}$ according to

$$
s_{1} \mathcal{T}^{r}=\hat{\xi}^{a} D_{a} \mathcal{T}^{r}, \quad s_{1} \mathcal{T}_{\bar{r}}^{*}=\hat{\xi}^{a} D_{a} \mathcal{T}_{\bar{r}}^{*}, \quad s_{1} \hat{\xi}^{a}=s_{1} \hat{C}^{I}=0,
$$

i.e. $s_{1}$ is an exterior covariant derivative in $\mathcal{A}_{2}$ whose "differentials" are the $\hat{\xi}^{a}$ and which vanishes on the $\hat{C}^{I}$. The part $s_{2}$ is non-vanishing only on the ghosts $\hat{C}^{I}$ and transforms the latter into the curvature-quantities (3.17), i.e. it is in $\mathcal{A}_{2}$ given by the operator

$$
s_{2}=\hat{F}^{I} \frac{\partial}{\partial \hat{C}^{I}}
$$

We note that $\delta, \gamma_{\mathcal{G}}, s_{1}$ and $s_{2}$ have trivial anti-commutation relations apart from

$$
\gamma_{\mathcal{G}} s_{2}+s_{2} \gamma_{\mathcal{G}}+s_{1}^{2}=0
$$


We obtain the following decomposition of $(7.15)$ relative to the $\hat{\xi}$-degree:

$$
\begin{aligned}
& 0=s_{0} \alpha_{\ell} \\
& 0=s_{0} \alpha_{\ell+1}+s_{1} \alpha_{\ell}, \\
& 0=s_{0} \alpha_{p+2}+s_{1} \alpha_{p+1}+s_{2} \alpha_{p} \text { for } \ell \leq p \leq n-2 .
\end{aligned}
$$

Here we used that the $\hat{\xi}$-degree cannot exceed $n$ since the $\hat{\xi}$ 's anticommute.

We shall now analyse (7.15) starting from (7.24). To that end we need the cohomology of $s_{0}$ in $\mathcal{A}_{2}$ :

Lemma 7.1 The cohomology classes of $H^{*}\left(s_{0}, \mathcal{A}_{2}\right)$ are represented by linearly independent polynomials in the ghost-polynomials (7.1) whose coefficient functions are $\delta_{I}$-invariant polynomials in the $\hat{\xi}^{a}$ and $\mathcal{T}^{r}$,

$$
\begin{aligned}
& s_{0} \alpha=0, \quad \alpha \in \mathcal{A}_{2} \Leftrightarrow \alpha=\alpha^{i}(\hat{\xi}, \mathcal{T}) \omega_{i}(\hat{C})+s_{0} \beta, \\
& \delta_{I} \alpha^{i}(\hat{\xi}, \mathcal{T})=0, \quad \omega_{i}(\hat{C})=P_{i}(\theta(\hat{C})), \quad \beta \in \mathcal{A}_{2} .
\end{aligned}
$$

Proof. We decompose $\alpha$ into parts $\alpha^{k}$ with definite degree $k$ in the $\hat{C}^{I}$ (" $\hat{C}$-degree"), $\alpha=\sum_{k=0}^{\kappa} \alpha^{k}$. Note that $\kappa$, the highest occurring $\hat{C}$-degree, cannot exceed $\operatorname{dim}(\mathcal{G})$ as the $\hat{C}^{I}$ anticommute. Since the parts $\delta$ and $\gamma_{\mathcal{G}}$ of $s_{0}$ have $\hat{C}$-degree 0 and 1 respectively, the condition $s_{0} \alpha=0$ implies in particular $\gamma_{\mathcal{G}} \alpha^{\kappa}=0$. Using the Lie algebra cohomology of $\mathcal{G}$ (cf. appendix B for details), we conclude $\alpha^{\kappa}=\bar{\alpha}^{i_{\kappa}} \omega_{i_{\kappa}}+\gamma_{\mathcal{G}} \bar{\beta}^{\kappa-1}, \delta_{I} \bar{\alpha}^{i_{\kappa}}=0$ where $\bar{\alpha}^{i_{\kappa}}=$ $\bar{\alpha}^{i_{\kappa}}\left(\hat{\xi}, \mathcal{T}, \mathcal{T}^{*}\right)$, and the $\omega_{i_{\kappa}}=P_{i_{\kappa}}(\theta(\hat{C}))$ are linearly independent and have $\hat{C}$ degree $\kappa$. We now insert this result for $\alpha^{\kappa}$ in $\gamma_{\mathcal{G}} \alpha^{\kappa}+\delta \alpha^{\kappa+1}=0$, which is also implied by $s_{0} \alpha=0$, and obtain $\left(\delta \bar{\alpha}^{i_{\kappa}}\right) \omega_{i_{\kappa}}+\gamma_{\mathcal{G}}\left(\alpha^{\kappa-1}-\delta \bar{\beta}^{\kappa-1}\right)=0$. Using the Lie algebra cohomology of $\mathcal{G}$ again, we conclude $\delta \bar{\alpha}^{i_{\kappa}}=0$. The acyclicity of $\delta$ in positive antighost number (cf. [20] and appendices $\mathrm{O}$ and D for details) implies $\bar{\alpha}^{i_{\kappa}}=\alpha^{i_{\kappa}}+\delta \beta^{i_{\kappa}}$ where $\alpha^{i_{\kappa}}=\alpha^{i_{\kappa}}(\hat{\xi}, \mathcal{T})$ and $\beta^{i_{\kappa}}=\beta^{i_{\kappa}}\left(\hat{\xi}, \mathcal{T}, \mathcal{T}^{*}\right)$ are $\delta_{I^{-}}$ invariant polynomials. Now we consider $\hat{\alpha}:=\alpha-\alpha^{i_{\kappa}} \omega_{i_{\kappa}}-s_{0}\left(\bar{\beta}^{\kappa-1}+\beta^{i_{\kappa}} \omega_{i_{\kappa}}\right)$. $\hat{\alpha}$ is $s_{0}$-invariant due to $s_{0} \alpha=s_{0}\left(\alpha^{i_{\kappa}} \omega_{i_{\kappa}}\right)=s_{0}^{2}=0$ and decomposes into parts with $\hat{C}$-degrees $<\kappa . \hat{\alpha}$ is now analysed as $\alpha$ and the arguments are repeated until the highest occurring $\hat{C}$-degree has dropped to zero and one has proved the lemma.

Now, we can always remove any piece of the form $s_{0} \beta_{\ell}$ from $\alpha_{\ell}$ by subtracting the trivial solution $s \beta_{\ell}$ from $\alpha$ without lowering the level of $\alpha$. Therefore 
we conclude from (7.24) by means of lemma 7.1 that we can assume with no loss of generality and in accordance with our prescription for selecting representatives in $H^{*}\left(s, \mathcal{A}_{2}\right)$ that

$$
\alpha_{\ell}=\alpha_{\ell}^{i}(\hat{\xi}, \mathcal{T}) \omega_{i}(\hat{C}), \quad \delta_{I} \alpha_{\ell}^{i}(\hat{\xi}, \mathcal{T})=0, \quad \omega_{i}(\hat{C})=P_{i}(\theta(\hat{C})) .
$$

Inserting (7.28) in (7.25), we obtain $\left(s_{1} \alpha_{\ell}^{i}\right) \omega_{i}+\left(\delta+\gamma_{\mathcal{G}}\right) \alpha_{\ell+1}=0$. Now, $\left(s_{1} \alpha_{\ell}^{i}\right) \omega_{i}$ does not contain any non-vanishing $\gamma_{\mathcal{G}}$-exact piece according to the Lie algebra cohomology of $\mathcal{G}$, cf. (B.2) (note that $\delta_{I} \alpha_{\ell}^{i}=0$ implies $\delta_{I}\left(s_{1} \alpha_{\ell}^{i}\right)=$ $0)$. Therefore we conclude that $\alpha_{\ell+1}$ contains a piece $\alpha_{\ell+1}^{i} \omega_{i}$ such that

$$
s_{1} \alpha_{\ell}^{i}+\delta \alpha_{\ell+1}^{i}=0 \quad \Leftrightarrow \quad s_{1} \alpha_{\ell}^{i} \approx 0 .
$$

Furthermore we can assume

$$
\alpha_{\ell}^{i} \neq s_{1} \beta_{\ell-1}^{i}+\delta \beta_{\ell}^{i} \Leftrightarrow \alpha_{\ell}^{i} \not \approx s_{1} \beta_{\ell-1}^{i}
$$

with no loss of generality since otherwise we can remove the piece containing $\alpha_{\ell}^{i}$ from $\alpha_{\ell}$ by subtracting from $\alpha$ the trivial piece $s\left[\left(\beta_{\ell-1}^{i}+\beta_{\ell}^{i}\right) \omega_{i}\right]$ (no sum over $i$ here). Note that this does not lower the level of $\alpha$ since $\beta_{\ell-1}^{i}$ and $\beta_{\ell}^{i}$ have antighost number 0 and 1 respectively and are $\delta_{I}$-invariant with no loss of generality due to antigh $\left(\alpha_{\ell}^{i}\right)=\delta_{I} \alpha_{\ell}^{i}=0$ and $\left[\delta_{I}, s_{1}\right]=\left[\delta_{I}, \delta\right]=0$.

The solution of (7.29), (7.30) is one of the crucial steps within the computation. It is interesting in its own right since it provides the weak equivariant cohomology of $d$ (cf. section 8 for details). In fact it is an on-shell version of the "gravitational covariant Poincaré lemma" of [7]. The latter provides the (strong) equivariant cohomology of $d$ for Einstein-Yang-Mills theories stating that the corresponding cohomology classes are in form degrees $<n$ represented by invariant polynomials in the curvature 2 -forms $R^{a b}=\frac{1}{2} d x^{\mu} d x^{\nu} R_{\mu \nu}^{a b}$ and $F^{i}=\frac{1}{2} d x^{\mu} d x^{\nu} F_{\mu \nu}{ }^{i}$. D Recalling the results of 14 for the characteristic cohomology of $d$ (in the space of all local forms), it is not too surprising that in all form degrees $<n-2$ the weak and strong equivariant cohomology of $d$ agree whereas they differ in form degrees $\geq n-2$ :

Theorem 7.1 (Weak covariant Poincaré lemma)

(i) $\delta_{I}$-invariant functions $\alpha(\hat{\xi}, \mathcal{T})$ which are weakly $s_{1}$-closed are weakly $s_{1}$-exact up to invariant polynomials in the $\hat{F}^{I}$, functions of maximal $\hat{\xi}$-degree

\footnotetext{
${ }^{5}$ Analogous results have been derived already in 33] for pure gravity (Riemannian manifolds) and in 25] for Yang-Mills theory.
} 
and the antifield independent parts of the $G_{\Delta}^{*}$ and of those $q_{\alpha}^{*}$ which do not depend on the $\hat{C}^{I}$,

$$
\begin{aligned}
& s_{1} \alpha(\hat{\xi}, \mathcal{T}) \approx 0, \quad \delta_{I} \alpha(\hat{\xi}, \mathcal{T})=0 \\
\Leftrightarrow \quad & \alpha \approx P(\hat{F})+\left.\lambda^{\alpha^{0}} q_{\alpha^{0}}^{*}\right|_{\Phi^{*}=0}+\left.\lambda^{\Delta} G_{\Delta}^{*}\right|_{\Phi^{*}=0}+\hat{\Theta} L(\mathcal{T})+s_{1} \beta
\end{aligned}
$$

where $\beta=\beta(\hat{\xi}, \mathcal{T})$ and

$$
\begin{aligned}
& \delta_{I} P(\hat{F})=\delta_{I} L(\mathcal{T})=\delta_{I} \beta(\hat{\xi}, \mathcal{T})=0 \\
& \left\{q_{\alpha^{0}}^{*}\right\}=\left\{q_{\alpha}^{*}: \partial q_{\alpha}^{*} / \partial \hat{C}^{I}=0\right\} .
\end{aligned}
$$

(ii) No non-vanishing $\delta_{I}$-invariant polynomial $P(\hat{F})$ with ghost number $<n-1$ is weakly $s_{1}$-exact,

$$
d_{I_{1} \ldots I_{k}} \hat{F}^{I_{1}} \ldots \hat{F}^{I_{k}} \approx s_{1} \beta, \quad 2 k<n-1 \quad \Leftrightarrow \quad d_{I_{1} \ldots I_{k}}=0
$$

where the $d_{I_{1} \ldots I_{k}}$ denote totally symmetric constant tensors of $\mathcal{G}$. (7.32) remains valid for $2 k=n-1$ if all $q_{\alpha}^{*}$ can be assumed to independent of the $\hat{C}^{I}$, i.e. if $\left\{q_{\alpha}^{*}\right\}=\left\{q_{\alpha^{0}}^{*}\right\}$.

The proof of this important theorem is given in appendix $\mathbb{E}$. Here we just note that, apart from a refined version of the above-mentioned results derived in [14], the part $s_{2}$ of the BRST operator plays an important role within the proof. By restricting the proof to antifield independent functions, it provides the strong $s_{1}$-cohomology, i.e. an alternative (improved) proof of the (strong) covariant Poincaré lemma which does not use the split $e_{\mu}{ }^{a}=\delta_{\mu}^{a}+h_{\mu}^{a}$, contrary to the original proof given in [7].

We shall now complete the analysis assuming that there are no "free" abelian gauge fields and that (7.32) holds even for $2 k=n$,

$$
2 k=n: \quad d_{I_{1} \ldots I_{k}} \hat{F}^{I_{1}} \ldots \hat{F}^{I_{k}} \approx s_{1} \beta \quad \Leftrightarrow \quad d_{I_{1} \ldots I_{k}}=0 .
$$

That is, we assume that the characteristic classes of degree $n$ remain nontrivial in the equivariant cohomology of $d$ even on-shell. In section 9 we comment this assumption and illustrate that it is physically reasonable. We also show there that it is equivalent to the absence of Chern-Simons forms in (non-trivial) Noether currents. We can now prove: 
Theorem 7.2 If there are no "free" abelian gauge fields and if (7.39) is valid, then one has

$$
s \alpha=0, \alpha \in \mathcal{A}_{2} \quad \Leftrightarrow \quad \alpha=s \beta+\hat{\Theta} L_{\tau}(\mathcal{T}) \omega^{\tau}(\hat{C})+P(q, f)+G_{\Delta}^{*} \omega^{\Delta}(\hat{C})
$$

where the $L_{\tau}(\mathcal{T})$ are $\delta_{I}$-invariant polynomials in the $\mathcal{T}^{r}, \omega^{\tau}(\hat{C})$ and $\omega^{\Delta}(\hat{C})$ are polynomials in the ghost polynomials (7.1), and $P(q, f)$ are BRST invariant polynomials in the "Chern-Simons polynomials" (7.9) and the "Chern polynomials" (7.9),

$$
\delta_{I} L_{\tau}(\mathcal{T})=0, \quad \omega^{\tau}(\hat{C})=P^{\tau}(\theta(\hat{C})), \quad \omega^{\Delta}(\hat{C})=P^{\Delta}(\theta(\hat{C})), \quad s P(q, f)=0 .
$$

Furthermore, under the same assumptions as above (absence of "free" abelian gauge fields and validity of (7.33)) a polynomial $P(q, f)$ is s-exact if and only if it is s-exact in the space of polynomials in the $q$ 's and $f$ 's,

$$
P(q, f)=s \beta \quad \Leftrightarrow \quad P(q, f)=s Q(q, f) .
$$

Proof. Assume that $\alpha$ represents a non-trivial cohomology class of $H^{*}\left(s, \mathcal{A}_{2}\right)$ and has level $\ell$. Then its part $\alpha_{\ell}$ has the form (7.28) where the $\alpha_{\ell}^{i}$ are determined by theorem 7.1 .

Since by assumption there are no $q_{\alpha}^{*}$, part (i) of theorem 7.1 implies in all cases $\ell \leq n-2$ that we can assume the $\alpha_{\ell}^{i}$ to be invariant polynomials in the $\hat{F}^{I}$ (which implies that $\ell$ is even),

$$
\ell \leq n-2: \quad \alpha_{\ell}=P_{\ell}^{i}(\hat{F}) \omega_{i}(\hat{C}), \quad \delta_{I} P_{\ell}^{i}(\hat{F})=0, \quad \omega_{i}(\hat{C})=P_{i}(\theta(\hat{C})) .
$$

We complete now all $\theta_{(K)}(\hat{C})$ occurring in (7.37) to the corresponding $q_{(K)}$ introduced in (7.2) and consider

$$
\beta:=\alpha-P_{\ell}^{i}(\hat{F}) P_{i}(q) \text {. }
$$

Note that, by construction, $\beta$ has higher level than $\ell$,

$$
\beta=\sum_{p \geq \ell(\beta)} \beta_{p}, \quad N_{\hat{\xi}} \beta_{p}=p \beta_{p}, \quad \ell(\beta)>\ell .
$$

Since $\alpha$ is BRST invariant (by assumption), the BRST transformation of $\beta$ is given by

$$
s \beta=-s\left[P_{\ell}^{i}(\hat{F}) P_{i}(q)\right]=:-\mathcal{P}(q, f)
$$


where we used (7.3) and (7.4). Since $s$ contains no part with negative $\hat{\xi}$ degree, $\mathcal{P}(q, f)$ has level $p_{0} \geq \ell(\beta)$,

$$
\mathcal{P}(q, f)=\sum_{p \geq p_{0}} \mathcal{P}_{p}, \quad N_{\hat{\xi}} \mathcal{P}_{p}=p \mathcal{P}_{p}, \quad p_{0} \geq \ell(\beta)
$$

(7.40) implies

$$
\begin{aligned}
-\mathcal{P}_{p_{0}} & =s_{0} \beta_{p_{0}}+s_{1} \beta_{p_{0}-1}+s_{2} \beta_{p_{0}-2}, \\
0 & =s_{0} \beta_{p_{0}-1}+s_{1} \beta_{p_{0}-2}+s_{2} \beta_{p_{0}-3}, \\
& \vdots \\
0 & =s_{0} \beta_{\ell(\beta)+1}+s_{1} \beta_{\ell(\beta)}, \\
0 & =s_{0} \beta_{\ell(\beta)} .
\end{aligned}
$$

We distinguish the cases $\ell(\beta) \geq p_{0}-1$ and $\ell(\beta)<p_{0}-1$. Assume first that $\ell(\beta) \geq p_{0}-1$. Then $(7.42)-(7.45)$ reduce to

$$
-\mathcal{P}_{p_{0}}=s_{0} \beta_{p_{0}}+s_{1} \beta_{p_{0}-1}, \quad 0=s_{0} \beta_{p_{0}-1}
$$

Now, $\mathcal{P}_{p_{0}}$ is of the form

$$
\mathcal{P}_{p_{0}}=P_{p_{0}}^{i}(\hat{F}) \omega_{i}(\hat{C}), \quad \omega_{i}(\hat{C})=P_{i}(\theta(\hat{C}))
$$

where the $P_{p_{0}}^{i}(\hat{F})$ are $\delta_{I^{-}}$invariant polynomials in the $\hat{F}^{I}$ with ghost number $p_{0}$. Using lemma 7.1 and the Lie algebra cohomology of $\mathcal{G}$, we conclude from (7.46) that $\beta_{p_{0}-1}$ and $\beta_{p_{0}}$ must contain parts $\beta_{p_{0}-1}^{i}(\hat{\xi}, \mathcal{T}) \omega_{i}(\hat{C})$ and $\beta_{p_{0}}^{i}\left(\hat{\xi}, \mathcal{T}, \mathcal{T}^{*}\right) \omega_{i}(\hat{C})$ such that

$$
P_{p_{0}}^{i}(\hat{F})=s_{1} \beta_{p_{0}-1}^{i}(\hat{\xi}, \mathcal{T})+\delta \beta_{p_{0}}^{i}\left(\hat{\xi}, \mathcal{T}, \mathcal{T}^{*}\right)
$$

Since this is equivalent to $P_{p_{0}}^{i}(\hat{F}) \approx s_{1} \beta_{p_{0}-1}^{i}$, part (ii) of theorem 7.1 and assumption $(\overline{7.33})$ imply $P_{p_{0}}^{i}(\hat{F})=0$ in $(7.47)$ (note: this is the place where we use $(7.33))$. We conclude $\mathcal{P}(q, f)=0$ and thus that $\beta$ is BRST invariant, cf. (7.40). (7.38) implies now that $\alpha$ agrees with $P_{\ell}^{i}(\hat{F}) P_{i}(q)$ up to a separately BRST invariant function $\beta$ which has higher level than $\alpha$.

In the case $\ell(\beta)<p_{0}-1$ we conclude from (7.44) and (7.45), repeating the arguments that have lead to (7.28), (7.29) and (7.30) and using part (i) 
of theorem 7.1, that we can assume with no loss of generality (by subtracting $s$-exact pieces from $\beta$ if necessary)

$$
\ell(\beta)<p_{0}-1: \quad \beta_{\ell(\beta)}=P_{\ell(\beta)}^{i}(\hat{F}) \omega_{i}(\hat{C})=: \mathcal{Q}(\theta(\hat{C}), f)
$$

since $\ell(\beta)<p_{0}-1$ implies in particular $\ell(\beta)<n-1$. We now complete the $\theta_{(K)}(\hat{C})$ occurring in $\mathcal{Q}$ to the corresponding $q_{(K)}$ and consider the function $\beta^{\prime}:=\beta-\mathcal{Q}(q, f)$ whose level $\ell\left(\beta^{\prime}\right)$ exceeds $\ell(\beta)$ by construction. Since $s \mathcal{Q}(q, f)=\mathcal{P}^{\prime}(q, f)$ is again a polynomial in the $q_{(K)}$ and $f_{(K)}$ due to $(7.3)$, we get

$$
s \beta=\mathcal{P}^{\prime}(q, f)+s \beta^{\prime}, \quad \ell\left(\beta^{\prime}\right)>\ell(\beta) .
$$

(7.40) can now be rewritten in the form

$$
s \beta^{\prime}=-\mathcal{P}(q, f)-\mathcal{P}^{\prime}(q, f) .
$$

We can analyse (7.49) as (7.40) before and repeat the arguments until we arrive at

$$
s \hat{\beta}=-\hat{\mathcal{P}}(q, f), \quad \hat{\beta}=\beta-\mathcal{Q}(q, f)-\mathcal{Q}^{\prime}(q, f)-\ldots, \quad \ell(\hat{\beta}) \geq \hat{p}_{0}-1
$$

where $\ell(\hat{\beta})$ and $\hat{p}_{0}$ are the levels of $\hat{\beta}$ and $\hat{\mathcal{P}}(q, f)=\mathcal{P}(q, f)+\mathcal{P}^{\prime}(q, f)+\ldots$ respectively. This traces the case $\ell(\beta)<p_{0}-1$ made in the distinction after (7.45) back to the case $\ell(\beta) \geq p_{0}-1$ and we conclude $\alpha=P_{\ell}^{i}(\hat{F}) P_{i}(q)+$ $\mathcal{Q}(q, f)+\mathcal{Q}^{\prime}(q, f)+\ldots$ up to a separately BRST invariant function $\hat{\beta}$ whose level exceeds $\ell$.

We conclude that we can assume $\alpha=P(q, f)$ in the cases $\ell \leq n-2$, up to separately BRST invariant functions whose level exceed $\ell$. The latter represent different cohomology classes in accordance with our rule for selecting representatives of the cohomology classes of $H^{*}\left(s, \mathcal{A}_{2}\right)$ (cf. beginning of this subsection). The arguments used within the discussion of (7.40) show also that $\mathcal{P}(q, f)=s \beta$ implies $\beta=\hat{\beta}+\mathcal{Q}(q, f)+\mathcal{Q}^{\prime}(q, f)+\ldots$ where $\hat{\beta}$ is separately $s$-invariant. This proves $(7.36)$.

Consider finally the cases $\ell=n-1$ and $\ell=n$. Part (i) of theorem 7.1 implies that we can assume

$$
\begin{aligned}
\ell=n-1: & \alpha_{n-1}=P_{n-1}^{i}(\hat{F}) \omega_{i}(\hat{C})+\left.G_{\Delta}^{*}\right|_{\Phi^{*}=0} \omega^{\Delta}(\hat{C}) \\
\ell=n: & \alpha_{n}=\hat{\Theta} L_{\tau}(\mathcal{T}) \omega^{\tau}(\hat{C})
\end{aligned}
$$


where the $\omega(\hat{C})$ are polynomials in the ghost polynomials $(7.1)$ and $P_{n-1}^{i}(\hat{F})$ and $L_{\tau}(\mathcal{T})$ are $\delta_{I}$-invariant polynomials (invariant polynomials $P_{n}(\hat{F})$ count among the $\left.L_{\tau}(\mathcal{T})\right)$. Now, $s \theta_{(K)}(\hat{C})=s_{2} \theta_{(K)}(\hat{C})$ has $\hat{\xi}$-degree 2 . Since $P_{n-1}^{i}(\hat{F}), G_{\Delta}^{*}$ and $\hat{\Theta} L_{\tau}(\mathcal{T})$ are BRST invariant and have levels $\geq n-1$, we conclude that (7.52) is already BRST invariant and that $(7.51)$ can be completed to the BRST invariant function $\hat{\alpha}=P_{n-1}^{i}(\hat{F}) \omega_{i}(\hat{C})+G_{\Delta}^{*} \omega^{\Delta}(\hat{C})$. This proves the theorem since it implies in the case $\ell=n-1$ that $\alpha$ agrees with $\hat{\alpha}$ up to a BRST invariant function with level $n$ which is of the form (7.52) modulo an $s$-exact piece.

Thanks to theorem 7.2 one can now complete the analysis by a computation of the BRST cohomology in the space of the polynomials $P(q, f)$ only. This has been done in 31, 32] and we refer to these references for the result. We note that functions $\hat{\Theta} L(\mathcal{T}) P(\theta(\hat{C}))$ are trivial if $L(\mathcal{T}) \approx D_{a} M^{a}(\mathcal{T})$ holds for some $M^{a}(\mathcal{T})$.

Let us finally indicate the modification of the result for $H^{*}\left(s, \mathcal{A}_{2}\right)$ in presence of "free" abelian gauge fields. The latter give rise to additional solutions of (7.15) with level $\ell=n-2$ due to theorem 7.1 and make it necessary to consider this case separately (all other values of $\ell$ can be treated as within the proof of theorem 17.2). The representatives of the resulting additional cohomology classes contain the $q_{\alpha}^{*}$ and have to be evaluated for each classical action separately since the latter determines the explicit form of the $q_{\alpha}^{*}$. We just note that for the action (2.11) the additional cohomology classes are represented by functions

$$
\alpha_{\text {add }} \stackrel{(2.11)}{=} \sum_{\alpha} q_{\alpha}^{*} \frac{\partial P(q)}{\partial \hat{C}^{\alpha}}
$$

where the polynomials $P(q)$ depend only on the $q_{(K)}$ of the semi-simple part of $\mathcal{G}$ and on the "free" abelian ghosts $\hat{C}^{\alpha}$, but not on other abelian ghosts.

\section{Equivariant characteristic cohomology}

We will now show that theorem 7.1 provides indeed the equivariant characteristic cohomology. We define the latter as the weak cohomology of $d$ in the space of local $p$-forms which (a) truly transform as $p$-forms under spacetime diffeomorphisms, (b) are invariant under local Lorentz and Yang-Mills 
gauge transformations, and (c) do not depend on the ghosts, the antifields and (explicitly) on the $x^{\mu}$. Denoting such $p$-forms by

$$
a_{i n v}^{p}=\frac{1}{p !} d x^{\mu_{1}} \ldots d x^{\mu_{p}} \alpha_{\mu_{1} \ldots \mu_{p}},
$$

the requirements (a)-(c) impose that the BRST transformation of the local coefficient functions $\alpha_{\mu_{1} \ldots \mu_{p}}$ coincides with their standard Lie derivative along the diffeomorphism ghost,

$$
\begin{aligned}
s \alpha_{\mu_{1} \ldots \mu_{p}} & =\mathcal{L}_{\xi} \alpha_{\mu_{1} \ldots \mu_{p}} \\
& =\xi^{\nu} \partial_{\nu} \alpha_{\mu_{1} \ldots \mu_{p}}+\sum_{i=1}^{p}\left(\partial_{\mu_{i}} \xi^{\nu}\right) \alpha_{\mu_{1} \ldots \mu_{i-1} \nu \mu_{i+1} \ldots \mu_{p}}
\end{aligned}
$$

The absence of derivatives of the ghosts in (8.2) apart from the first order derivatives of the $\xi^{\mu}$ implies already that $\alpha_{\mu_{1} \ldots \mu_{p}}$ can be written entirely in terms of the tensor fields (3.1) and the undifferentiated $e_{\mu}{ }^{a}$. This is most easily proved using the basis of generators of $\mathcal{A}$ introduced in section 3 . Indeed, (8.2) implies that no generator (3.4) occurs in $s a_{i n v}^{p}$ except for those given by $s e_{\mu}{ }^{a}$ and thus that $a_{i n v}^{p}$ itself does not depend on the generators (3.3) apart from the undifferentiated $e_{\mu}{ }^{a}$. The absence of the undifferentiated ghosts $C^{I}$ in (8.2) then imposes in addition that $\alpha_{\mu_{1} \ldots \mu_{p}}$ is $\delta_{I^{-}}$-invariant and we conclude

$$
\alpha_{\mu_{1} \ldots \mu_{p}}=e_{\mu_{1}}^{a_{1}} \ldots e_{\mu_{p}}^{a_{p}} \alpha_{a_{1} \ldots a_{p}}(\mathcal{T}), \quad \delta_{I} \alpha_{\mu_{1} \ldots \mu_{p}}=0 .
$$

Hence, (8.2) implies (8.3). Conversely, (8.3) implies (8.2) and is therefore equivalent to the latter. Note that (8.3) allows us to express $a_{i n v}^{p}$ in the form

$$
a_{\text {inv }}^{p}=\frac{1}{p !} e^{a_{1}} \ldots e^{a_{p}} \alpha_{a_{1} \ldots a_{p}}(\mathcal{T})
$$

where the $e^{a}$ are the vielbein 1-forms

$$
e^{a}=d x^{\mu} e_{\mu}^{a} .
$$

Due to (2.1) these 1-forms satisfy

$$
D e^{a}=0
$$

where $D$ denotes the exterior covariant derivative

$$
D=e^{b} D_{b}=d x^{\mu}\left(\partial_{\mu}-A_{\mu}{ }^{I} \delta_{I}\right) .
$$


Note that we have defined $D$ without a piece containing the Christoffel connection $\Gamma_{\mu \nu}{ }^{\rho}$. It is not necessary to include such a piece here since it drops out when $D$ acts on any $a_{i n v}^{p}$. In fact even the piece $d x^{\mu} A_{\mu}{ }^{I} \delta_{I}$ drops out due to (8.3). Thus $D$ actually agrees with $d$ on the forms $a_{i n v}^{p}$,

$$
D a_{i n v}^{p}=d a_{i n v}^{p} .
$$

It is now evident that the equivariant characteristic cohomology is indeed described by theorem 7.1 since $D$ turns into $s_{1}$ on functions of the $\mathcal{T}^{r}$ and $e^{a}$ when one substitutes $e^{a} \rightarrow \hat{\xi}^{a}$ and vice versa (recall that $s_{1} \hat{\xi}^{a}=0$, which is analogous to (8.6)). Note that this shows again that the diffeomorphism ghosts play a role similar to the differentials, cf. remarks at the end of section 6. We conclude from theorem 7.1 that the cohomology classes of the equivariant characteristic cohomology are in form degrees $<n-2$ represented by characteristic classes †, i.e. by independent invariant polynomials in the curvature 2 -forms

$$
F^{i}=\frac{1}{2} d x^{\mu} d x^{\nu} F_{\mu \nu}{ }^{i}, \quad F^{a b}=\frac{1}{2} d x^{\mu} d x^{\nu} R_{\mu \nu}^{a b} .
$$

This implies in particular that the equivariant characteristic cohomology is zero in all odd form degrees $<n-2$,

$$
\begin{aligned}
& 2 k<n-3: \quad d a_{i n v}^{2 k+1} \approx 0 \quad \Leftrightarrow \quad a_{i n v}^{2 k+1} \approx d b_{i n v}^{2 k}, \\
& 2 k<n-2: \quad d a_{i n v}^{2 k} \approx 0 \quad \Leftrightarrow \quad a_{i n v}^{2 k} \approx d_{I_{1} \ldots I_{k}} F^{I_{1}} \ldots F^{I_{k}}+d b_{i n v}^{2 k-1} .
\end{aligned}
$$

Moreover, part (ii) of theorem 7.1 implies that no characteristic class with form degree $<n-1$ is trivial in the equivariant characteristic cohomology,

$$
d_{I_{1} \ldots I_{k}} F^{I_{1}} \ldots F^{I_{k}} \approx d b_{i n v}^{2 k-1}, \quad 2 k<n-1 \quad \Rightarrow \quad d_{I_{1} \ldots I_{k}}=0 .
$$

We shall comment this result at the end of this section.

We note that (8.9) and (8.10) remain valid for strong equalities, cf. [7, 33] and the remarks preceding and following theorem 7.1. That is, the strong and the weak equivariant cohomology of $d$ agree in all form degrees $<n-2$. This is not true in form degrees $(n-2)$ and $(n-1)$ since in these cases the equivariant characteristic cohomology contains in general cohomology classes

\footnotetext{
${ }^{6} \mathrm{By}$ an abuse of terminology we call the invariant polynomials in the curvature 2-forms characteristic classes.
} 
which cannot be represented by invariant polynomials in the curvature 2forms (the strong equivariant cohomology of $d$ is in form degrees $(n-2)$ and $(n-1)$ still represented only by characteristic classes). In degree $(n-2)$ there is a difference only if "free" abelian gauge fields are present. They give rise to additional cohomology classes corresponding to the $q_{\alpha^{0}}^{*}$ occurring in (7.31). The explicit form of these $(n-2)$-forms depends on the particular classical action. For the standard Lagrangian (2.11) one has $\left\{q_{\alpha^{0}}^{*}\right\}=\left\{q_{\alpha}^{*}\right\}$ and obtains simply

$$
a_{\alpha}^{n-2} \stackrel{(2.11)}{=} \frac{1}{2(n-2) !}(-)^{n+1} d x^{\mu_{1}} \ldots d x^{\mu_{n-2}} \varepsilon_{\mu_{1} \ldots \mu_{n}} F_{\alpha}^{\mu_{n-1} \mu_{n}} .
$$

The equivariant characteristic cohomology in form degree $(n-1)$ is represented by characteristic classes of this degree (if any) and by forms corresponding to the $G_{\Delta}^{*}$ according to (7.31). The latter are given by

$$
a_{i n v, \Delta}^{n-1}=\frac{1}{(n-1) !} d x^{\mu_{1}} \ldots d x^{\mu_{n-1}} \varepsilon_{\mu_{1} \ldots \mu_{n}} E_{a}^{\mu_{n}} J_{\Delta}^{a}(\mathcal{T}) .
$$

These $(n-1)$-forms are the covariantized Noether currents, cf. section 10 .

Let us finally comment the above results. As we have seen, the strong and weak equivariant cohomology of $d$ can be different only in form degrees $\geq n-2$. This has a deep reason given by the irreducibility of the gauge symmetry. In fact it traces back to an analogous result holding for the characteristic cohomology itself. Indeed we have shown in [14] that the characteristic cohomology vanishes in all non-vanishing form degrees $<n-2-r$ for any normal gauge theory of reducibility order $r$ if the field-antifield manifold is contractible. In our case this remains true except for the occurrence of the topological conserved forms $\alpha_{m}^{0, k_{m}}$, cf. section [10. These forms reflect the non-trivial De Rham cohomology of the vielbein manifold, cf. section 4 , but do not show up in the equivariant characteristic cohomology since they do not satisfy (8.2).

In particular, the irreducibility of the gauge symmetry underlies the result that the characteristic classes of degree $<n-1$ remain non-trivial in the equivariant cohomology of $d$ even on-shell. This is not true, in general, for reducible gauge theories. Indeed, a counterexample is provided by the " $B F$ theory" defined through the following Lagrangian

$$
\mathcal{L}=B_{n-2} F, \quad B_{n-2}=\frac{1}{(n-2) !} d x^{\mu_{1}} \ldots d x^{\mu_{n-2}} B_{\mu_{1} \ldots \mu_{n-2}}, \quad F=\frac{1}{2} d x^{\mu} d x^{\nu} \partial_{\mu} A_{\nu}
$$


Evidently, this defines for $n>3$ a reducible gauge theory of reducibility order $r=n-3$ and implies that the abelian characteristic class $F$ vanishes on-shell. Hence, (8.10) does not hold in general for reducible gauge theories.

Contrary to the characteristic classes of form degrees $<n-1$, those of form degrees $(n-1)$ or $n$ can be trivial in the equivariant characteristic cohomology even in irreducible gauge theories. Part (ii) of theorem 7.1 shows that this can happen in the case $(n-1)$ only in the academical context that "free" abelian gauge fields are present. Indeed, the abelian Chern-Simons theory given by (8.13) for $n=3$ provides an example of this phenomenon. That characteristic classes of form degree $n$ can be trivial in the equivariant characteristic cohomology is also illustrated by (8.13), for $n=2$. A similar example in four dimensions is discussed at the end of section 9 .

\section{$9 \quad$ Result for $H^{*, n}(s \mid d)$}

According to section 6 it is now straightforward to construct $H^{*, *}(s \mid d)$. We only spell out $H^{*, n}(s \mid d)$ in some detail since it contains the solutions of direct physical interest. The discussion of these solutions is relegated to section 10. We will assume that (7.33) holds. At the end of the section we will illustrate that $(7.33)$ is a physically reasonable assumption and indicate the changes in the results if it is not satisfied.

The solutions $a^{g, n}$ of (1.1) arise via the descent equations from the functions (6.12) with ghost number $(g+n)$ according to section 6. One can distinguish three classes of them, stemming solely from $\mathcal{A}_{2}$, solely from $\mathcal{A}_{1}$, or from functions (6.12) involving only non-constant representatives of $H^{*}\left(s, \mathcal{A}_{i}\right)$.

Those of the first class stem from the cohomology classes of $H^{*}\left(s, \mathcal{A}_{2}\right)$. If "free" abelian gauge fields are absent and assumption (7.33) holds, this gives three different types of solutions $a_{i}^{g, n}(i=1,2,3)$ of $(1.1)$ arising from corresponding polynomials occurring in (7.34) respectively,

$$
\begin{array}{cl}
\hat{\Theta} L_{\tau}(\mathcal{T}) P^{\tau}(\theta(\hat{C})) & \rightarrow a_{1}^{g, n} \\
P(q, f) & \rightarrow a_{2}^{g, n} \\
G_{\Delta}^{*} P^{\Delta}(\theta(\hat{C})) & \rightarrow a_{3}^{g, n} .
\end{array}
$$

The solutions $a_{1}^{g, n}$ and $a_{2}^{g, n}$ do not involve antifields at all and are well-known in the literature. They have been denoted by $\mathcal{A}_{\text {trace }}$ and $\mathcal{A}_{\text {chiral }}$ respectively in [7] and are given there explicitly in eq. (7.42). We do not spell them out 
again, but just note that among them there are the invariant actions, as well as the chiral anomalies.

The solutions $a_{3}^{g, n}$ involve non-trivially the antifields and were previously unknown. According to section 6 they are given by the $n$-forms contained in $G_{\Delta}^{*} P^{\Delta}(\theta(\hat{C}))$ after replacing $\xi^{\mu}$ by $d x^{\mu}$. When doing so, one must not forget that the ghosts $\hat{C}^{I}$ involve the $\xi^{\mu}$ due to 3.2 ) which gives

$$
P(\theta(\hat{C}))=\left(1+\xi^{\mu} A_{\mu}{ }^{I} \frac{\partial}{\partial C^{I}}+\ldots\right) P(\theta(C))
$$

where the non-written terms are at least quadratic in the $\xi^{\mu}$. Using (7.7), we get

$$
a_{3}^{g, n}=d^{n} x e\left(X_{\Delta}^{*}+(-)^{\varepsilon_{\Delta}} J_{\Delta}^{\mu} A_{\mu}{ }^{I} \frac{\partial}{\partial C^{I}}\right) P^{\Delta, g+1}(\theta(C))
$$

with $J_{\Delta}^{\mu}=E_{a}{ }^{\mu} J_{\Delta}^{a}$. The polynomials $P^{\Delta, g+1}(\theta(C))$ have ghost number $(g+$ 1) since $a_{3}^{g, n}$ is supposed to have ghost number $g$. The factor $e$ in (9.2) originates from the substitution $\xi^{\mu} \rightarrow d x^{\mu}$ since the latter results in $\hat{\Theta} \rightarrow$ $d^{n} x e$. The different signs in front of the $J$-terms in (9.2) and (7.7) stem from the grading of the volume element. Note that in the important cases $g=0,1$ the polynomials $P^{\Delta, g+1}(\theta(C))$ have ghost number 1 and 2 respectively and therefore involve only abelian ghosts because the $\theta$ 's of the semi-simple parts of $\mathcal{G}$ have ghost numbers $\geq 3$. Hence, there are no solutions $a_{3}^{g, n}$ with $g=0,1$ if $G_{Y M}$ is semi-simple (contrary to other values of $g$ ).

If "free" abelian gauge fields are present, then the first class of solutions contains additional solutions of a fourth type. They stem from cohomology classes of $H^{*}\left(s, \mathcal{A}_{2}\right)$ whose representatives involve the $q_{\alpha}^{*}$ and therefore depend on antifields, cf. end of subsection 7.2. Their explicit form depends on the particular action. For the standard Lagrangian (2.11), one obtains from (7.14) and (7.53)

$$
a_{4}^{g, n} \stackrel{(2.11)}{=} d^{n} x \sum_{\alpha}\left(C_{\alpha}^{*}+A_{\alpha}^{* \mu} A_{\mu}^{I} \frac{\partial}{\partial C^{I}}+\frac{e}{2} F_{\alpha}^{\mu \nu} \mathcal{Z}_{\nu \mu}(C, A, F)\right) \frac{\partial P^{g+3}(\theta(C))}{\partial C^{\alpha}}
$$

where the $P^{g+3}(\theta(C))$ have ghost number $(g+3)$ and depend on the $\theta$ 's of the semi-simple part of $\mathcal{G}$ and on the "free" abelian ghosts $C^{\alpha}$, but not on other abelian ghosts. The $\mathcal{Z}_{\mu \nu}$ are field dependent operators

$$
\mathcal{Z}_{\mu \nu}(C, A, F)=A_{\mu}{ }^{J} A_{\nu}{ }^{I} \frac{\partial}{\partial C^{I}} \frac{\partial}{\partial C^{J}}+F_{\mu \nu}{ }^{I} q_{I,(K)}(C) \frac{\partial}{\partial \theta_{(K)}(C)}
$$


with

$$
q_{I,(K)}(C)=(-)^{m} \frac{m !(m-1) !}{(2 m-2) !} \operatorname{tr}\left(T_{I}^{(K)} \mathcal{C}^{2 m-3}\right), \quad m=m_{(K)}, \quad \mathcal{C}=C^{I} T_{I}^{(K)} .
$$

$\left[q_{I,(K)}(C)\right.$ vanishes for $\left.m_{(K)}=1\right]$. We note that $(9.3)$ agrees in fact with equation (8.17) of [34] up to the factor $e$ in front of the $F_{\alpha}^{\mu \nu}$-terms. The total antisymmetry of the coefficients $f$ occurring in equation (8.17) of [34 (in their $\alpha$-indices) is provided in (9.3) by the differentiation of $P^{g+3}$ with respect to $C^{\alpha}$ (and by the odd grading of the abelian ghosts contributing to $\left.P^{g+3}\right)$.

The solutions of the second class arise from $H^{*}\left(s, \mathcal{A}_{1}\right)$ and are denoted by $a_{5}^{g, n}$. They are simply the forms $\alpha_{m}^{k_{m}-n, n}$ corresponding to $\alpha_{m}^{k_{m}, 0}$ via the descent equations (cf. section 5),

$$
a_{5}^{g, n}=\sum_{\left\{m \mid k_{m}=n+g\right\}} \lambda_{m} \alpha_{m}^{k_{m}-n, n}
$$

where $\lambda_{m}$ are constants. Note that these solutions are present only for particular values of $g$ and $n$ since $k_{m}$ takes only special values obtained from the Lie algebra cohomology of $s o(n)$, cf. section 4 and appendix B.

The solutions of the third class arise from functions (6.12) involving only non-constant $\alpha_{m}^{k_{m}, 0}$ 's and $P_{A}$ 's. We denote them by $a_{6}^{g, n}$. The non-constant $P_{A}$ have ghost numbers $\geq n-2$ according to appendix $\mathrm{E}$ (lemma E.2). Since the non-constant $\alpha_{m}^{k_{m}, 0}$ have ghost number $k_{m} \geq 3$, we conclude that solutions $a_{6}^{g, n}$ exist only for $g \geq 1$. Of direct physical interest are only those with $g=1$ since they are candidate anomalies. They arise solely from the $\alpha_{m}^{k_{m}}$ with $k_{m}=3$ and the $P_{A}$ with ghost number $(n-2)$. The latter are just the $q_{\alpha}^{*}$, cf. lemma E.2. We get

$$
a_{6}^{1, n}=\sum_{\beta} \sum_{\left\{m \mid k_{m}=3\right\}} \sum_{i=0}^{3} \lambda_{m, \beta} \alpha_{m}^{k_{m}-i, i} a_{\beta}^{i-2, n-i}
$$

where $\lambda_{m, \beta}$ are constants and the $a_{\beta}^{i-2, n-i}$ are the $(n-i)$-forms occurring in the descent equations corresponding to the $q_{\beta}^{*}$, i.e.

$$
a_{\beta}^{-2, n}=d^{n} x C_{\beta}^{*}, \quad \cdots \quad, \quad a_{\beta}^{n-2,0}=q_{\beta}^{*} .
$$


Note that the solutions $a_{4}^{g, n}$ and $a_{6}^{1, n}$ are present only in the academical case that there are "free" abelian gauge fields. In particular there are no such solutions if $G_{Y M}$ is semi-simple.

Let us finally indicate modifications in the results if (7.33) is not valid. To illustrate them we consider a four dimensional theory whose Lagrangian has the standard form (2.11) up to the following extra piece:

$$
n=4: \quad \mathcal{L}^{\varphi} / e=\frac{1}{2} g^{\mu \nu}\left(\partial_{\mu} \varphi\right)\left(\partial_{\nu} \varphi\right)-\frac{1}{4} \varphi \varepsilon^{\mu \nu \rho \sigma} F_{\mu \nu}{ }^{I} F_{\rho \sigma}{ }^{J} d_{I J} .
$$

Here $\varphi$ is a real uncharged scalar field which for simplicity is assumed not to occur in the original Lagrangian (2.11). We note that the sum of (9.8) and (2.11) still defines a normal theory in the terminology of [14]. One easily verifies that (9.8) implies

$$
F^{I} F^{J} d_{I J} \approx-d b_{i n v}^{3}, \quad b_{i n v}^{3}=\frac{1}{3 !} d x^{\mu_{1}} \ldots d x^{\mu_{3}} \varepsilon_{\mu_{1} \ldots \mu_{4}} g^{\mu_{4} \nu} \partial_{\nu} \varphi
$$

which shows that assumption (7.33) is not valid in this case (as the reader may verify, $b_{i n v}^{3}$ can indeed be cast in the form (8.4) and therefore (9.9) is equivalent to $\hat{F}^{I} \hat{F}^{J} d_{I J} \approx-s_{1} \beta$, with $\beta \in \mathcal{A}_{2}$ ).

The physically most important changes in the results if (7.33) is not satisfied are:

(a) Some BRST invariant polynomials $P(q, f)$ which are non-trivial if (7.33) holds become trivial. In particular this can modify the results on chiral anomalies since some of them can become trivial. In the above four-dimensional example this happens to the abelian chiral candidate anomalies given by $C^{\text {abel }} F^{I} F^{J} d_{I J}$ where $C^{\text {abel }}$ is an abelian ghost. Namely (9.9) implies

$$
C^{a b e l} F^{I} F^{J} d_{I J}=s b^{0,4}+d b^{1,3}
$$

where

$$
b^{0,4}=A^{a b e l} b_{i n v}^{3}+d^{4} x C^{a b e l} \varphi^{*}, \quad b^{1,3}=C^{a b e l} b_{i n v}^{3}-i_{\xi} b^{0,4} .
$$

Here $A^{a b e l}=d x^{\mu} A_{\mu}{ }^{a b e l}$ is the abelian connection 1-form corresponding to $C^{\text {abel }}, b_{i n v}^{3}$ denotes the 3 -form given in (9.9), $\varphi^{*}$ is the antifield of $\varphi$ and $i_{\xi} b^{0,4}$ is the interior product (contraction) of the diffeomorphism ghost with the 4 -form $b^{0,4}$ (explicitly one has $i_{\xi}=\xi^{\mu} \partial / \partial\left(d x^{\mu}\right)$ on local forms). 
(b) Any characteristic class of form degree $n$ which is trivial in the equivariant characteristic cohomology corresponds to a conserved Noether current which, when written as an $(n-1)$-form, contains a Chern-Simons $(2 k-1)$-form. Indeed, $P^{2 k}(F):=d_{I_{1} \ldots I_{k}} F^{I_{1}} \ldots F^{I_{k}} \approx d b_{i n v}^{2 k-1}$ implies that $a^{2 k-1}=q^{2 k-1}(A, F)-b_{i n v}^{2 k-1}$ is weakly closed $\left(d a^{2 k-1} \approx 0\right)$ where $q^{2 k-1}(A, F)$ denotes the Chern-Simons form whose exterior derivative equals $P^{2 k}(F)$. Hence, $a^{2 k-1}$ indeed represents a conserved Noether current in $n=2 k$ dimensions. In the above four-dimensional example the conserved Noether current arising from (9.9) is given by

$$
j^{\mu}=e g^{\mu \nu} \partial_{\nu} \varphi+e \varepsilon^{\mu \nu \rho \sigma} d_{I J}\left(A_{\nu}{ }^{I} \partial_{\rho} A_{\sigma}{ }^{J}+\frac{1}{3} f_{K L}{ }^{J} A_{\nu}{ }^{I} A_{\rho}{ }^{K} A_{\sigma}{ }^{L}\right)
$$

and corresponds to the global symmetry of the action under $\varphi \rightarrow \varphi+$ constant. The latter is represented in $H^{-1, n}(s \mid d)$ simply by the volume form $d^{n} x \varphi^{*}$ (the non-trivial global symmetries and Noether currents correspond one-to-one to the cohomology classes of $H^{-1, n}(s \mid d)$, cf. [14 and section 10).

The above example illustrates also that the assumption (7.33) is physically reasonable since couplings of the type occurring in (9.8) are not present in the standard Einstein-Yang-Mills Lagrangian (2.11).

\section{Interpretation and discussion of the re- sults}

\subsection{Characteristic cohomology}

We first summarize the implications of our results for the characteristic cohomology, i.e. for the weak cohomology of $d$ in the space of local $p$-forms which depend neither on the ghosts nor on antifields $(p<n)$. As we have anticipated in the introduction, there are two types of cohomology classes, denoted by (i.a) and (i.b) respectively. Those of the type (i.a) are represented by the forms $\alpha_{m}^{0, k_{m}}$ introduced and discussed in section 4 . They are closed even offshell but fail to be exact because of the non-trivial De Rham cohomology of the vielbein manifold. Therefore we call them topological conserved $p$-forms.

The cohomology classes of type (i.b) are represented by local $p$-forms $a^{p}$ that are only weakly closed and not weakly exact. They are therefore called 
dynamically conserved $p$-forms and can be obtained from the cohomological groups $H^{p-n, n}(s \mid d)$. Indeed, $d a^{p} \approx 0$ is equivalent to $d a^{p}=-\delta \tilde{a}^{p+1}$ which implies $d a^{p}=-s a^{p+1}$ for some $(p+1)$-form $a^{p+1}=\tilde{a}^{p+1}+\ldots$ with ghost number -1 , cf. 35, 14. Note that $a^{p+1}$ vanishes only if $a^{p}$ is a topological conserved $p$-form up to a $d$-exact form. It is then straightforward, using the arguments of section 6, to show that $a^{p}$ corresponds via the descent equations indeed to a representative of a non-trivial cohomology class of $H^{p-n, n}(s \mid d)$, unless it is a topological conserved $p$-form up to a $d$-exact form?

The results of section 9 show that $H^{g, n}(s \mid d)$ is empty for $g<-2$. This implies that any weakly closed $p$-form is weakly exact for $p<n-2$, up to a topological conserved $p$-form. The cohomology classes of $H^{-2, n}(s \mid d)$ are represented by the solutions $a_{4}^{-2, n}$ in the notation of section 9 and are simply given by the volume forms $d^{n} x C_{\alpha}^{*}$, independently of the particular action. In contrast, the explicit form of the corresponding dynamically conserved $(n-2)$-forms depends on the chosen action. For the standard Lagrangian (2.11) they are simply given by

$$
a_{\alpha}^{n-2} \stackrel{(2.11)}{=} \frac{1}{2(n-2) !}(-)^{n+1} d x^{\mu_{1}} \ldots d x^{\mu_{n-2}} \varepsilon_{\mu_{1} \ldots \mu_{n}} F_{\alpha}^{\mu_{n-1} \mu_{n}} .
$$

Finally the dynamically conserved $(n-1)$-forms are just provided by the non-trivial Noether currents $\$$, up to topological conserved $(n-1)$-forms. In fact there are only two types of cohomology classes of $H^{-1, n}(s \mid d)$ if $(7.33)$ holds. Their representatives have been denoted in section 9 by $a_{3}^{-1, n}$ and $a_{4}^{-1, n}$ respectively. The latter occur only if "free" abelian gauge fields are present. The former correspond bijectively to the functions $G_{\Delta}^{*}$ introduced in section 7.1 and are given explicitly by

$$
a_{\Delta}^{n-1}=\frac{1}{(n-1) !} d x^{\mu_{1}} \ldots d x^{\mu_{n-1}} \varepsilon_{\mu_{1} \ldots \mu_{n}} E_{a}^{\mu_{n}} J_{\Delta}^{a}(\mathcal{T}) .
$$

The corresponding Noether currents satisfying $\partial_{\mu} j_{\Delta}^{\mu} \approx 0$ are given by

$$
j_{\Delta}^{\mu}=e E_{a}^{\mu} J_{\Delta}^{a}(\mathcal{T})
$$

\footnotetext{
${ }^{7}$ Note: these descent equations go down to a zero-form according to section 6 and thus $a^{p}$ is just the $p$-form with ghost number 0 occurring in them.

${ }^{8}$ In this context a Noether current is called trivial if it reduces on-shell to an identically conserved current of the form $\partial_{\nu} S^{\nu \mu}$ with $S^{\nu \mu}=-S^{\mu \nu}$. A global symmetry is called trivial if it reduces on-shell to a gauge transformation with field dependent parameters, cf. [14] for details.
} 
To summarize, we obtain almost the same result for the characteristic cohomology as in [14]. The only difference is the occurrence of the topological conserved $p$-forms. The reason why the non-contractibility of the vielbein manifold does not lead to further modifications may be seen from the proof of lemma E.2.

The results on the equivariant characteristic cohomology have been summarized already in section 8 and are not repeated here.

\subsection{Covariance properties of the Noether currents and of the global symmetries}

The results of subsection 10.1 allow us to conclude that all non-trivial Noether currents can be covariantized if "free" abelian gauge fields are absent and assumption (7.33) holds. Indeed, we have seen that then all non-trivial Noether currents can be assumed to be of the form (10.1). The latter are invariant under local Lorentz and Yang-Mills gauge transformations and transform as contravariant vector densitites under general coordinate transformations due to (7.6), i.e. they satisfy

$$
s j_{\Delta}^{\mu}=\partial_{\nu}\left(\xi^{\nu} j_{\Delta}^{\mu}\right)-\left(\partial_{\nu} \xi^{\mu}\right) j_{\Delta}^{\nu} .
$$

The corresponding (infinitesimal) global symmetries of the action are encoded in the part $X_{\Delta}^{*}$ of $G_{\Delta}^{*}$. Recall that the $X_{\Delta}^{*}$ are defined only up to functions of the form $\delta M\left(\mathcal{T}, \mathcal{T}^{*}\right)+D_{a} N^{a}\left(\mathcal{T}, \mathcal{T}^{*}\right)$. We can therefore remove all covariant derivatives acting on the antifields by subtracting suitable parts $D_{a} N^{a}$ from $X_{\Delta}^{*}$ and assume with no loss of generality that

$$
X_{\Delta}^{*}=\hat{y}_{m}^{*} X_{\Delta}^{m}(\mathcal{T})+\hat{e}_{a}^{* b} X_{\Delta b}^{a}(\mathcal{T})+\hat{A}_{i}^{* a} X_{\Delta a}^{i}(\mathcal{T})
$$

The global symmetries of the classical action, denoted by $\delta_{\Delta}$, are then just given by

$$
\delta_{\Delta} y^{m}=X_{\Delta}^{m}(\mathcal{T}), \quad \delta_{\Delta} e_{\mu}{ }^{a}=e_{\mu}{ }^{b} X_{\Delta b}^{a}(\mathcal{T}), \quad \delta_{\Delta} A_{\mu}{ }^{i}=e_{\mu}{ }^{a} X_{\Delta a}^{i}(\mathcal{T})
$$

Due to $\delta_{I} X_{\Delta}^{*}=0$ these transformation are gauge-covariant in the sense that $\left(\phi+\delta_{\Delta} \phi\right)$ transforms exactly as $\phi$ under diffeomorphisms, local Lorentz and Yang-Mills transformations. Together with remark (b) at the end of section 9 we conclude: 
Theorem 10.1 If "free" abelian gauge fields are absent and (7.33) holds, then

(a) all non-trivial global symmetries can be chosen to be gauge-covariant, i.e. to be of the form (10.4) where the covariance properties of the $X$ 's are indicated by their indices $m, a, b, i$;

(b) all non-trivial Noether currents can be chosen to be gauge invariant under Lorentz and Yang-Mills transformations and to transform as contravariant vector-densities under diffeomorphisms. More precisely, they are of the form (10.1) and satisfy (7.0) resp. (10.9).

If (7.33) does not hold, then there are non-trivial Noether currents which can be chosen to be of the form (b) only up to Chern-Simons terms. These Noether currents correspond one-to-one to those characteristic classes of form degree $n$ which become trivial in the equivariant characteristic cohomology.

We finally remark that (a) and (b) do not always hold in presence of "free" abelian gauge fields. This is easily verified for the standard Lagrangian (2.11) if the latter contains more than one "free" abelian gauge field. Namely then (2.11) has the global symmetry $\delta_{\epsilon} A_{\mu}{ }^{\alpha}=\epsilon_{\alpha \beta} A_{\mu}{ }^{\beta}$ where $\epsilon_{\alpha \beta}=-\epsilon_{\beta \alpha}$ are constants $\left(\delta_{\epsilon}\right.$ vanishes on all other fields). Evidently $\delta_{\epsilon}$ is not gauge-covariant in the above sense (and cannot be covariantized) and one easily checks that the corresponding Noether current does not satisfy (10.2) (the corresponding cohomology class of $H^{-1, n}(s \mid d)$ is represented by (9.3), choosing $P^{2}(\theta(C))=$ $\frac{1}{2} \varepsilon_{\alpha \beta} C^{\alpha} C^{\beta}$ there).

Theorem 10.1 is analogous to the result we have obtained in [36] for YangMills theories (in flat spacetime). Note however that in the gravitational case all non-trivial global symmetries and Noether currents can be chosen such that they do not depend explicitly on the spacetime coordinates $x^{\mu}$, contrary to the results in flat spacetime.

\subsection{Consistent deformations of the action}

The BRST cohomology on local functionals with ghost number 0 provides the possible consistent deformations of the action and its gauge symmetries. A systematic construction of such deformations has been described in [ [One looks for a functional with ghost number 0 of the form

$$
\mathcal{S}^{e x t}=\mathcal{S}^{(0)}+g \mathcal{S}^{(1)}+g^{2} \mathcal{S}^{(2)}+\ldots
$$


satisfying the master equation,

$$
\left(\mathcal{S}^{e x t}, \mathcal{S}^{e x t}\right)=0
$$

Here $\mathcal{S}^{(0)}$ denotes the original solution of the classical master equation and $g$ is a "coupling constant". Expanding (10.6) in powers of $g$ one finds that the $\mathcal{S}^{(k)}$ have to satisfy

$$
\left(\mathcal{S}^{(0)}, \mathcal{S}^{(0)}\right)=0, \quad\left(\mathcal{S}^{(0)}, \mathcal{S}^{(1)}\right)=0, \quad\left(\mathcal{S}^{(1)}, \mathcal{S}^{(1)}\right)+2\left(\mathcal{S}^{(0)}, \mathcal{S}^{(2)}\right)=0, \quad \ldots
$$

where the first equation is just the original master equation and thus satisfied by assumption. The second equation requires $\mathcal{S}^{(1)}$ to be invariant under the original BRST operator $s$ whereas the third condition requires $\left(\mathcal{S}^{(1)}, \mathcal{S}^{(1)}\right)$ to be $s$-exact. Contributions to $\mathcal{S}^{(1)}$ which are already $s$-exact can be neglected since they can be absorbed by field redefinitions [4].

Now, one can distinguish two types of deformations, denoted by (ii.a) and (ii.b) respectively. Those of type (ii.a) modify only the classical action but not the form of its gauge symmetries. They correspond to antifield independent local functionals $\mathcal{S}^{(1)}$ which are BRST closed. For these, there is no higher order obstruction to the deformation since $\left(\mathcal{S}^{(1)}, \mathcal{S}^{(1)}\right)$ evidently vanishes. Thus, one can set $\mathcal{S}^{(i)}=0$ for $i>1$. Deformations of the type (ii.b) modify simultaneously the classical action and the form (and, in general, the algebra) of its gauge symmetries. They correspond to solutions $\mathcal{S}^{(1)}$ of the first order condition $\left(\mathcal{S}^{(0)}, \mathcal{S}^{(1)}\right)=0$ that depend non-trivially on the antifields. Such first order deformation may be obstructed at higher order, e.g. if $\left(\mathcal{S}^{(1)}, \mathcal{S}^{(1)}\right)$ is not BRST exact in the space of local functionals. These higher order obstructions will not be discussed here.

We have seen above that $\mathcal{S}^{(1)}$ is non-trivially $s$-invariant. Hence, it arises from the cohomology classes of $H^{0, n}(s \mid d)$. The results of section 9 show that the latter are represented by volume forms which do not depend on antifields at all, unless the Yang-Mills gauge group contains abelian factors. Indeed, the only antifield dependent ghost number 0-solutions occurring in section 9 are the $a_{3}^{0, n}$ and $a_{4}^{0, n}$ and all of them involve abelian factors of $G_{Y M}$. We conclude that deformations of the type (ii.b) do not exist if $G_{Y M}$ is semisimple, and that the only possible deformations are in that case obtained simply by adding further gauge invariant pieces to the action (involving e.g. higher powers in the curvatures or higher order derivatives). 
If abelian factors are present, there are in general also deformations of type (ii.b). Their precise form depends on the original classical action and its global symmetries. We just note that among these deformations there are those whose part $\mathcal{S}^{(1)}$ includes the Yang-Mills cubic vertex for a set of originally non-interacting abelian gauge fields and the non-abelian extension of their gauge transformations, cf. [4] $\left(\mathcal{S}^{(2)}\right.$ then provides the Yang-Mills quartic vertex).

\subsection{Candidate anomalies}

The candidate anomalies correspond to the representatives of the cohomology classes of $H^{1, n}(s \mid d)$. We distinguish three types of them, denoted by (iii.a), (iii.b) and (iii.c).

Those of type (iii.a) are given by the solutions (9.6) and (9.7) with ghost number 1 . Since they stem from the non-trivial De Rham cohomology of the vielbein manifold, we call them topological candidate anomalies. Solutions (9.6) with ghost number 1 exist only for $n=n_{0}+4 k$ with $n_{0}=6,9,20,35$ $(k=0,1, \ldots)$ as follows from the Lie algebra cohomology of $s o(n)$, whereas the solutions (9.7) occur only in presence of "free" abelian gauge fields. An interpretation of the topological candidate anomalies is not known to us.

The candidate anomalies of type (iii.b) and (iii.c) arise solely from cohomology classes of $\mathcal{A}_{2}$ with ghost number $(n+1)$. Those of type (iii.b) do not involve the antifields. They have been classified already in [7] where however antifield dependent counterterms which can cancel these candidate anomalies have not been considered. Following [7], to which we refer for a thorough analysis, there are two types of candidate anomalies (iii.b), those of the "trace type" and the "chiral anomalies" which in our notation are the solutions $a_{1}^{1, n}$ and $a_{2}^{1, n}$ respectively, cf. section 9. The former are present only if the gauge group contains abelian factors. Our results show that all chiral candidate anomalies given in [7] remain non-trivial even if one includes antifield dependent counterterms, at least if (7.33) is satisfied (remark (a) at the end of section 9 shows that some chiral candidate anomalies can become trivial if (7.33) is not valid).

The candidate anomalies (iii.c) depend non-trivially on the antifields. They are denoted by $a_{3}^{1, n}$ and $a_{4}^{1, n}$ in section 9. Again, these solutions occur only if the gauge group contains abelian factors.

We conclude that the well-known chiral anomalies are the only candi- 
date anomalies if the Yang-Mills gauge group is semi-simple, apart from the topological candidate anomalies occurring in $n=6,9,10,13, \ldots$ spacetime dimensions.

\section{Acknowledgements}

This work has been supported in part by research funds from F.N.R.S., by a research contract with the Commission of the European Communities, and by the research council (DOC) of the K.U. Leuven.

\section{A Conventions}

Minkowski metric, $\varepsilon$-tensor, volume forms:

$$
\begin{aligned}
& \eta_{a b}=\operatorname{diag}(1,-1, \ldots,-1), \\
& \varepsilon^{a_{1} \ldots a_{n}}=\varepsilon^{\left[a_{1} \ldots a_{n}\right]}, \quad \varepsilon^{0 \ldots(n-1)}=1, \quad \varepsilon_{\mu_{1} \ldots \mu_{n}}=\varepsilon_{a_{1} \ldots a_{n}} e_{\mu_{1}}{ }^{a_{1}} \ldots e_{\mu_{n}}{ }^{a_{n}}, \\
& d^{n} x=d x^{0} \ldots d x^{n-1}=\frac{(-)^{n+1}}{n !} d x^{\mu_{1}} \ldots d x^{\mu_{n}} \varepsilon_{\mu_{1} \ldots \mu_{n}} / e .
\end{aligned}
$$

(Anti-) Symmetrization of indices, symmetrized trace of matrices:

$$
\begin{aligned}
T_{\left(a_{1} \ldots a_{k}\right)} & =\frac{1}{k !} \sum_{\pi} T_{a_{\pi(1)} \ldots a_{\pi(k)}}, \\
T_{\left[a_{1} \ldots a_{k}\right]} & =\frac{1}{k !} \sum_{\pi}(-)^{\operatorname{sign}(\pi)} T_{a_{\pi(1)} \ldots a_{\pi(k)}}, \\
\operatorname{Str}\left(M_{1} \ldots M_{k}\right) & =\frac{1}{k !} \sum_{\pi} \operatorname{tr}\left(M_{\pi(1)} \ldots M_{\pi(k)}\right)
\end{aligned}
$$

where $\sum_{\pi}$ runs over all permutations $\pi$ in the symmetric group $S_{k}$.

Antibracket:

$$
(X, Y)=\int d^{n} x\left(\frac{\delta^{R} X}{\delta \Phi^{A}(x)} \frac{\delta^{L} Y}{\delta \Phi_{A}^{*}(x)}-\frac{\delta^{R} X}{\delta \Phi_{A}^{*}(x)} \frac{\delta^{L} Y}{\delta \Phi^{A}(x)}\right)
$$

where $\delta^{R} / \delta Z(x)$ and $\delta^{L} / \delta Z(x)$ denote functional right- and left-derivatives. The BRST operator $s$ is defined by means of the antibracket with the proper solution $\mathcal{S}$ of the classical master equation through

$$
s X=(\mathcal{S}, X) \text {. }
$$




\section{B The Lie algebra cohomology of $\mathcal{G}$ in $\mathcal{A}_{2}$}

The Lie algebra cohomology of $\mathcal{G}$ in $\mathcal{A}_{2}$ can be described in terms of its primitive elements (7.1) and the functions in $\mathcal{A}_{2,0}^{\text {inv }}$ defined in (B.3) [37]:

$$
\begin{aligned}
& \gamma_{\mathcal{G}} \alpha=0, \alpha \in \mathcal{A}_{2} \quad \Leftrightarrow \quad \alpha=\alpha^{i} P_{i}(\theta(\hat{C}))+\gamma_{\mathcal{G}} \beta, \alpha^{i} \in \mathcal{A}_{2,0}^{i n v} \\
& \alpha^{i} P_{i}(\theta(\hat{C}))=\gamma_{\mathcal{G}} \beta, \quad \alpha^{i} \in \mathcal{A}_{2,0}^{i n v} \quad \Leftrightarrow \quad \alpha^{i}=0 \quad \forall i
\end{aligned}
$$

where $\beta \in \mathcal{A}_{2}$ and the $P_{i}(\theta)$ are linearly independent polynomials in the $\theta_{(K)}$ and $\mathcal{A}_{2,0}^{\text {inv }}$ is the subspace of $\mathcal{A}_{2}$ containing the $\delta_{I}$-invariant functions which do not depend on the ghosts $\hat{C}^{I}$,

$$
\mathcal{A}_{2,0}^{i n v}=\left\{\alpha \in \mathcal{A}_{2}: \frac{\partial \alpha}{\partial \hat{C}^{I}}=\delta_{I} \alpha=0\right\} .
$$

The Lie algebra cohomology of $s o(n-k, k)$ has in $n=2 r$ and $n=2 r+1$ dimensions $r$ primitive (real) elements given by

$$
\begin{aligned}
\theta_{(K)}(C) & =N_{(K)} C_{a_{1}}^{a_{2}} B_{a_{2}}^{a_{3}} \ldots B_{a_{2(K)}{ }^{a_{1}}, \quad(K)=1,2, \ldots, r-1,} \\
\theta_{r}(C) & =N_{r} \begin{cases}C_{a_{1}}^{a_{2}} B_{a_{2}}{ }^{a_{3}} \ldots B_{a_{2 r} a_{1}} & \text { for } n=2 r+1 \\
\varepsilon_{a_{1} b_{1} \ldots a_{r} b_{r}} C^{a_{1} b_{1}} B^{a_{2} b_{2}} \ldots B^{a_{r} b_{r}} & \text { for } n=2 r\end{cases}
\end{aligned}
$$

where $B_{a}{ }^{b}=C_{a}{ }^{c} C_{c}{ }^{b}$. These $\theta_{(K)}$ agree for proper normalization factors $N_{(K)}$ with (7.1), using $m_{(K)}=2(K)$ and choosing for $\left\{T_{a b}^{(K)}\right\}$ the adjoint representation of $s o(n-k, k)$ except for the case $n=2 r,(K)=r$ in which one needs the spinor representation [30].

Analogously one constructs the $f_{(K)}$ for $s o(n-k, k)$ in the cases $n=2 r$ and $n=2 r+1$ :

$$
\begin{aligned}
f_{(K)} & =\hat{F}_{a_{1}}^{a_{2}} \hat{F}_{a_{2}}{ }^{a_{3}} \ldots \hat{F}_{a_{2(K)}{ }^{a_{1}},}, \quad(K)=1,2, \ldots, r-1, \\
f_{r} & = \begin{cases}\hat{F}_{a_{1}{ }^{a_{2}}} \hat{F}_{a_{2}}^{a_{3}} \ldots \hat{F}_{a_{2 r} a_{1}} \hat{a}_{1} & \text { for } n=2 r+1 \\
\hat{F}^{a_{1} b_{1}} \ldots \hat{F}^{a_{r} b_{r}} \varepsilon_{a_{1} b_{1} \ldots a_{r} b_{r}} & \text { for } n=2 r .\end{cases}
\end{aligned}
$$

Notice that $f_{(K)}$ vanishes for $(K)>n / 4$, except for $f_{r}$ in $n=2 r$ dimensions.

\section{BRST invariant subalgebras of $\mathcal{A}_{2}$}

Since $\mathcal{A}_{2}$ is a polynomial algebra and since $\mathcal{G}=\mathcal{G}_{L}+\mathcal{G}_{Y M}$ is reductive, we can uniquely decompose any function $\alpha \in \mathcal{A}_{2}$ into parts $\alpha^{\lambda}$ transforming under 
$G$ according to a particular irreducible representation $\lambda$. Hence, $\mathcal{A}_{2}$ decomposes into subalgebras $\mathcal{A}_{2}^{\lambda}$ containing only functions transforming under $G$ according to $\lambda$. Since $s$ commutes with all $\delta_{I}$ it leaves invariant each of these subalgebras, i.e. the image of $\mathcal{A}_{2}^{\lambda}$ under $s$ is contained in $\mathcal{A}_{2}^{\lambda}$,

$$
s\left[\mathcal{A}_{2}^{\lambda}\right] \subset \mathcal{A}_{2}^{\lambda} .
$$

This implies: (a) a function $\alpha \in \mathcal{A}_{2}$ is $s$-invariant iff all its parts $\alpha^{\lambda}$ are separately $s$-invariant and (b) $\alpha \in \mathcal{A}_{2}$ is $s$-exact in $\mathcal{A}_{2}$ iff the $\alpha^{\lambda}$ are $s$-exact in $\mathcal{A}_{2}^{\lambda}$ respectively,

$$
\begin{aligned}
& \text { (a) } \quad s \alpha=0, \quad \alpha \in \mathcal{A}_{2} \Leftrightarrow s \alpha^{\lambda}=0 \quad \forall \lambda ; \\
& \text { (b) } \alpha=s \beta, \quad \alpha, \beta \in \mathcal{A}_{2} \Leftrightarrow \alpha^{\lambda}=s \beta^{\lambda} \quad \forall \lambda .
\end{aligned}
$$

\section{Cohomology of $\delta$ in $\mathcal{A}_{2}$}

That $H_{k}(\delta, \mathcal{A})$ is zero for positive antighost number $k$ is a standard result and holds by construction of the Koszul-Tate complex, see e.g. 38. Since functions in $\mathcal{A}_{1}$ do not depend on antifields at all, the subspaces of $\mathcal{A}$ with non-vanishing antighost number agree with those of $\mathcal{A}_{2}$. Hence, $H_{k}\left(\delta, \mathcal{A}_{2}\right)$ is zero for $k>0$. According to appendix C, $\mathcal{A}_{2}$ can be decomposed into the subalgebras $\mathcal{A}_{2}^{\lambda}$. The latter can be further decomposed into subalgebras $\mathcal{A}_{2, q}^{\lambda}$ containing functions with $\hat{C}$-degree $q$. Now, $\delta$ leaves invariant each $\mathcal{A}_{2, q}^{\lambda}$ since it does not change the $\hat{C}$-degree and commutes with all $\delta_{I}$. By the same reasoning as in appendix $\mathrm{C}$, we conclude that $H_{k}\left(\delta, \mathcal{A}_{2, q}^{\lambda}\right)$ is zero in positive antighost number $k$ for all $q$ and $\lambda$ separately.

\section{E Proof of the weak covariant Poincaré lem- ma}

We first prove that the solution of $(7.29)$ and $(\sqrt{7.30})$ is equivalent to the computation of the BRST cohomology in the subspace $\mathcal{A}_{2,0}^{\text {inv }}$ of $\mathcal{A}_{2}$ defined in (B.3).

Lemma E.1 Any solution $\alpha_{\ell}^{i}(\hat{\xi}, \mathcal{T})$ of (7.29) and (7.30) with ghost number $\ell$ can be completed to a function $\alpha \in \mathcal{A}_{2,0}^{\text {inv }}$ representing a non-trivial cohomology class of $H^{\ell}\left(s, \mathcal{A}_{2,0}^{\text {inv }}\right)$. Conversely, any representative of a non-trivial 
cohomology class of $H^{\ell}\left(s, \mathcal{A}_{2,0}^{\text {inv }}\right)$ contains a solution $\alpha_{\ell}^{i}(\hat{\xi}, \mathcal{T})$ of (7.29) and (7.30).

Proof. To prove the lemma one takes advantage of the facts that the BRST operator reduces to $s_{1}+\delta$ in $\mathcal{A}_{2,0}^{i n v}$ and that $s_{1}$ is nilpotent in $\mathcal{A}_{2,0}^{i n v}$,

$$
\alpha \in \mathcal{A}_{2,0}^{i n v} \Rightarrow s_{1}^{2} \alpha=0, \quad s \alpha=\left(s_{1}+\delta\right) \alpha,
$$

which holds according to $(7.17)-(7.23)$ due to $\gamma_{\mathcal{G}}\left[\mathcal{A}_{2,0}^{\text {inv }}\right]=s_{2}\left[\mathcal{A}_{2,0}^{\text {inv }}\right]=0$. The proof is now straightforward using standard arguments of homological perturbation theory, cf. [38]. To see that any solution of (7.29) can be completed to a BRST invariant function $\alpha \in \mathcal{A}_{2,0}^{\text {inv }}$ one acts with $s_{1}$ on (7.29). Using (E.1) this yields $\delta\left(s_{1} \alpha_{\ell+1}^{i}\right)=0$ which implies $\delta \alpha_{\ell+2}^{i}+s_{1} \alpha_{\ell+1}^{i}=0$ for some $\alpha_{\ell+2}^{i} \in \mathcal{A}_{2,0}^{i n v}$ due to the acyclicity of $\delta$ in positive antighost number, cf. appendix D. Iterating the argument one concludes the existence of functions $\alpha_{p}^{i} \in \mathcal{A}_{2,0}^{i n v}$ with $\hat{\xi}$-degrees $p=\ell, \ell+1, \ldots, n$ such that $\alpha=\sum \alpha_{p}^{i}$ satisfies $\left(s_{1}+\delta\right) \alpha=0$, and thus $s \alpha=0$ according to (E.1). Using (E.1) and the acyclicity of $\delta$ in positive antighost number again, one shows analogously that $\alpha \in \mathcal{A}_{2,0}^{i n v}$ is BRST exact in $\mathcal{A}_{2,0}^{i n v}$ iff its antifield independent part is weakly $s_{1}$-exact.

Theorem 7.1 can therefore be proved by computing $H^{*}\left(s, \mathcal{A}_{2,0}^{\text {inv }}\right)$. To that end we will take advantage of results derived in [14] for the cohomology groups $H_{*}^{*}(\delta \mid d)$ which is the relative cohomology of $\delta$ modulo $d$ in the space of local forms. However we cannot directly adopt these results for the theories at hand since we have assumed in [14] the field-antifield manifold to be contractible to a point which fails to be true here because of the noncontractibility of the vielbein manifold, cf. section 4 . In particular the proof given in [14] for normal theories uses a perturbative argument which applies directly to gravitational theories only if one splits the vielbein into a constant background vielbein and a deviation $h_{\mu}^{a}$ from it. The proof given in [14] works then only in the space of forms which are polynomial in the derivatives of all fields, but involve in general formal infinite power series' in the undifferentiated $h_{\mu}^{a}$, without guaranteeing that these series' are really regular (smooth) in the $e_{\mu}^{a}$ (for $\operatorname{det}\left(e_{\mu}^{a}\right) \neq 0$ ). We will first show now that the series' can be really assumed to be regular in all cases we need. This is encoded in the following lemma, as becomes clear from its proof:

Lemma E.2 $H^{G}\left(s, \mathcal{A}_{2}\right)$ is zero for $G<0$ and $0<G<n-2, H^{0}\left(s, \mathcal{A}_{2}\right)$ contains only one non-trivial cohomology class represented by a constant, and 
the non-trivial cohomology classes of $H^{n-2}\left(s, \mathcal{A}_{2}\right)$ are represented by the $q_{\alpha}^{*}$,

$$
\begin{aligned}
& s \alpha=0, \quad \alpha \in \mathcal{A}_{2}, \quad g h(\alpha)=G \leq n-2 \\
& \quad \Leftrightarrow \quad \alpha=\delta_{G}^{n-2} \lambda^{\alpha} q_{\alpha}^{*}+\delta_{G}^{0} \lambda+s \beta, \quad \beta \in \mathcal{A}_{2}
\end{aligned}
$$

where $\lambda^{\alpha}$ and $\lambda$ are constants and $\delta_{G}^{p}$ denotes the Kronecker symbol $\left(\delta_{G}^{p}=1\right.$ if $G=p$ and $\delta_{G}^{p}=0$ if $G \neq p$ ).

Proof. Assume that $\alpha \in \mathcal{A}_{2}$ is BRST invariant and has ghost number $G<n-2$ (the case $G=n-2$ is considered separately below). According to theorem 6.1, it corresponds via the descent equations to a volume form $a^{G-n, n}$ solving (1.1) or it is BRST exact up to a constant. A constant can occur of course only in the case $G=0$. Note that $a^{G-n, n}$ has ghost number $G-n<-2$. General results on the local BRST cohomology derived first in [35] and refined for normal theories in [14, 34] state that $H^{g, n}(s \mid d)$ is isomorphic to $H_{-g}^{n}(\delta \mid d)$ for $g<0$, the latter being the cohomology of $\delta$ modulo $d$ in the space of local $n$-forms with antighost number $(-g)$.

Now, in [14] we have proved that the cohomology groups $H_{k}^{n}(\delta \mid d)$ vanish for $k>2$ in normal irreducible gauge theories treated there. Assume that the statement remains true even in our case for the volume forms $a^{G-n, n}$ which arise from BRST invariant functions $\alpha \in \mathcal{A}_{2}$. Then we could conclude that $a^{G-n, n}$ is trivial and thus that $\alpha$ is BRST exact up to a constant which would prove the theorem for $G<n-2$.

However, as mentioned above, the proof given in [14] applies to the gravitational theories in question directly only for forms that are formal infinite power series' in the undifferentiated $e_{\mu}{ }^{a}$ (but still polynomials in the derivatives of all fields, including $e_{\mu}{ }^{a}$ ). Let us call such forms "quasi-local". Hence, the results of 14 allow us only to conclude that $\alpha$ is $s$-exact in the space of quasi-local functions, i.e. that

$$
\alpha=s \beta+\delta_{G}^{0} \lambda
$$

holds for some quasi-local function $\beta$. We have to show now that we can even assume $\beta$ to be (strictly) local. This becomes obvious when one expresses $\beta$ in terms of the generators introduced in section 3 and decomposes it into parts $\beta^{(r)}$ with definite degree $r$ in the generators $U_{l}$ and $V_{l}$. Due to (3.8) and (3.20) the counting operator for all the $U$ 's and $V$ 's commutes with $s$. Therefore $(\mathbb{E} .3)$ requires $s \beta^{(r)}=0$ for all $r \neq 0$ and $s \beta^{(0)}=\alpha-\delta_{G}^{0} \lambda$ since $\alpha$ 
does not depend on the $U$ 's and $V$ 's at all due to $\alpha \in \mathcal{A}_{2}$. Note that $\beta^{(0)}$ is local since $\beta$ is quasi-local. We conclude $\beta^{(0)} \in \mathcal{A}_{2}$ which proves the lemma for $G<n-2$.

For $G=n-2$ the results of [14] (section 13) and equation (7.11) show that $a^{G-n, n}=a^{-2, n}$ is given by a linear combination of the $n$-forms $d^{n} x C_{\alpha}^{*}$, up to a piece which is trivial in the space of quasi-local forms. The former corresponds via the descent equations to a linear combination of the functions $q_{\alpha}^{*}$, cf. subsection 7.1, i.e. we conclude $\alpha-\lambda^{\alpha} q_{\alpha}^{*}=s \beta$ for some quasi-local function $\beta$. Due to $q_{\alpha}^{*} \in \mathcal{A}_{2}$ we can use now the same argument as above to conclude $\alpha-\lambda^{\alpha} q_{\alpha}^{*}=s \beta^{(0)}$ for $\beta^{(0)} \in \mathcal{A}_{2}$.

This completes the demonstration of the lemma and shows simultaneously that the results of [14] on $H_{k}^{p}(\delta \mid d)$ remain valid in our case for forms corresponding via the descent equations to BRST invariant functions $\alpha \in \mathcal{A}_{2}$ (and for $p \leq n+k-2$ ). Note that an analogous conclusion is in fact not true for $\alpha \in \mathcal{A}$. Indeed, the vielbein manifold enters through $\mathcal{A}_{1}$ and gives rise to the functions $\alpha_{m}^{k_{m}, 0}$ (and the forms $\alpha_{m}^{0, k_{m}}$ ) which represent non-trivial cohomology classes of $H^{k_{m}}(s)\left(H^{k_{m}}(d)\right)$ although they are $s$-exact (d-exact) in the space of quasi-local functions (forms). Note that this implies that any function (6.12) is $s$-exact in the space of quasi-local functions except for those which involve the constant representative of $H^{0}\left(s, \mathcal{A}_{1}\right)$.

It is important to realize that to compute $H^{*}\left(s, \mathcal{A}_{2,0}^{\text {inv }}\right)$ it is not sufficient to determine only those BRST invariant functions $\alpha \in \mathcal{A}_{2,0}^{\text {inv }}$ which are not BRST exact. Namely it can (and does) happen that a function $\alpha \in \mathcal{A}_{2,0}^{\text {inv }}$ is BRST exact in $\mathcal{A}_{2}$ but not in $\mathcal{A}_{2,0}^{\text {inv }}$. We shall determine first these functions by means of lemma E.2. To this end we assume that $\alpha \in \mathcal{A}_{2,0}^{\text {inv }}$ is BRST exact (in $\mathcal{A}_{2}$ ) and has ghost number $<n$,

$$
\alpha=s \beta, \quad \alpha \in \mathcal{A}_{2,0}^{i n v}, \quad \beta \in \mathcal{A}_{2}, \quad g h(\alpha)=G<n .
$$

We now differentiate (E.4) with respect to $\hat{C}^{I}$. Since $\alpha$ does not depend on $\hat{C}^{I}$, we obtain

$$
0=\left(s \hat{\partial}_{I}+\hat{\partial}_{I} s\right) \beta-s \hat{\partial}_{I} \beta
$$

where we have introduced the notation

$$
\hat{\partial}_{I}=\frac{\partial}{\partial C^{I}}
$$


We now take advantage of the fact that $s \hat{\partial}_{I}+\hat{\partial}_{I} s$ represents $\delta_{I}$ on $\mathcal{A}_{2}$, as follows immediately from (7.17)-(7.22):

$$
\delta_{I}=s \hat{\partial}_{I}+\hat{\partial}_{I} s .
$$

According to appendix $\mathbb{Q}$ we can assume $\delta_{I} \beta=0$ with no loss of generality due to $\delta_{I} \alpha=0$. Hence, we conclude from (E.5) and (E.7)

$$
s\left(\hat{\partial}_{I} \beta\right)=0 .
$$

For later use we note that the functions $\hat{\partial}_{I} \beta$ transform under the co-adjoint representation of $\mathcal{G}$ due to $\delta_{I} \beta=0$ :

$$
\delta_{I}\left(\hat{\partial}_{J} \beta\right)=f_{I J}^{K} \hat{\partial}_{K} \beta .
$$

This follows directly from $\delta_{I} \beta=0$ due to

$$
\left[\delta_{I}, \hat{\partial}_{J}\right]=f_{I J}{ }^{K} \hat{\partial}_{K}
$$

Note that the $\hat{\partial}_{I} \beta$ have ghost number $G-2<n-2$ due to $g h(\beta)=g h(\alpha)-1$. Therefore we can conclude from (E.8), using lemma E.2:

$$
\hat{\partial}_{I} \beta=d_{I} \delta_{G}^{2}+s \beta_{I}, \quad d_{I}=\text { const. }
$$

The Kronecker-symbol $\delta_{G}^{2}$ indicates that constants can contribute to $\hat{\partial}_{I} \beta$ only if the latter have vanishing ghost number. Recall that the $\hat{\partial}_{I} \beta$ transform according to $(\mathbb{E} .9)$ under $\mathcal{G}$. This implies that both $s \beta_{I}$ and $d_{I}$ can be assumed to transform under the co-adjoint representation of $\mathcal{G}$ since the decomposition $d_{I}+s \beta_{I}$ is unique, as the constants are not BRST exact?.

On the one hand this implies that $d_{I}$ can be different from zero only if its index $I$ refers to an abelian element of $\mathcal{G}$ since the semi-simple part of $\mathcal{G}$ does not admit invariant tensors of the type $d_{I}$,

$$
f_{I J}{ }^{K} d_{K}=0 .
$$

On the other hand we conclude, using appendix $\square$ again, that the functions $\beta_{I}$ can be assumed to transform under $\mathcal{G}$ according to the co-adjoint representation since this holds already for $s \beta_{I}$ :

$$
\delta_{I} \beta_{J}=f_{I J}^{K} \beta_{K}
$$

\footnotetext{
${ }^{9}$ If they were then the theory would be inconsistent since the equations of motion would imply " $5 \approx 0 "$.
} 
We now differentiate $(\mathbb{E} .11)$ with respect to $\hat{C}^{J}$. This yields, using $(\mathbb{E} .7)$ and (E.12),

$$
\hat{\partial}_{J} \hat{\partial}_{I} \beta=f_{J I}^{K} \beta_{K}-s\left(\hat{\partial}_{J} \beta_{I}\right) .
$$

Recall that $\hat{\partial}_{I}$ are derivatives with respect to anticommuting generators and that the structure constants $f_{I J}{ }^{K}$ are antisymmetric:

$$
\hat{\partial}_{I} \hat{\partial}_{J}=-\hat{\partial}_{J} \hat{\partial}_{I}, \quad f_{I J}{ }^{K}=-f_{J I}{ }^{K} .
$$

Hence, (E.13) implies $s \hat{\partial}_{(J} \beta_{I)}=0$. Due to $g h\left(\hat{\partial}_{J} \beta_{I}\right)=G-4<n-4$ we conclude, using lemma E.2 again:

$$
\hat{\partial}_{(I} \beta_{J)}=d_{I J} \delta_{G}^{4}+s \beta_{I J}, \quad d_{I J}=\text { const. }
$$

By means of arguments used already above we conclude that the $d_{I J}$ are the components of a symmetric invariant tensor of $\mathcal{G}$,

$$
d_{I J}=d_{J I}, \quad f_{I J}^{L} d_{L K}+f_{I K}{ }^{L} d_{J L}=0,
$$

and that the $\beta_{I J}$ can be assumed to be symmetric too and to transform according to their index picture under $\mathcal{G}$ :

$$
\beta_{I J}=\beta_{J I}, \quad \delta_{I} \beta_{J K}=f_{I J}^{L} \beta_{L K}+f_{I K}^{L} \beta_{J L} .
$$

Iterating this procedure one concludes the existence of a chain of functions $\beta_{I_{1} \ldots I_{m}}, m=0,1, \ldots$ satisfying

$$
\hat{\partial}_{\left(I_{1}\right.} \beta_{\left.I_{2} \ldots I_{m}\right)}=d_{I_{1} \ldots I_{m}} \delta_{G}^{2 m}+s \beta_{I_{1} \ldots I_{m}}
$$

where $d_{I_{1} \ldots I_{m}}$ are the components of invariant symmetric tensors of $\mathcal{G}$,

$$
\begin{aligned}
& d_{I_{1} \ldots I_{m}}=d_{\left(I_{1} \ldots I_{m}\right)}=\text { const. } \\
& \sum_{r=1}^{m}{f_{I I_{r}}}^{J} d_{I_{1} \ldots I_{r-1} J I_{r+1} \ldots I_{m}}=0
\end{aligned}
$$

and the $\beta_{I_{1} \ldots I_{m}}$ can be assumed to satisfy

$$
\begin{aligned}
& \beta_{I_{1} \ldots I_{m}}=\beta_{\left(I_{1} \ldots I_{m}\right)}, \quad g h\left(\beta_{I_{1} \ldots I_{m}}\right)=G-2 m-1, \\
& \delta_{I} \beta_{I_{1} \ldots I_{m}}=\sum_{r=1}^{m} f_{I I_{r}}{ }^{J} \beta_{I_{1} \ldots I_{r-1} J I_{r+1} \ldots I_{m}} .
\end{aligned}
$$


The occurrence of the constants $d_{I_{1} \ldots I_{m}}$ implies the presence of functions which are BRST exact in $\mathcal{A}_{2}$ but possibly not in $\mathcal{A}_{2,0}^{\text {inv }}$. These functions are invariant polynomials in the $\hat{F}^{I}$ :

$$
P_{2 m}(\hat{F})=d_{I_{1} \ldots I_{m}} \hat{F}^{I_{1}} \ldots \hat{F}^{I_{m}} .
$$

This follows directly from (E.4) and (E.16). To show this, we first decompose the functions $\beta_{I_{1} \ldots I_{m}}$ into parts with definite $\hat{C}$-degree:

$$
\beta_{I_{1} \ldots I_{m}}=\sum_{k \geq 0} \beta_{I_{1} \ldots I_{m}}^{k}, \quad \hat{C}^{I} \frac{\partial}{\partial \hat{C}^{I}} \beta^{k}{ }_{I_{1} \ldots I_{m}}=k \beta^{k}{ }_{I_{1} \ldots I_{m}} .
$$

Taking advantage of $(\overline{7.18})-(\sqrt{7.22})$, we conclude from (E.4)

$$
\alpha=\left(\delta+s_{1}\right) \beta^{0}+s_{2} \beta^{1}=s \beta^{0}+\hat{F}^{I} \hat{\partial}_{I} \beta^{1}
$$

since $\left(\delta+s_{1}\right) \beta^{0}+s_{2} \beta^{1}$ are the only contributions to $s \beta$ with vanishing $\hat{C}$ degree and since $\left(\delta+s_{1}\right) \beta^{0}=s \beta^{0}$ holds due to $\beta^{0} \in \mathcal{A}_{2,0}^{\text {inv }}$. The last term in $(\mathbb{E} .18)$ is now replaced by the following expression obtained from (E.11):

$$
\begin{aligned}
\hat{F}^{I} \hat{\partial}_{I} \beta^{1} & =\hat{F}^{I}\left(d_{I} \delta_{G}^{2}+\left(\delta+s_{1}\right) \beta^{0}{ }_{I}+s_{2} \beta^{1}{ }_{I}\right) \\
& =\delta_{G}^{2} P_{2}(\hat{F})+s\left(\hat{F}^{I} \beta^{0}{ }_{I}\right)+\hat{F}^{I} \hat{F}^{J} \hat{\partial}_{(J} \beta^{1}{ }_{I)}
\end{aligned}
$$

where we used $\left(\delta+s_{1}\right) \hat{F}^{I}=0, \hat{F}^{I} \beta^{0}{ }_{I} \in \mathcal{A}_{2,0}^{i n v}$ and $($ E.1 $)$, and $\hat{F}^{I} \hat{F}^{J} \hat{\partial}_{J} \beta^{1}{ }_{I}=$ $\hat{F}^{I} \hat{F}^{J} \hat{\partial}_{(J} \beta_{I)}^{1}$ (the latter holds since the $\hat{F}^{I}$ are commuting quantities). The last term in (E.19) is now replaced by an expression following from (E.15) and the procedure is iterated until the number of occurring $\hat{F}$ 's exceeds $n / 2$ (recall that monomials $\hat{F}^{I_{1}} \ldots \hat{F}^{I_{k}}$ vanish for $k>n / 2$ since $\hat{F}^{I}$ has $\hat{\xi}$-degree 2). This results finally in

$$
\alpha=\delta_{G}^{2 m} P_{2 m}(\hat{F})+s \hat{\beta}, \quad \hat{\beta} \in \mathcal{A}_{2,0}^{i n v}
$$

where

$$
\hat{\beta}=\sum_{k \leq n / 2} \hat{F}^{I_{1}} \ldots \hat{F}^{I_{k}} \beta_{{ }_{1} \ldots I_{k}}^{0} .
$$

We have thus proved that the invariant polynomials in the $\hat{F}^{I}$ are the only functions with ghost number $<n$ which are BRST exact in $\mathcal{A}_{2}$ but possibly not BRST exact in $\mathcal{A}_{2,0}^{\text {inv }}$. Together with Lemma E.2 this shows that any 
BRST invariant function in $\mathcal{A}_{2,0}^{i n v}$ with ghost number $G<n-1$ is BRST exact in $\mathcal{A}_{2,0}^{\text {inv }}$ up to an invariant polynomial in the $\hat{F}^{I}$ (including a constant for $G=0$ ) and a linear combination of those $q_{\alpha}^{*}$ which are in $\mathcal{A}_{2,0}^{i n v}$. The latter are just the $q_{\alpha^{0}}^{*}$ occurring in theorem 7.1 .

We still have to consider the cases $G=n-1$ and $G=n$. This is very easy. Namely any function $\alpha \in \mathcal{A}_{2,0}^{\text {inv }}$ with ghost number $n$ is necessarily BRST invariant since $n$ is the maximal ghost number a function in $\mathcal{A}_{2,0}^{i n v}$ can have. Furthermore, by definition of $\mathcal{A}_{2,0}^{\text {inv }}, \alpha$ has the form $\alpha=\hat{\Theta} L(\mathcal{T})$ with $\delta_{I} L(\mathcal{T})=0$ and $\hat{\Theta}$ as in $(7.8)$.

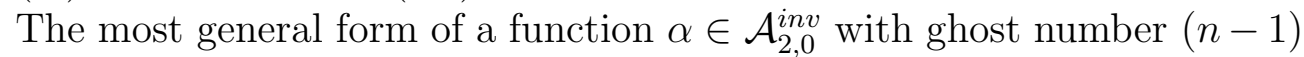
is $\alpha=X^{*}\left(\mathcal{T}, \mathcal{T}^{*}\right) \hat{\Theta}+J^{a}(\mathcal{T}) \partial \hat{\Theta} / \partial \hat{\xi}^{a}$ where $X^{*}\left(\mathcal{T}, \mathcal{T}^{*}\right)$ is $\delta_{I^{-}}$invariant and has antighost number 1 , and the $J^{a}$ satisfy (7.6). $s \alpha=0$ then requires $X^{*}$ and $J^{a}$ to solve (7.5) and therefore $X^{*}$ is a linear combination of the $X_{\Delta}^{*}$ up to a piece $\delta M\left(\mathcal{T}, \mathcal{T}^{*}\right)+D_{a} N^{a}\left(\mathcal{T}, \mathcal{T}^{*}\right)$, cf. subsection 7.1. This implies immediately that $\alpha$ is a linear combination of the $G_{\Delta}^{*}$ up to a piece which is $s$-exact in $\mathcal{A}_{2,0}^{i n v}$ (and given by $s\left[M \hat{\Theta}-(-)^{\varepsilon\left(N^{a}\right)} N^{a} \partial \hat{\Theta} / \partial \hat{\xi}^{a}\right]$ ) and a piece which does not depend on antifields at all. Since $\alpha$ has ghost number $(n-1)$, the latter is trivial in $\mathcal{A}_{2}$ by corollary 6.1 and therefore it is trivial in $\mathcal{A}_{2,0}^{\text {inv }}$ up to an invariant polynomial in the $\hat{F}^{I}$ by the above result. We conclude:

Lemma E.3 Any BRST invariant function $\alpha \in \mathcal{A}_{2,0}^{\text {inv }}$ is BRST exact in $\mathcal{A}_{2,0}^{\text {inv }}$ up to an invariant polynomial in the $\hat{F}^{I}$, a function of maximal $\hat{\xi}$-degree and a linear combination of the $G_{\Delta}^{*}$ and the $q_{\alpha^{0}}^{*}$,

$$
s \alpha=0, \alpha \in \mathcal{A}_{2,0}^{i n v} \Leftrightarrow \alpha=P(\hat{F})+\lambda^{\alpha^{0}} q_{\alpha^{0}}^{*}+\lambda^{\Delta} G_{\Delta}^{*}+\hat{\Theta} L(\mathcal{T})+s \beta
$$

where

$$
\delta_{I} P(\hat{F})=\delta_{I} L(\mathcal{T})=0, \quad \beta \in \mathcal{A}_{2,0}^{\text {inv }} .
$$

This result implies immediately part (i) of theorem 7.1 because of lemma E.1.

To prove part (ii) of the theorem we assume that $P\left(\hat{F}^{I}\right)$ is an invariant polynomial in the $\hat{F}^{I}$ of the form $(\mathbb{E} .17)$ and is BRST exact in $\mathcal{A}_{2,0}^{\text {inv }}$,

$$
P_{2 m}(\hat{F})=s \alpha, \quad \alpha \in \mathcal{A}_{2,0}^{i n v} .
$$

Now, from (7.3) and (7.4) we know that $P_{2 m}(\hat{F})$ can also be written as the BRST variation of a polynomial $\mathcal{P}$ in the $q_{(K)}$ and $f_{(K)}$,

$$
P_{2 m}(\hat{F})=s \mathcal{P}(q, f) .
$$


We have to prove that $P_{2 m}(\hat{F})$ vanishes for $2 m<n-1$. To that end we assume $P_{2 m}(\hat{F}) \neq 0$ and show that this leads to a contradiction for $2 m<$ $n-1$. First we note that $P_{2 m}(\hat{F}) \neq 0$ implies that the polynomial $\mathcal{P}(q, f)$ occurring in $(\mathrm{E} .23)$ has level $\leq 2 m-2$,

$$
\mathcal{P}(q, f)=\sum_{p \geq p_{0}} \mathcal{P}_{p}, \quad N_{\hat{\xi}} \mathcal{P}_{p}=p \mathcal{P}_{p}, \quad p_{0} \leq 2 m-2
$$

where $\mathcal{P}_{p_{0}} \neq 0$ is understood. Note that the level of a function in $\mathcal{A}_{2,0}^{\text {inv }}$ cannot be smaller than its ghost number. Since $\alpha$ has ghost number $(2 m-1)$ we know that it takes the form

$$
\alpha=\sum_{p \geq 2 m-1} \alpha_{p}, \quad N_{\hat{\xi}} \alpha_{p}=p \alpha_{p}
$$

From (E.23) and (E.22) we conclude

$$
s[\alpha-\mathcal{P}(q, f)]=0 .
$$

Consider now the case $2 m<n-1$. Then $\alpha-\mathcal{P}(q, f)$ has ghost number $2 m-1<n-2$ and therefore is BRST exact (in $\mathcal{A}_{2}$ ) according to lemma $\mathbb{E} .2$,

$$
2 m<n-1: \quad \alpha-\mathcal{P}(q, f)=s \beta .
$$

A constant cannot occur here because $\alpha-\mathcal{P}(q, f)$ has odd ghost number (given by $2 m-1$ ). (E.27) is now analyzed analogously to (7.40). Indeed, (E.27) contains a set of equations (7.42)-(7.45) to which $\alpha$ does not contribute since its level exceeds the level $p_{0}$ of $\mathcal{P}(q, f)$, cf. (E.24) and (E.25). Using the same arguments as in the discussion of (7.42)-(7.45), we conclude

$$
\beta=\hat{\beta}+\mathcal{Q}(q, f)+\mathcal{Q}^{\prime}(q, f)+\ldots
$$

where $\hat{\beta}$ satisfies

$$
\alpha-\hat{\mathcal{P}}(q, f)=s \hat{\beta}, \quad \ell(\hat{\beta}) \geq \hat{p}_{0}-1
$$

Here $\ell(\hat{\beta})$ and $\hat{p}_{0}$ are the levels of $\hat{\beta}$ and $\hat{\mathcal{P}}(q, f)$ respectively. The same arguments which we used to derive (7.47) lead now to the conclusion

$$
P_{\hat{p}_{0}}^{i}(\hat{F})=s_{1} \hat{\beta}_{\hat{p}_{0}-1}^{i}+\delta \hat{\beta}_{\hat{p}_{0}}^{i}, \quad \hat{\beta}_{\hat{p}_{0}-1}^{i}, \hat{\beta}_{\hat{p}_{0}}^{i} \in \mathcal{A}_{2,0}^{i n v} .
$$


Acting with $s_{1}$ on this equation results in $\delta\left(s_{1} \hat{\beta}_{\hat{p}_{0}}^{i}\right)=0$ due to $s_{1} P_{\hat{p}_{0}}^{i}(\hat{F})=0$ and (E.1). Using the acyclicity of $\delta$ in positive antighost number (appendix D), we conclude $s_{1} \hat{\beta}_{\hat{p}_{0}}^{i}+\delta \hat{\beta}_{\hat{p}_{0}+1}^{i}=0$ for some $\hat{\beta}_{\hat{p}_{0}+1}^{i} \in \mathcal{A}_{2,0}^{i n v}$. An iteration of the arguments (cf. proof of lemma E.1) implies the existence of $\hat{\beta}^{i}=\sum_{p \geq \hat{p}_{0}-1} \hat{\beta}_{p}^{i}$ such that

$$
P_{\hat{p}_{0}}^{i}(\hat{F})=s \hat{\beta}^{i}, \quad \hat{\beta}^{i} \in \mathcal{A}_{2,0}^{i n v} .
$$

This is an equation like $(\mathbb{E . 2 2})$, but for lower ghost number since we have $\hat{p}_{0}<2 \mathrm{~m}$. One can now repeat the arguments until the ghost number drops to zero. This provides the desired contradiction since a constant is not BRST exact.

Finally, consider (E.22) for $2 m=n-1$. We conclude from (E.26) by means of lemma E.2 that $\alpha-\mathcal{P}(q, f)$ is BRST exact up to a linear combination of the $q_{\alpha}^{*}$ :

$$
2 m=n-1: \quad \alpha-\mathcal{P}(q, f)=\lambda^{\alpha} q_{\alpha}^{*}+s \beta, \quad \beta \in \mathcal{A}_{2}, \quad \delta_{I} \beta=0 .
$$

Suppose that all $q_{\alpha}^{*}$ are in $\mathcal{A}_{2,0}^{\text {inv }}$, i.e. that $\left\{q_{\alpha}^{*}\right\}=\left\{q_{\alpha^{0}}^{*}\right\}$. Then (E.32) agrees with (E.27) if we replace there $\alpha$ by $\alpha^{\prime}=\alpha-\lambda^{\alpha} q_{\alpha}^{*}$. Hence, we can adopt the analysis following (E.27) even in the case $2 m=n-1$, and conclude $P_{n-1}(\hat{F})=0$ if $\left\{q_{\alpha}^{*}\right\}=\left\{q_{\alpha^{0}}^{*}\right\}$. This proves

Lemma E.4 No non-vanishing $\delta_{I}$-invariant polynomial in the $\hat{F}^{I}$ of ghost number $<n-1$ is BRST exact in $\mathcal{A}_{2,0}^{\text {inv }}$,

$$
d_{I_{1} \ldots I_{k}} \hat{F}^{I_{1}} \ldots \hat{F}^{I_{k}}=s \alpha, \alpha \in \mathcal{A}_{2,0}^{i n v}, 2 k<n-1 \quad \Leftrightarrow \quad d_{I_{1} \ldots I_{k}}=0 .
$$

(E.33) remains valid for $2 k=n-1$ if $\left\{q_{\alpha}^{*}\right\}=\left\{q_{\alpha^{0}}^{*}\right\}$.

Part (ii) of theorem 7.1 is equivalent to this lemma through lemma E.1.

\section{References}

[1] R.L. Bryant and P.A. Griffiths, Characteristic Cohomology of Differential Systems (I): General Theory, Duke University Mathematics Preprint Series, volume $1993 \mathrm{n}^{0} 1$ (January 1993).

[2] C.W. Misner and J.A. Wheeler, Ann. Phys. 2 (1957) 525. 
[3] W.G. Unruh, Gen. Relativ. Gravit. 2 (1971) 27.

[4] G. Barnich and M. Henneaux, Phys. Lett. B311 (1993) 123.

[5] R. Wald, Phys. Rev. D33 (1986) 3613.

[6] L. Alvarez-Gaumé and E. Witten, Nucl. Phys. B234 (1984) 269; W. Bardeen and B. Zumino, Nucl. Phys. B244 (1984) 421; F. Langouche, T. Schücker and R. Stora, Phys. Lett. B145 (1984) 342; L. Alvarez-Gaumé and P. Ginsparg, Ann. Phys. 161 (1985) 423; L. Bonora, P. Pasti and M. Tonin, J. Math. Phys. 27 (1986) 2259; T. Schücker, Commun. Math. Phys. 109 (1987) 167.

[7] F. Brandt, N. Dragon and M. Kreuzer, Nucl. Phys. B340 (1990) 187.

[8] C. Becchi, A. Rouet and R. Stora, Commun. Math. Phys. 42 (1975) 127; Ann. Phys. 98 (1976) 287; I.V. Tyutin, Gauge invariance in field theory and statistical mechanics, Lebedev preprint FIAN, ${ }^{0} 39$ (1975).

[9] J. Zinn-Justin, Renormalisation of gauge theories in Trends in elementary particle theory, Lecture notes in Physics $\mathrm{n}^{0} 37$, Springer (Berlin: 1975).

[10] R.E. Kallosh, Nucl. Phys. B141 (1978) 141.

[11] B. de Wit and J.W. van Holten, Phys. Lett. B79 (1978) 389.

[12] I.A. Batalin and G.A. Vilkovisky, Phys. Lett. B102 (1981) 27; Phys. Rev. D28 (1983) 2567; Phys. Rev. D30 (1984) 508.

[13] J. Wess and B. Zumino, Phys. Lett. B37 (1971) 95.

[14] G. Barnich, F. Brandt and M. Henneaux, Local BRST cohomology in the antifield formalism: I. General theorems, preprint ULB-TH-94/06, NIKHEF-H 94-13, hep-th/9405109, to appear in Commun. Math. Phys.

[15] G. Barnich, F. Brandt and M. Henneaux, Phys. Rev. D51 (1995) R1435.

[16] D. Finkelstein and C.W. Misner, Ann. Phys. 6 (1959) 230.

[17] E. Noether, Nachr. v. d. Kgl. Ges. d. Wiss. z. Göttingen, Math.-phys. Kl., 2 (1918) 235. 
[18] C.G. Torre and I.M. Anderson, Phys. Rev. Lett 70 (1993) 3525.

[19] F. Brandt, Structure of BRS-invariant local functionals, preprint NIKHEF-H 93-21, hep-th/9310123.

[20] J.M.L. Fisch and M. Henneaux, Commun. Math. Phys. 128 (1990) 627;

M. Henneaux, Nucl. Phys. B (Proc. Suppl.) 18A (1990) 47.

[21] O. Moritsch and M. Schweda, Helv. Phys. Acta 67 (1994) 289.

[22] F. Takens, J. Differential Geometry 14 (1979) 543; I.M. Anderson and T. Duchamp, Amer. J. Math. 102 (1980) 781.

[23] I.M. Anderson, Contemp. Math. 132 (1992) 51, The variational bicomplex, Academic Press (Boston: 1994).

[24] A.M. Vinogradov, Sov. Math. Dokl. 18 (1977) 1200, 19 (1978) 144, 19 (1978) 1220; M. De Wilde, Lett. Math. Phys. 5 (1981) 351; W.M. Tulczyjew, Lecture Notes in Math. 836 (1980) 22; P. Dedecker and W.M. Tulczyjew, Lecture Notes in Math. 836 (1980) 498; T. Tsujishita, Osaka J.of Math. 19 (1982) 311; L. Bonora and P. Cotta-Ramusino, Commun. Math. Phys. 87 (1983) 589; P.J. Olver, Applications of Lie Groups to Differential Equations, Graduate Texts in Mathematics, volume 107, Springer Verlag (New York: 1986); R.M. Wald, J. Math. Phys. 31 (1990) 2378; L.A. Dickey, Contemp. Math. 132 (1992) 307.

[25] F. Brandt, N. Dragon and M. Kreuzer, Nucl. Phys. B332 (1990) 224; M. Dubois-Violette, M. Henneaux, M. Talon and C.M. Viallet, Phys. Lett. B267 (1991) 81.

[26] C.G. Torre, Some remarks on gravitational analogs of magnetic charge, preprint, gr-qc/9411014.

[27] R. Stora, Continuum Gauge Theories in New Developments in Quantum Field Theory and Statistical Mechanics, eds. M. Lévy and P. Mitter (Plenum: 1977), Algebraic Structure and Topological Origin of Anomalies in Progress in Gauge Field Theory, eds. G. 't Hooft et al. (Plenum: 1984); B. Zumino, Chiral Anomalies and Differential Geometry in Relativity, Groups and Topology II, eds. B.S. De Witt and R. Stora (NorthHolland: 1984); L. Baulieu, Phys. Rep. 129 (1985) 1. 
[28] S.P. Sorella, Commun. Math. Phys. 157 (1993) 231.

[29] L. Baulieu and M. Bellon, Nucl. Phys. B266 (1986) 75; L. Baulieu, M. Bellon and R. Grimm, Nucl. Phys. B294 (1987) 279; F. Brandt, Class. Quantum Grav. 11 (1994) 849.

[30] L. O'Raifeartaigh, Group structure of gauge theories, Cambridge University Press (Cambridge: 1986), section 5.6.

[31] M. Dubois-Violette, M. Talon and C.M. Viallet, Commun. Math. Phys. 102 (1985) 105.

[32] F. Brandt, N. Dragon and M. Kreuzer, Nucl. Phys. B332 (1990) 250.

[33] P. Gilkey, Adv. in Math. 28 (1978) 1.

[34] G. Barnich, F. Brandt and M. Henneaux, Local BRST cohomology in the antifield formalism: II. Application to Yang-Mills theory, preprint ULBTH-94/07, NIKHEF-H 94-15, hep-th/9405194, to appear in Commun. Math. Phys..

[35] M. Henneaux, Commun. Math. Phys. 140 (1991) 1.

[36] G. Barnich, F. Brandt and M. Henneaux, Phys. Lett. B346 (1995) 81.

[37] J.L. Koszul, Bull. Soc. Math. France 78 (1950) 65; C. Chevalley and S. Eilenberg, Trans. Am. Math. Soc. 63 (1953) 589; G. Hochschild and J.P. Serre, Ann. Math. 57 (1953) 591.

[38] M. Henneaux and C. Teitelboim, Quantization of Gauge Systems, Princeton University Press (Princeton: 1992). 